\title{
BNL 50718
}

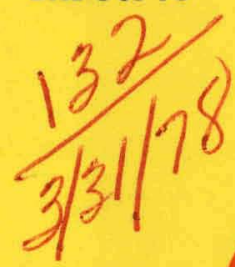

Hh. 1962

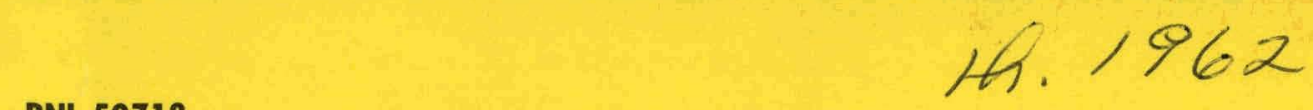

$\frac{13}{3 / 31 / 178}$
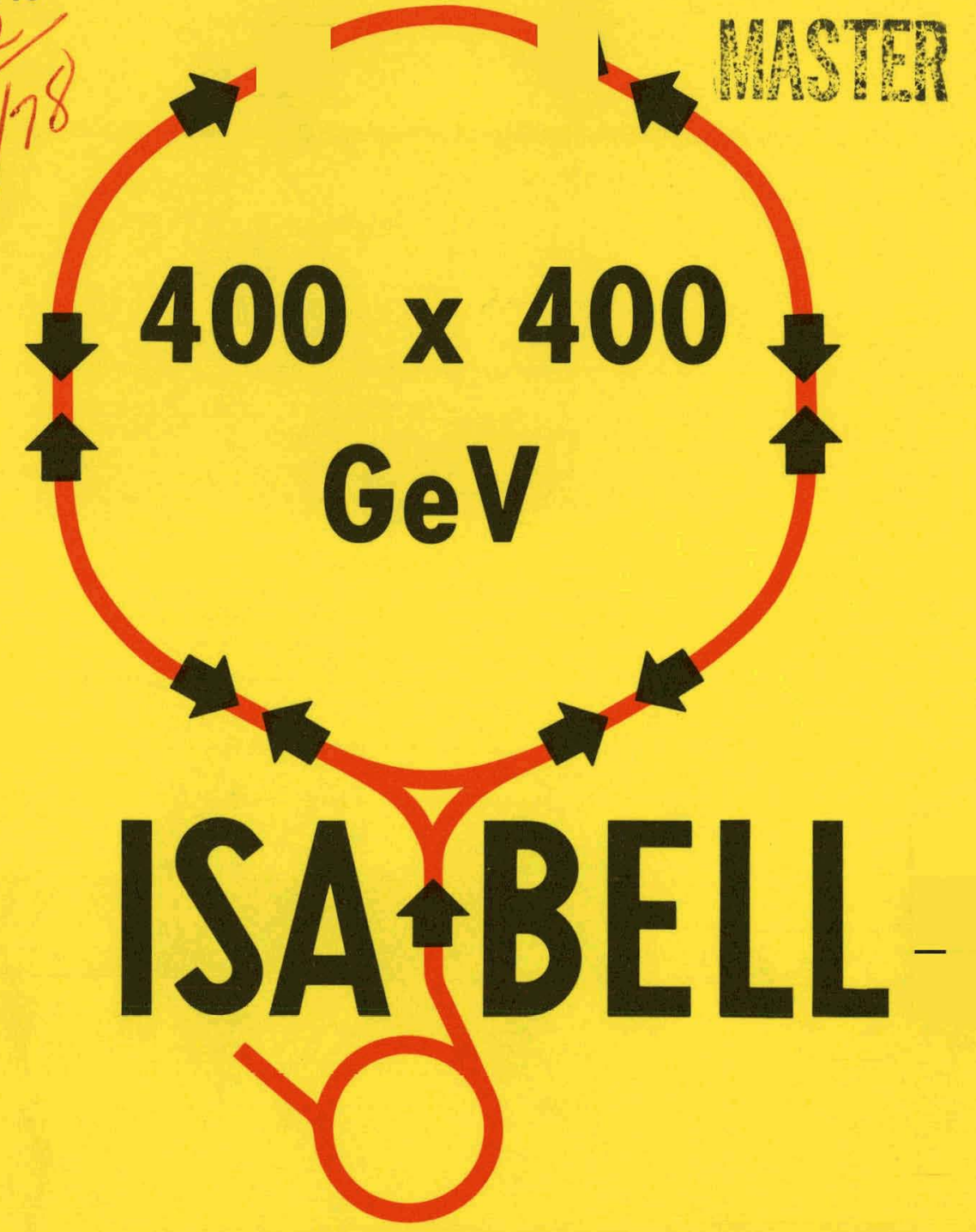

\section{A PROTON-PROTON}

\section{COLLIDING BEAM FACILITY}

\section{BROOKHAVEN NATIONAL LABORATORY} UPTON, NEW YORK 11973 


\section{DISCLAIMER}

This report was prepared as an account of work sponsored by an agency of the United States Government. Neither the United States Government nor any agency Thereof, nor any of their employees, makes any warranty, express or implied, or assumes any legal liability or responsibility for the accuracy, completeness, or usefulness of any information, apparatus, product, or process disclosed, or represents that its use would not infringe privately owned rights. Reference herein to any specific commercial product, process, or service by trade name, trademark, manufacturer, or otherwise does not necessarily constitute or imply its endorsement, recommendation, or favoring by the United States Government or any agency thereof. The views and opinions of authors expressed herein do not necessarily state or reflect those of the United States Government or any agency thereof. 


\section{DISCLAIMER}

Portions of this document may be illegible in electronic image products. Images are produced from the best available original document. 


$$
\begin{gathered}
\text { BilL } 50718 \\
\text { (Particle Accelerators and } \\
\text { High-Voltage Machines - TID 4500) }
\end{gathered}
$$$$
\text { I S A B ELLE }
$$

A $400 \times 400$ GEV PROTON-PROTON COLLIDING BEAM FACILITY

DISTRIBUTION OF THIS DOCUMENT IS UNLIMITED

BROOKHAVEN NATIONALLABORATORY)

A S S O C I A T E D.UN I VER S I T I E S, I $N$ C; UNDER CONTRACT NO. EY-76-C-02-0016 WITH THE United States Department of Energy 


\section{N U I I C E}

This report was prepared as an account of work sponsored by the United States Government. Neither the U.S. nor the U.S.

Department of Energy, nor any of their employees, nor any of their contractors, subcontractors, or their employees, makes any warranty, express or implied, or assumes any legal liability or responsibility for the accuracy, completeness or usefulness of any information, apparatus, product or process disclosed, or represents that its use would not infringe privately owned rights.

PRINTED IN THE UNITED STATES OF AMERICA

$$
\text { Available from }
$$

National Technical Information Service

U.S. Department of Commerce

$$
5285 \text { Port Royal Road }
$$

Springfield, VA 22161

Price: Printed Copy $\$+00 ;$ Microfiche $\$ 3.00$ 


\section{PREFACE}

This is the conceptual design report for the construction of an Intersecting Storage Accelerator, ISABELLE, to be located at Brookhaven National Laboratory. At this major research facility beams of protons with energies up to $400 \mathrm{GeV}$ will be collided in six experimental areas. At each area particle physicists will install detector apparatus to study the interaction and reaction products for such very high energy collisions. The proposal results from several years of study and development work on such a facility. The encouragement and sustained support of the Department of Energy previously ERDA) is gratefully acknowledged.

Individuals from the Accelerator and Physics departments of Brookhaven have done the detailed work involved in preparing this document. Their work has been made easier by the results of numerous Workshops in which future university users of the facility participated. Special recognition should be given to the efforts of $\mathrm{H}$. Hahn, who headed the ISABELLE Division during the past few years. Preliminary conceptual design work was organized by M. Barton with physics motivation from the Physics Department, chaired by N. Samios.

The work was organized through three efforts; a Parameter Committee chaired by H. Hahn, a Finance Committee chaired by J. Spiro, and an Editorial Committee chaired by J. Sanford. The committee members, whose names are listed below have made major contributions to this proposal, and their help is acknowledged with thanks.

Members of the Parameters Committee: M.Q. Barton, D.P. Brown, E.D. Courant, H.W.J. Foelsche, S.T. Giordano, H. Hahn, H.J. Halama, D.A. Kassner, A.D. McInturff, M. Month, F. Pedersen, G. Parzen, A.M. Thorndike. A. van Steenbergen participated in earlier stages of Committee deliberations.

Members of the Finance Committee: S.T. Giordano, H. Hahn, D.A. Kassner, H. McChesney, Jr., P.V. Mohn, I.J. Polk, W.B. Sampson, R.P. Shutt, J. Spiro.

Members of the Editorial Committee: P.F. Dahl, H.W.J. Foelsche, - Hahn, J.C. E rrera, T.F. Kycia, F. Palge, R.F. Peierls, E.C. Raka, .R. Sanford, A.M. Thornd1ke.

The manuscript was typed by J. Ferrero and A. Rementer with the organization of material by $P$. Hughes. The drawings were under the supervision of $\mathrm{P}$. Dah1 and J. Herrera. The report was printed by the capable staff of Graphics Arts under the supervision of R. Brown. All of these individuals have played decisive roles in preparing this proposal.

James R. Sanford January 1, 1978 


\section{THIS PAGE}

\section{WAS INTENTIONALLY}

LEFT BLANK 


\section{TABLE OF CONTENTS}

I. INTRODUCTION AND SUMMARY OF THE PROPOSAL 1

Summary Description of the ISABELLE Proposal 4

II. PHYSICS AT ISABELLE

1. Introduction 9

2. Physics Objectives 15

3. Typical Experiments and Detectors 51

III. DESCRIPTION OF ISABELLE

1. Overview of ISABELLE Facility 74

2. Magnet Ring Structure and Lattice $\begin{array}{ll}\text { Characteristics } & 81\end{array}$

3. Performance 90

4. Beam Transfer, Stacking, and Acceleration 95

5. Magnet System 110

6. Refrigeration System 123

7. Vacuum System 130

8. Power Supplies, Instrumentation, and

9. Physical Plant and Experimental Halls 145

10. Operation and Safety 156

IV. COST ESTIMATE AND SCHEDULE

1. Cost Estimate 158

2. Critical Milestones and Schedule 159

3. Anticipated Equipment and Operating Needs 165 APPENDIX

ISABELLE Parameter List

REFERENCES 


\section{INTRODUCTION AND SUMMARY \\ OF THE ISABELLE PROPOSAL}

ISABELLE will provide a research instrument capable of studying proton-proton collisions to very high energies. One ring of magnets carrying protons will be interlaced with a second ring of magnets carrying protons circulating in the opposite sense. The beams will cross at six locations where early head-on collisions take place. Research halls will be - onstructed at these regions for the housing of the particle detectors used in studying the proton-proton collisions.

The advantage of using colliding beams to achieve high center-of-mass energies was recognized many years ago. Following the operation of the $30 \times 30 \mathrm{GeV}$ proton-proton ISR at CERN in 1971, proposals were made for the extension in energy and intensity of such devices. Electron-positron storage rings are currently in operation with major increases in energy planned for about 1980. Figure I.I shows the energy available from both fixed target proton synchrotrons and storage rings. As can be seen, ISABELLE would enjoy a commanding position.

The construction of high-energy proton-proton storage rings at Brookhaven has been studied extensively.* Proposals were prepared leading up to a large summer study in 1975, and workshops in 1976 and 1977. These meetings were held for the purpose of exploring the design features and intended use of ISABELLE. They helped to clarify the important features of the oject, and to enlist the support of the university research - smmunity.

* See the 1976 proposal for a history of the development of ISABELLE including references to earlier work. 


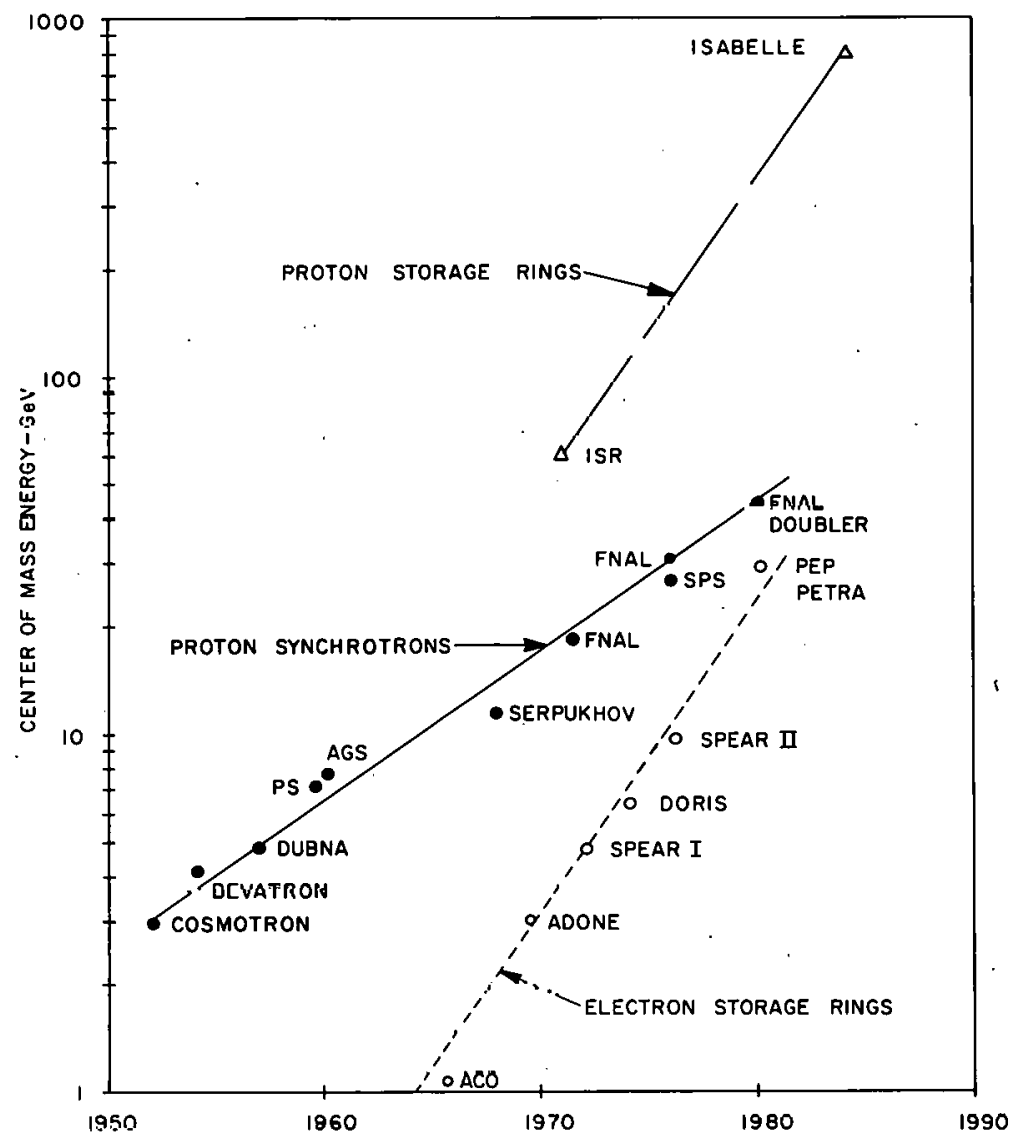

Fig. I.1. Growth of the center-of-mass energy for high energy accelerators and storage rings over recent decades. 
The Department of Energy (DOE) has provided increased accelerator R\&D funds for ISABELLE since FY 1975. This has been supplemented by Construction, Planning and Design (CP\&D) funds first awarded in March 1976. These funds have been used to eva1uate the cost and design problems associated with building such a research facility. In the course of events it was found desirable to raise the energy to $400 \mathrm{GeV}$ in each beam and to redesign the magnet configuration for a six-fold geometry.

In parallel with these developments, the high-energy community, through subpanels of HEPAP, considered ISABELLE and other national projects. These panels recommended increased financial aid for the supporting $R \& D$ activities, and subsequent authorization of the project. They saw this project as part of a new national initiative in high-energy physics that would bring a major research facility to Brookhaven while extending the usefulness of the existing AGS. The most recent panel (HEPAP 1977) met in June, 1977 and sustained the commitment to ISABELLE. Their unanimous opinion is : "We recommend as our first priority the authorization of the BNL proposal for the construction of the proton-proton colliding beam facility ISABELLE to operate with a maximum energy of about 400 $\mathrm{GeV}$ per beam and a peak luminosity of $10^{33} \mathrm{~cm}^{-2} \mathrm{sec}^{-1}$." This document describes the proposal that was made to the panel and to DOE.

The proposal is divided into several major parts. Following this introduction is an overall summary of the proposal covering its highlights. Part II contains a thorough discussion of the physics objectives that can be addressed by the storage ring. $t$ begins with an explanation of some theoretical concepts and uestions which are of great interest to high energy physicists. Then follows a brief discussion of possible experiments that might be assembled at the interaction regions to test these concepts. 
The third part of the proposal goes into the details of the design of the intersecting storage accelerators. It begins with a description of the entire facility and the design of the magnet ring structure. The processes of proton beam accumulation and acceleration are thoroughly described. The discussion then turns to the design of the components and subsystems for the accelerator. The accelerator elements are described followed by a description of the physical plant. In the latter case, the project has already benefited from the help of Ammann \& Whitney, the architect/engineering firmselected to design the conventional construction.

The cost estimate and time scales are displayed in Part IV. Here the estimate has been based on the experience gained from. working with the prototype units at the laboratory. The availability of Construction, Planning and Design (CP\&D) funds has allowed the unit cost estimates to be examined by competent consultants and industrial firms.

\section{SUMMARY DESCRIPTION OF THE ISABELLE PROPOSAL}

TSABELLE would p̈róvide two interlaced storage $r$ ing proton accelerators with counter-rotating beams, each with an energy up to $400 \mathrm{GeV}$ intersecting in six experimental areas. At the top energy of $400 \mathrm{GeV}$ in each circulating beam, $800 \mathrm{GeV}$ of energy will be available from each proton-proton collision. The most significant aspect of ISABELLE is the high "center-of-mass" energy. For example, to provide an equal center-of-mass energy when a moving proton strikes a proton at rest, the moving proton would need an energy of $340,000 \mathrm{GeV}$. The only existing protonproton colliding beam machine is at CERN in Switzerland where $30 \mathrm{GeV}$ proton beams collide, giving a center-of-mass energy of $60 \mathrm{GeV}$. ISABELLE can operate at this level and extend more than ten times higher in energy than the ISR. 
The second important parameter of ISABELIE is the intensity of the beams. In colliding beam machines, the interaction rate is the product of the cross section and the luminosity, $R=\sigma L$. Luminosity, expressed in units of centimeter ${ }^{-2}$ second $^{-1}$, is a function only of the parameters of the machine, while the cross section is related to the particular interaction being studied and is in units of centimeter ${ }^{2}$. ISABELLE would provide a luminosity of up to $10^{33} \mathrm{~cm}^{-2} \mathrm{sec}$, a factor 20 larger than the uminosity now available at the ISR. For the production of the -nusual particles that are expected to exist at these high energies, this high luminosity is essential.

ISABELLE will provide a.flexible facility which can investigate a wide range of questions of current interest in particle physics. The large increase in center-of-mass energy should provide useful new information on strong interactions: typical measures of these interactions like the total cross section have been found to be increasing with energy at the ISR and FNAL. Furthermore, ISABELIE will greatly extend the study of the production of hadrons and leptons at large transverse momentum. Experiments at the ISR and at FNAL have found this production to be much more copious than was expected. While these results are not yet understood, it is believed that they are related to the existence of smaller constituents within the proton. Perhaps the single most important area of research would be in the weak interactions where it should be possible to observe the production of the intermediate vector bosons, $W^{ \pm}$and $z^{\circ}$ iich have been hypothesized for many years as the mediator of se weak interaction. Their masses are predicted to be about 60-80 GeV/ $/ \mathrm{c}^{2}$, well within the range of ISABELLE.

The storage rings would be located in the Northwest corner of the Brookhaven National Laboratory site (Fig. I.2). The 


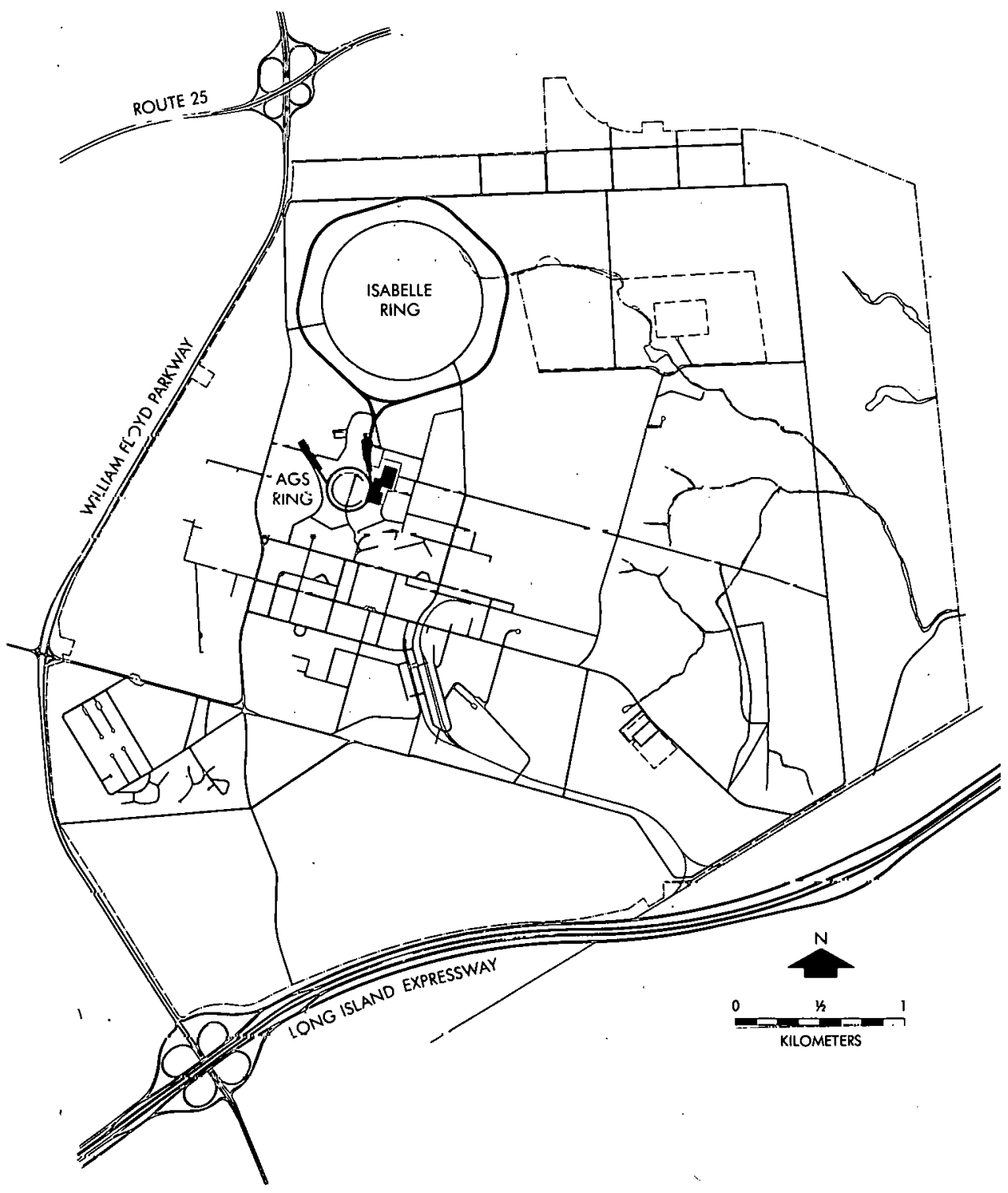

Fig. I.2. Brookhaven National Laboratory site map showing the location of the AGS and ISABELLE. 
protons at $30 \mathrm{GeV}$ would be provided to the ISABELLE rings from the existing Alternating Gradient Synchrotron (AGS). About three hundred pulses would be stacked in each ring building up to a current of 8 ampere. Following that, over a period of about four minutes, each beam of protons would be accelerated to whatever energy the experimenters wished up to $400 \mathrm{GeV}$. Asymmetric energies would be available, for example, $400 \mathrm{GeV}$ in one beam and $200 \mathrm{GeV}$ in the other beam. There will be six regions in hich the proton beams interact. At the intersecting points the beams would have a vertical height of about $\frac{1}{2}$ mand would interact over a length of about $50 \mathrm{~cm}$ crossing at an angle of about $0.5^{\circ}$. Experimental apparatus will surround the interaction region and in turn be covered by an experimental ha11. Some 8 to 12 experiments might be simultaneously set up in the six areas.

The most important new feature of ISABELLE is the use of superconducting magnets in the two rings. The superconducting excitation coils are made from NbTi braided wires operating at $3.8 \mathrm{~K}$. The bending magnets or dipoles are $4-3 / 4 \mathrm{~m}$ in length and operate at $50 \mathrm{kG}$. Full-size prototypes have operated at this magnetic field and with a field quality meeting the design goals. The focusing magnets or quadrupoles are about $1-2 / 3 \mathrm{~m}$ in length. The first quadrupole has been tested satisfactorily. In total, nearly 1100 superconducting magnets would make up the two rings. The high magnetic field relative to that produced by copper-iron magnets reduces the circumference for a given energy and thus he cost. In this case, the circumference is $3.8 \mathrm{~km}$ and the wo magnet rings are housed in a single tunnel some $4.6 \mathrm{~m}$ in diameter completely shielded with earth. The high magnetic field also improves the operating characteristics of the machine and more importantly, the superconducting nature of the magnets reduces the need for electrical power and consequently the 
operating cost. For example, the electrical energy used by superconducting magnets is about $25 \%$ that used by conventional magnets made of copper and iron.

The protons circulate through a vacuum tube $8.8 \mathrm{~cm}$ in diameter within the magnets. This vacuum tube will be at room temperature and insulated from the Inner wall of the dewar system which contains the superconducting magnet coil. The high vacuum technology required is extremely demanding as the beam tube must operate at about $3 \times 10^{-11}$ Torr.

In addition, there will be many other accelerator technologies involved in the construction of ISABELLE, such as radio-frequency accelerating systems, high current power supplies, large cryogenic and refrigeration systems, safety and radiation protective circuits, and overall control by means of a computer system using sophisticated instrumentation.

Particle detectors for high-energy physics experiments will be installed at every intersection region. The apparatus will have been constructed and tested in the experimental area by the experimenters during times when the storage rings are turned off. During operation many groups of researchers will take data simultaneously. ISABELLE will be a national facility where experiments are done by qualified researchers from throughout the world. Physicists from Brookhaven will operate the facility and do experiments, although the majority of experiments will be done by physicists and students from university research groups within the United States.

The cost is estimated at $\$ 168,350,000$ with an additional sum of $\$ 27,830,000$ for contingency and $\$ 43,570,000$ for inflationary factors occurring during the five-year construction period. Alternative funding plans are currently being considered by the U. S. Department of Energy. 


\section{PHYSICS AT ISABELLE}

\section{INTRODUCTION}

With its large increase in center-of-mass energy ${ }^{*}$ over currently available values and its high luminosity, ISABELLE will permit studies of the interaction of matter at smaller distances than ever before. What can we expect to learn? The most exciting prospect is to discover something quite unexpected which will give us iome new insight into the structure of matter, while the most cerLain benefit is the opportunity to extend current ideas and models to significantly higher energies. These contrasting possibilities are illustrated by the simplest measurement, the total protonproton cross section, as shown in Fig. II.1. The rise observed at the highest measured energies lends itself to various reasonable extrapolations which can only be resolved by measurements at higher energies, but at the same time we note that similar "reasonable" extrapolations at $1 \mathrm{GeV}$ would have quite missed the sudden unexpected turnover.

Of particular interest are those investigations which will serve to test and explore a general picture of particles and their interactions which has been gaining increasing acceptance recently (Glashow 1975, Nambu 1976). This picture was itself stimulated by "unexpected" experimental discoveries of the past few years at presently available machines, particularly following the discovery of the J/ $\Psi$ particle at the Brookhaven AGS and SLAC. According to this picture, the proton and other hadrons are made up of pointike spin one-half constituents whose weak and electromagnetic ateractions resemble those of the leptons. Their strong interactions are such that only aggregates of such constituents can exist as stable or metastable systems, and thus the observable hadrons are extended structures. To date, the pointlike nature of

\footnotetext{
*For future reference the square of the total center-of-mass energy is denoted by "s," components of vectors along the beam direction by the subscript " $\|$," and transverse components by the subscript "1."
} 


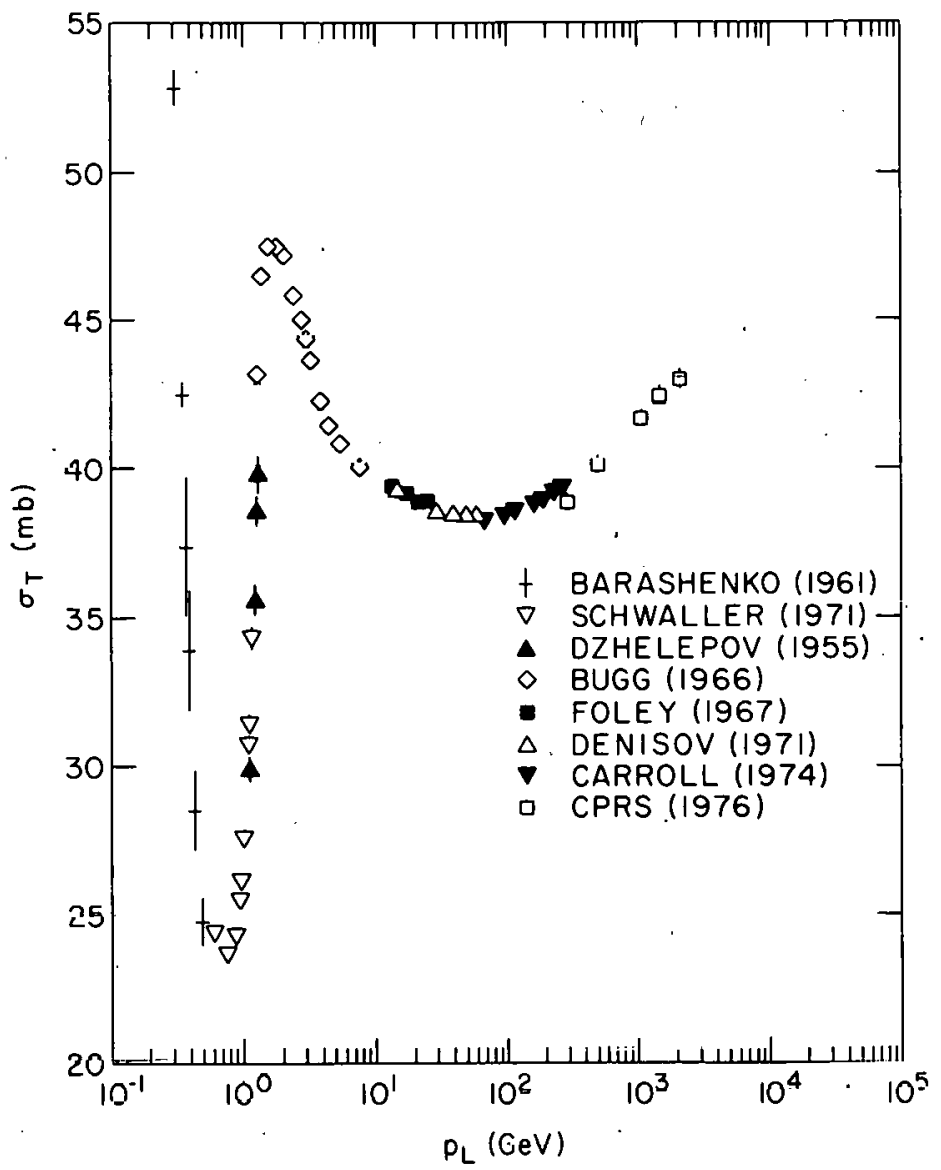

Fig. II.1. Total proton-proton cross section as a function of incident proton momentum. 
the constituents has been suggested by the results of deep. inelastic leṕton scattering. These constituents are generally referred to as "quarks" or "partons," although these terms initially were introduced to cover particular specific models.

This general picture of the proton as an extended composite of point-like constituents leads us to seek three kinds of experimental information. First of all we can make use of the proton as a source of quarks and study the properties of colliding quarks $r$ annihilating quark-antiquark pairs, leading to the production. of the W-mesons and charmed particles.

Secondly, we can study the results of quark-antiquark annihilation into a heavy photon, decaying into lepton pairs. The annihilation and decay is a we11. understood quantum electrodynamic process. These observations can then be used to draw conclusions about the distribution of quarks in the proton.

Thirdly, there are experiments dealing with processes in which the quarks in a single hadron act collectively, but in which the transfer of momentum may change the configuration of the quarks within the hadrons. This accounts for the bulk of the hadronic total cross section. The main interest of ISABELLE here is the opportunity to extend present dynamical ideas to a greatly increased energy range.

The study of massive lepton pair production is crucial to developing confidence in this whole picture as described. While such study is not likely to demonstrate rigorously the presence E point-1ike constituents, this process can provide strong suprrting evidence. The key to this is the observation of "scaling". Scaling is the assertion that, for sufficiently high energies, the cross section for production of such a virtual heavy photon should depend only on the ratio of its mass to the total collision energy. This follows from the quark model, (providing transverse motion is neglected or assumed limited), but it will also be true of any process in which the photon is formed from two objects whose four- 
momenta are limited. Present data (Lederman 1977) show no gross departures from scaling through ISR energies.

The test of the model lies in combining scaling with the requirement that the longitudinal motion of the lepton pairs should be consistent with that predicted by the model, using quark distributions measured in deep inelastic electron scattering. It is a consistency test and although it appears to be satisfied at presently available energies (see Fig. II.2 and the discussion below) it will require the extension to ISABELLE to provide a significant test of the agreement.

The quark picture allows reasonably quantitative estimates of the production of the intermediate vector bosons responsible for the weak interactions. The search for these $W$ particles has always been high on the 1 ist of objectives for ISABELLE (ISA 1972-76). Almost any unified model of weak and electromagnetic interactions leads to the prediction of masses for these objects of $50-100 \mathrm{GeV} / \mathrm{c}^{2}$; well within the range of observation of ISABELLE. With these more quantitative models, however, we are now in a position where even a null resilt would be informative.

In addition to providing reassurance that the estimates of $W$ production are plausible, the study of guark interactions themselves is of considerable interest. The production cross sections for processes other than weak and electromagnetic annihilation are mish less certain, but the potential insights into the nature and interastion of hadronic constituents are mich greater.

So far, we have discussed the expactatioas for what we might learn at ISABELLE. How well can these crucial phenonena be maasured if they do take place? Since the inception of the ISABELLE proposal, extensive studies have been made to discuss hypothetical experiments. W1th reasonably conservative extrapolation of existing techniques, the experimental outlook is encouraging. In the remainder of this section we discuss these points in some- 


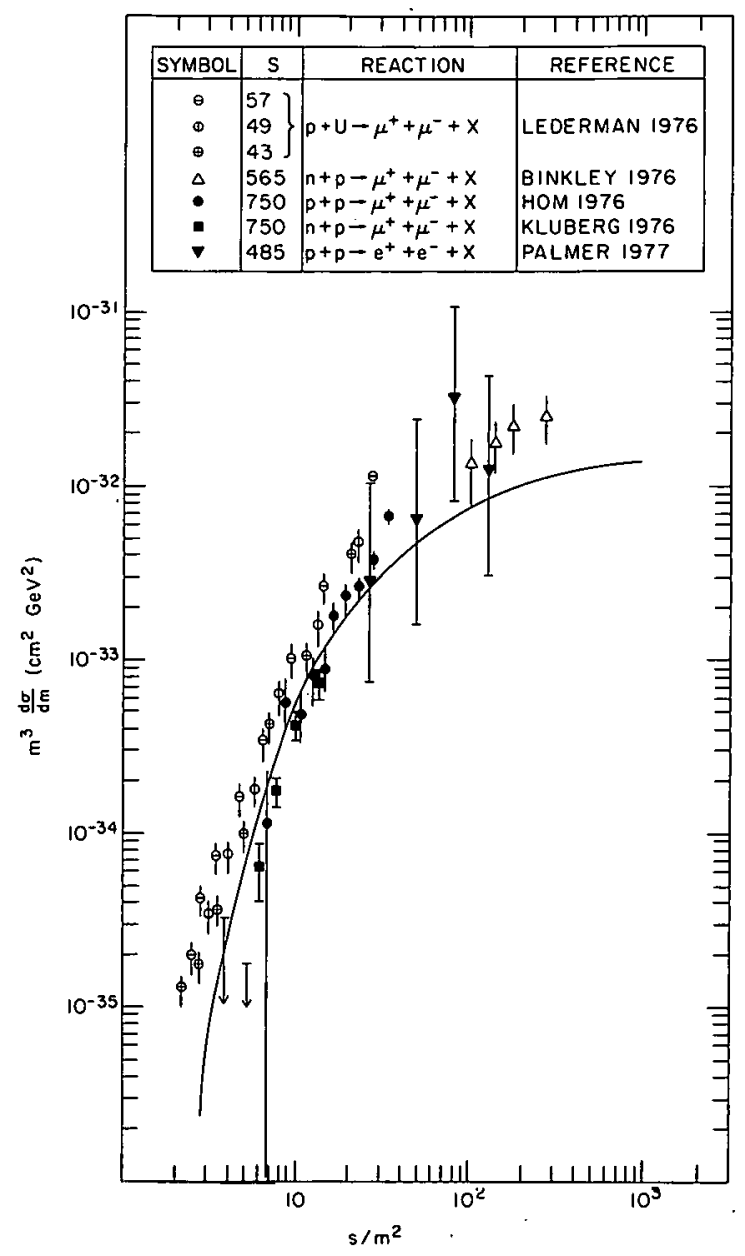

Fig. II.2. Evidence for scaling in lepton pair production. The "experimental" points shown have been obtained from actual data by using a quarkparton model to extrapolate into unobserved regions of longitudinal momentum. The model, including color SU(3), is that described in the text. The solid curve is the absolute prediction of the model. 
what more detail, including a description of equipment that could be used to investigate some of the questions of greatest interest. 


\section{Production of $W$ Mesons}

The most direct way to test the underlying theory of weak interactions is to search for the $W$-mesons, the weak vector bosons whose coupling to fermions is assumed to be responsible for the weak force (Ge11-Mann 1959). Their masses must be large, as is clear from the fact that low energy weak interactions can be sucsssfully described in terms of a local four-fermion interaction. ley must exist in both charged and neutral types: that is clear from the observation of neutral weak currents (Williams 1976).

Even if we had no idea of what mass range to expect, the opening up of a new kinematic range at ISABELLE, with an increase in $s$ of more than two orders of magnitude, would make the search for the $W$ an important objective. In fact, however, current theoretical ideas point very strongly to the $W$ mass being in a range particularly suitable to ISABELLE experiments.

The most important ingredient of these ideas is that the weak and electromagnetic interactions are closely related and that the $W$ mesons are massive analogs of the photon, carrying the same intrinsic coupling strength. This immediately leads to the mass estimate

$$
\mathrm{m}_{\mathrm{W}} \approx\left(\mathrm{e}^{2} / \mathrm{G}_{\mathrm{F}}\right)^{\frac{1}{2}} \approx 100 \mathrm{GeV} / \mathrm{c}^{2}
$$

where $e$ is the electric charge and $G_{F}$ the Fermi constant.

More specific theories allow detailed predictions (Abers 1973). : an example, the Weinberg-Salam mode1 [the prototype of a class : nonabelian gauge models of unified weak and electromagnetic interactions (Weinberg 1967, Salam 1967)] predicts the masses in terms of $e, G_{F}$, and one other parameter ${ }_{W}$ (specifying the weak isoscalar/isovector mixing of the neutral current). With an estimate of $\theta_{W}$ from observations of neutrino reactions, the model gives

$$
\mathrm{m}_{W}+\approx 60 \mathrm{GeV} / \mathrm{c}^{2}, \mathrm{~m}_{\mathrm{W}} \mathrm{0} \approx 77 \mathrm{GeV} / \mathrm{c}^{2} \text {. }
$$


More general models of this class (Barnett 1976) in general predict the existence of larger numbers of $W$ mesons. In almost all cases some will have masses less than or equal to those predicted by the Weinberg-Salam model. It is therefore evident that even the absence of a $\mathrm{W}$ meson in this mass range would be of considerable theoretical importance, while its discovery would obviously be a triumph for both theory and experiment.

To draw conclusions from any experiment it is necessary to have some estimate of the cross sections for producing $W$ mesons of this mass range at ISABELLE energies. To make such predictions, one can make use of the hypothesis of CVC [conserved vector current (Feynman 1958)] which is more general than the specific unified weak and electromagnetic models. According to CVC the total cross section for production of a $W$ meson of mass $\mathrm{m}_{W}$ is related to the cross section for production of a lepton pair of the same mass:

$$
\left.\sigma_{W} \gg 0.1 \mathrm{M}^{3} \frac{\mathrm{d} \sigma}{\mathrm{dM}}\right|_{\mathrm{M}=\mathrm{m}_{\mathrm{W}}}
$$

where $d \sigma / d M$ is the cross section for the process

$$
p+p \rightarrow l+l+x
$$

and $M$ is the mass of the dilepton pair. (Yamaguchi 1966, Palmer 1976).

If the lepton cross section scales (i.e., if $\mathrm{M}^{3} \mathrm{~d} \sigma / \mathrm{dM}$ depends only on $\mathrm{s} / \mathrm{M}^{2}$ ), then existing data at low $\mathrm{s}$ and low $\mathrm{M}^{2}$ can be used to predict $\sigma_{W}$ for large $\mathrm{m}_{\mathrm{W}}$. There is not enough data over a wide enough range of angles and energies to verify scaling directly. However, it is possible to find a model which scales and which when parametized in one region extrapolates correctly to another. This model is the quark-parton model, or Drel1-Yan model (Drel1 1970, Berman 1971), in which the lepton pair or $W$ meson is assumed to be created in a collision of elementary point-like constituents of the protons. The distributions of these quark 
constituents in the proton are required to be consistent with the observed deep inelastic electron scattering data.

However, these data are not sensitive to the so-called "sea" quark distributions for small values of $\mathrm{x}$ (the fraction of the proton momentum carried by the quark) and hence the dilepton data for large $s / M^{2}$ must be considered as determining the structure functions in this region, rather than checking the model.

Fig. II. 2 shows the experimental data compared with the preictions of the model for sea quark distributions assumed to behave like $(1-x)^{7}$ (Peierls 1977). It is evident that scaling is at least consistent; more recent data suggest that $(1-x)^{9}$ might give somewhat better agreement for large $s / M^{2}$. The extension of these measurements to ISABELLE energies is of profound importance in verifying this whole picture of proton structure and determination of the structure functions.

Given this model for the structure functions, the cross section for $W$ production can be explicitly calculated, the result being shown in Fig. II.3. The most important signature for a charged $W$ will be a lepton of high transverse momentum, up to one half the mass of the $W$. The branching ratio will be model dependent, and therefore also the width, since the coupling to leptons is completely fixed in the model. The principal backgrounds are likely to come from electromagnetically produced lepton pairs and from the decay of hadrons produced with large transverse momenta, the empirical ratio of leptons to hadrons in the latter case being $10^{-4}$. In Fi.g. II. $4 \mathrm{a}$ both backgrounds are shown. It is evident that the .ngle lepton spectrum can be expected to show a broad peak falling off sharply above $\mathrm{p}_{\perp}=\frac{1}{2} \mathrm{~m}_{\mathrm{W}}$ and slowly below, with the true backgrounds being relatively small.

The cross section for neutral $W^{\circ}$ production is also given in Fig. II.3. Although plotted as a function of $s / \mathrm{M}^{2}$, it does not strictly scale since in the Weinberg-Salam model the coupling strength and mass are related. However, the variation from 


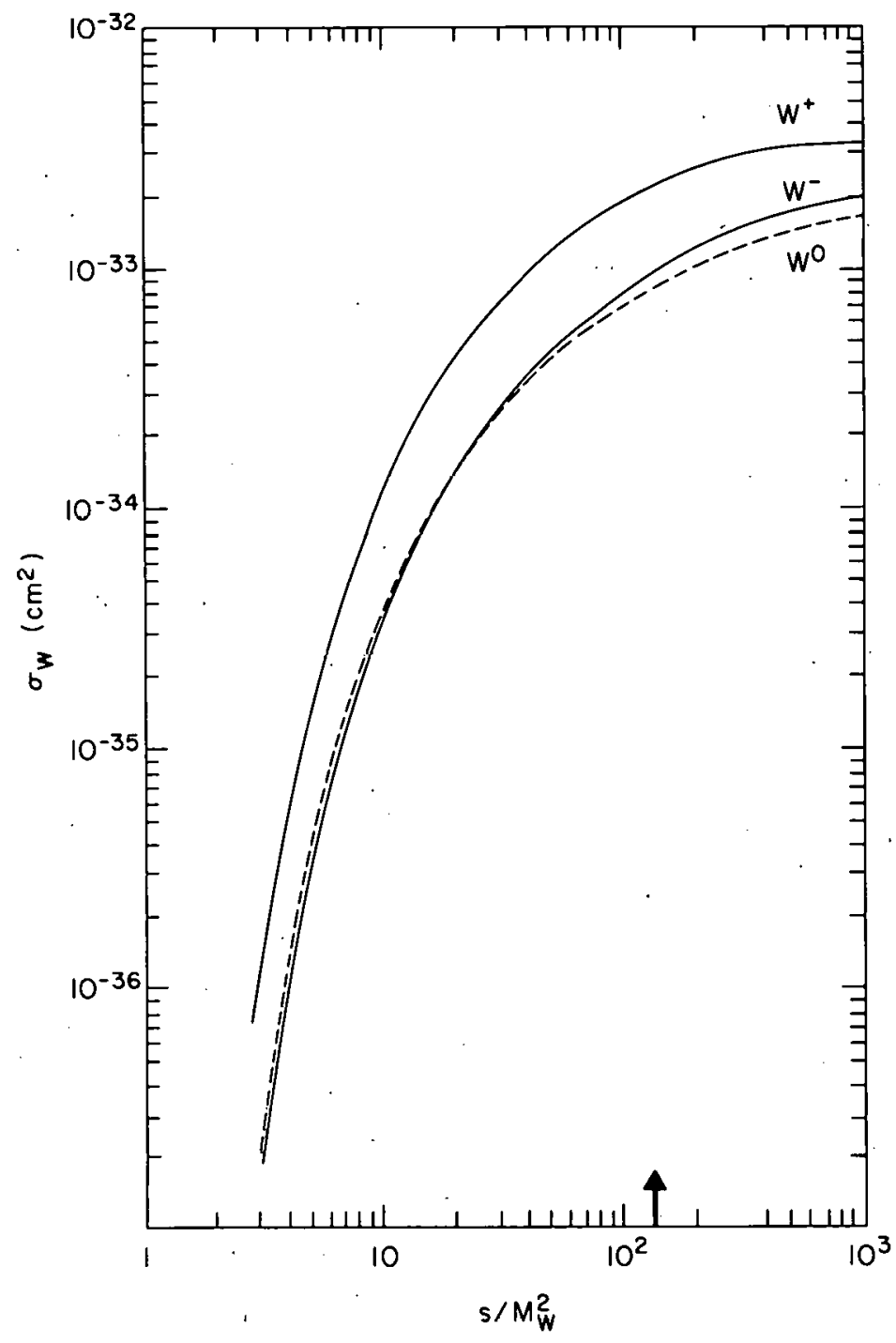

Fig. II.3. Quark-parton mode1 preciction for $W$ meson total production cross section. The model is that discussed in the text and responsible for the fit in Fig. II.2. Cross sections for $W^{ \pm}, W^{-}$and $W^{\circ}$ are shown. The $W^{\circ}$ curve does not strictly scale: a mass of 77 and $\theta \mathrm{W}$ of $39^{\circ}$ have been assumed. The arrow indicates the point corresponding to $\mathrm{M}_{W}=70$ and ISABELLE energy. 
scaling only involves a factor of order unity. Fig. II.4b shows the single lepton spectrum including the interference with the direct electromagnetic background for masses equal to and much larger than those given by the model. Fig. II.5 shows the predictions for the charge ratio at $90^{\circ}$ from this process. In the absence of any weak component the ratio would be 1 ; so this ratio, which is easier to measure than the absolute cross section, may well be a signature of the $W^{\circ}$ contribution even if the $W^{\circ}$ should be too massive to jserve directly.

issive Particle Production

The high energy available at ISABELLE is advantageous in searching for high-mass particles since generally in hadronic interactions the energy must be much larger than the threshold for the production to be significant. This has already been discussed in connection with the $W$ mesons. It can also be seen from the data on $\mathrm{J} / \psi$ production: the cross section rises very rapidly up to $\mathrm{s} / \mathrm{m}^{2} \approx 50$ and continues to increase up to the highest available energy (Cobb 1977). At ISABELLE large values of $\mathrm{s} / \mathrm{m}^{2}$ can be achieved for very large masses.

Massive, narrow vector mesons like the $\mathrm{J} / \psi$ are of particular interest because for each new quark $q$ there should be one or more such mesons made from a $q \vec{q}$ pair. Furthermore, they are relatively easy to detect because they decay into lepton pairs with substantial branching ratios. Thus the $J / \psi$ and the $\psi^{\prime}$ provided the first evidence for the existence of the charmed quark (Aubert 1974, Augustin 1974). Another pair of resonances, the $Y$ and $Y^{\prime}$, have recently men discovered (Herb 1977) and are generally believed to be bound lirs of a fifth type of quark having a mass of about $5 \mathrm{GeV}$.

Many models of weak interactions suggest that there should be at least one more quark to form a doublet with the $5 \mathrm{GeV}$ one, and more may exist. The number of quarks and their masses can be investigated at ISABELLE by looking for the vector mesons formed from them. It is believed that the proton contains very few heavy 


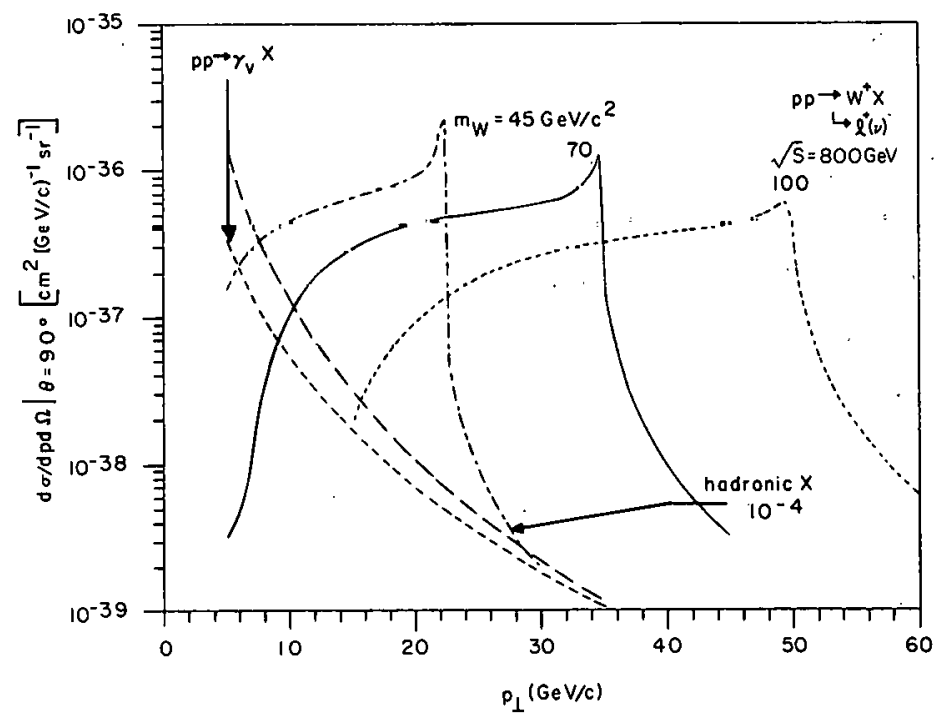

Fig. II.4a. Single lepton distribution at $90^{\circ}$ from $\mathrm{W}^{+}$production in the quark-parton model for various choices of $\mathrm{W}^{+}$ mass as a function of transverse momentum. The expected backgrounds are also shown from electromagnetic charged lepton pair production and from a vector gluon model of hadron production with lepton to hadron ratio $10^{-4}$ (See Fig. II.10). 


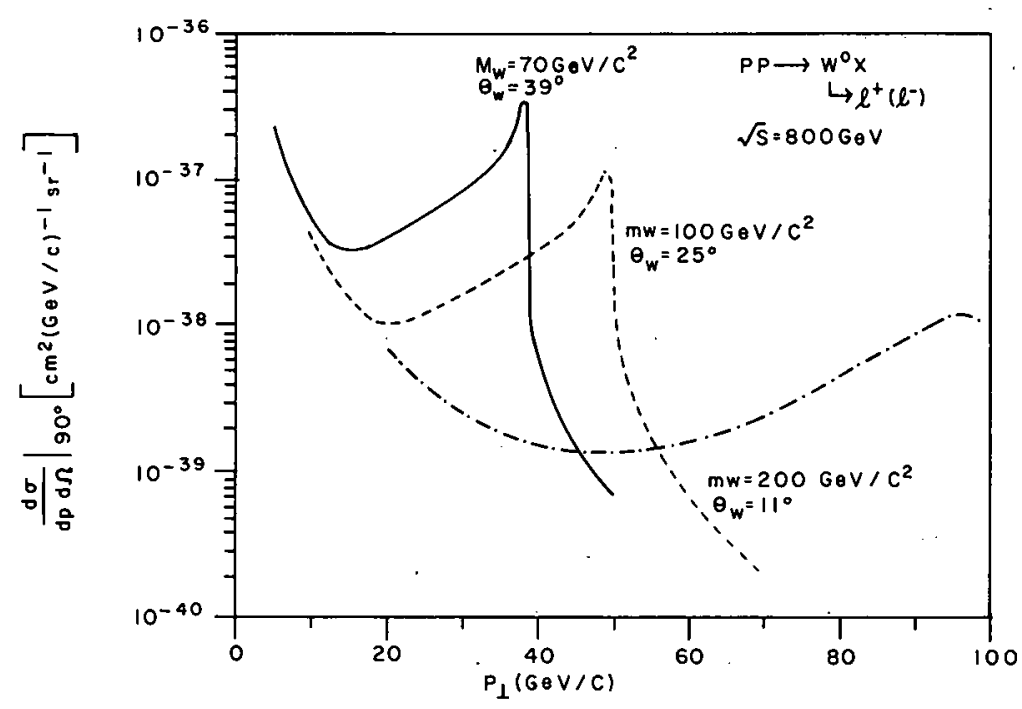

Fig. II.4b. Single charged lepton distribution at $90^{\circ}$ from $w^{\circ}$ decay according to the quark-parton model for various. combinations of $W^{\circ}$ mass and Weinberg angle $\theta_{\mathrm{W}}$. The curves all include background from and interference with direct electromagnetic lepton pair production. 


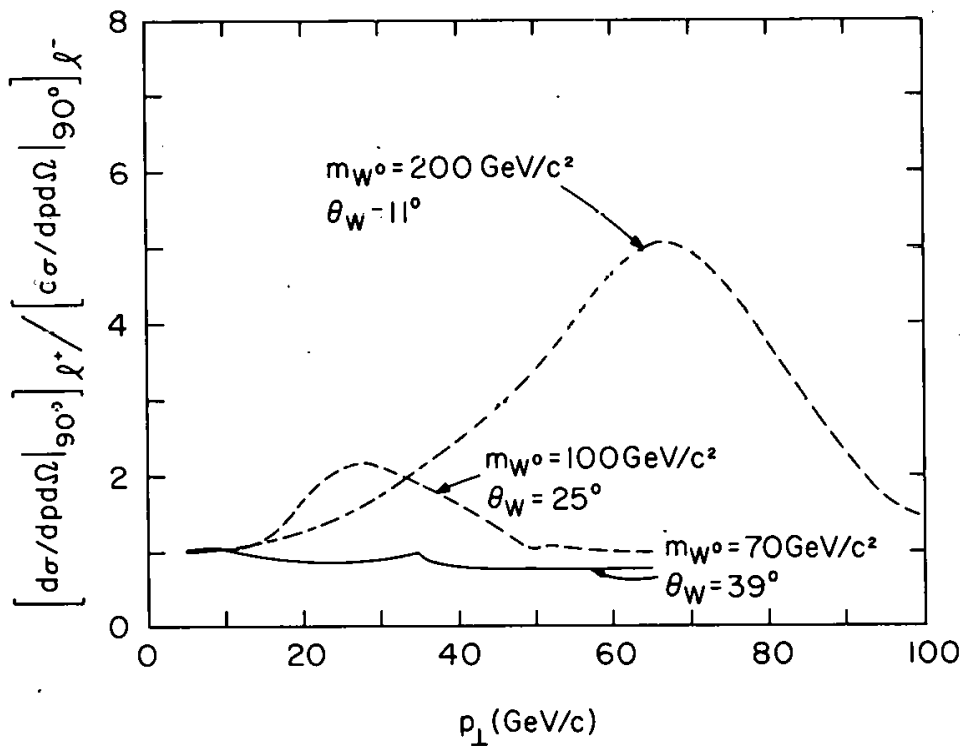

Fig. II.5. Charge ratio of positive to negative leptons from $w^{0}$ decay at $90^{\circ}$ as a function of transverse momentum, according to the quark-parton model. The parameters are the same as for the source curves in Fig. IT.4h and illustrate the charge ratio as an independent signal of $w^{O}$ production. 
quarks so that the cross sections for producing them in pairs can be related by scaling arguments. Then for a vector meson of mass $m$

$$
\left.\frac{\mathrm{d} \sigma}{\mathrm{dx} \mathrm{F}_{\mathrm{F}}}\right|_{\mathrm{x}_{\mathrm{F}}=0}=\frac{\Gamma_{\mathrm{n}}}{\mathrm{m}^{3}} \mathrm{~F}\left(\frac{\mathrm{s}}{\mathrm{m}^{2}}\right)
$$

where $\Gamma_{n}$, the width into normal hadrons not containing the new quark, provides a measure of the coupling to protons, and where $F$ is a universal function (Gaisser 1977). Hence the cross section or observing the meson in $\mathrm{pp} \rightarrow \ell^{+} \ell^{-} \mathrm{X}$ is

$$
\left.B_{U \ell} \frac{d \sigma}{d x_{F}}\right|_{x_{F}=0}=\frac{\Gamma_{\ell l}}{m^{3} B_{n}} F\left(\frac{s}{m^{2}}\right)
$$

where $\Gamma_{\mathcal{U}}$ is the leptonic width and $B_{n}=\Gamma_{n} / \Gamma_{\text {tot }}$.

The function $F$ can be determined from the energy dependence of the $\mathrm{J} / \Psi$ cross section and is shown in Fig. II.6. The observed cross section for the $Y$ then agrees with Eq. (2) if $\Gamma_{U \ell} \approx 0.4 \mathrm{KeV}$, in reasonable argument with the prediction for a charge $-1 / 3$ quark (Eichten 1977). The variation of $\Gamma_{\ell l}$ with the mass depends on details of the dynamics. Assuming that a hypothetical new meson has the same $\Gamma_{\mathcal{U}}$ as the $J / \Psi$, one can use Eq. (2) to estimate its production cross section at $\sqrt{s}=800 \mathrm{GeV}$ as a function of its mass. The envelope of the resonance peaks for $\Delta \mathrm{m} / \mathrm{m}=10 \%$ is shown in Fig. II.7. For a $50 \mathrm{GeV}$ meson

$$
\text { В }\left.\frac{\mathrm{d} \sigma}{\mathrm{dy}}\right|_{\mathrm{y}=0} \approx 4.7 \times 10^{-36} \mathrm{~cm}^{2} \text {, }
$$

rresponding to a rate of about $30 /$ hour for $|y| \leq 1$. Thus masses ? to at least $50 \mathrm{GeV}$ should be observable.

If additional quarks exist, then there should be multiplets of hadrons made from them and stable against all except weak decays. The cross sections for the production of such hadrons should increase rapidly with energy, and they may be quite large (Gaisser 1975). However, such hadrons would be expected to have short 


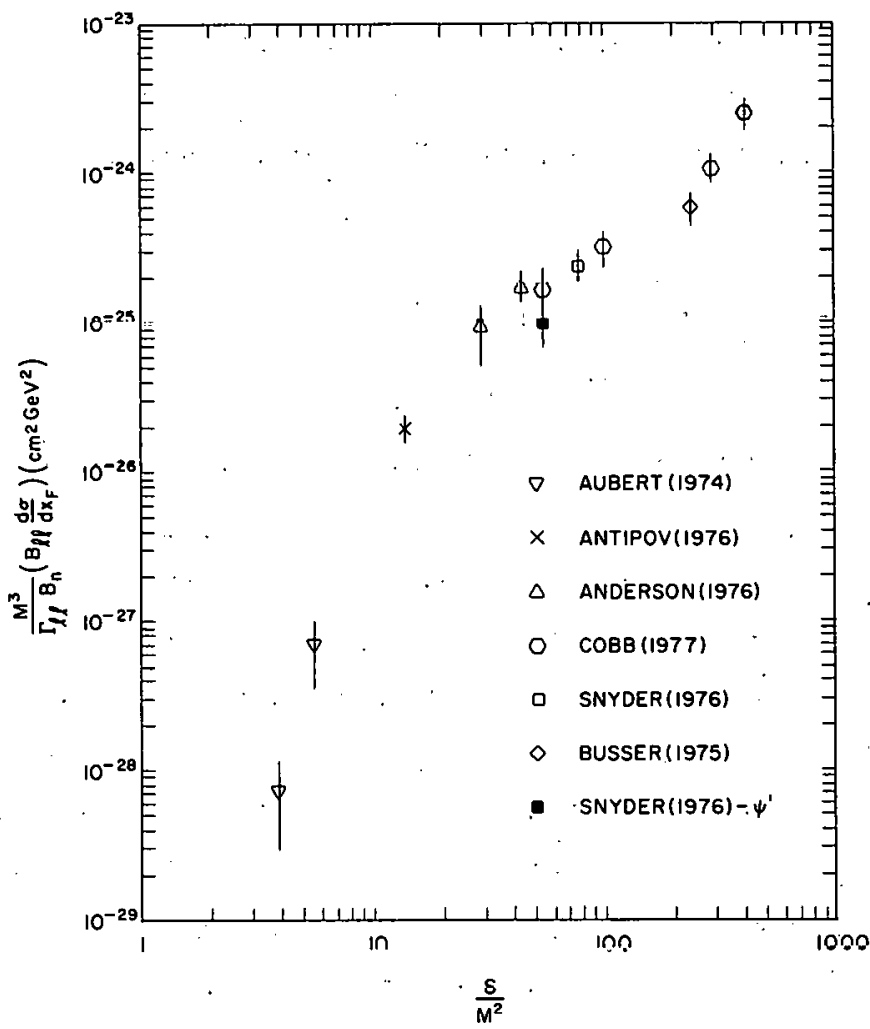

Fig. II.6. The scaling function $F\left(s / M^{2}\right)$ defined in Eq. (1). 


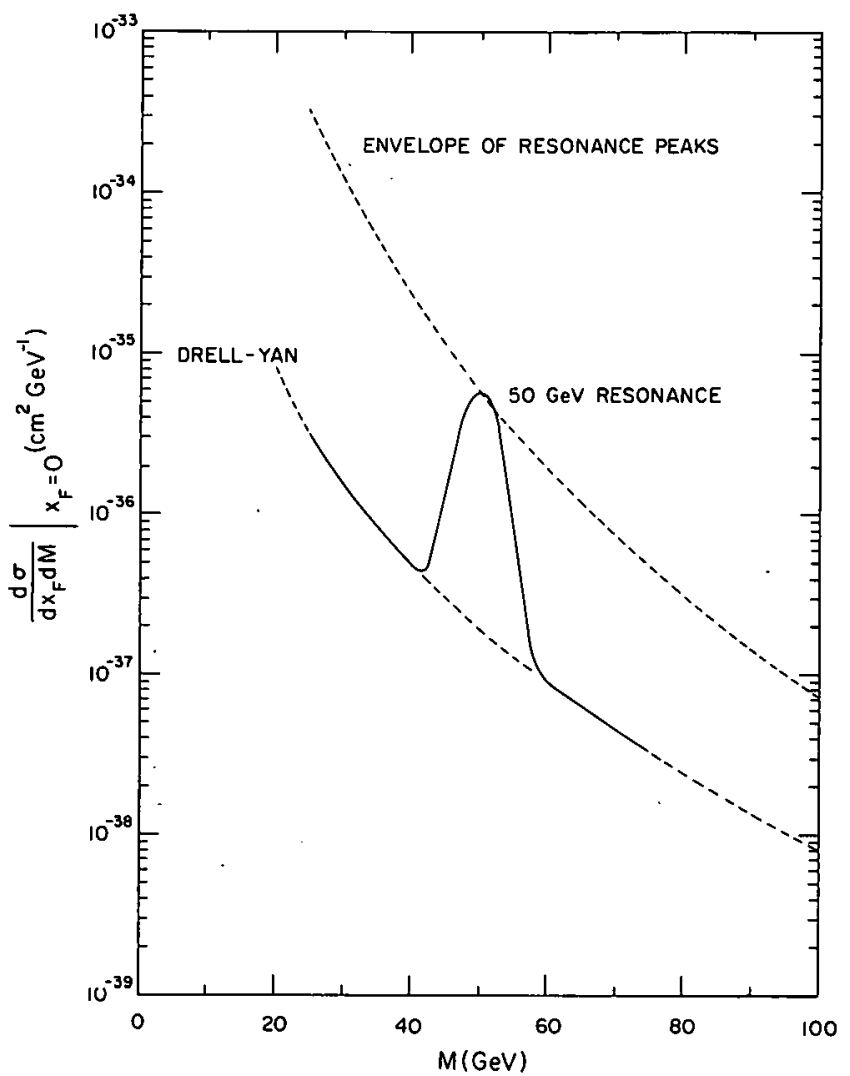

Fig. II.7. The predicted envelope of resonance peaks in $\mathrm{pp} \rightarrow \ell^{+} \ell^{-} \mathrm{X}$ for $\sqrt{\mathrm{s}}=800 \mathrm{GeV}$ and $\Delta \mathrm{M} / \mathrm{M}=10 \%$. The Drell-Yan background is calculated from the same model as Fig. II.2. The hypothetical $50 \mathrm{GeV}$ resonance shown would be observable at ISABELLE. 
lifetimes and to decay into many particles, so they will be hard to detect unless their decays provide some special signatures.

Another important massive particle is the Higgs boson (Weinberg 1976, Abers 1973) which is one of the ingredients in the unified gauge theories of weak and electromagnetic interactions. Its expected properties are not nearly as well defined as those of the W mesons: there may be several different ones or possibly none may exist at all. If they do exist, their masses must be greater than $4.9 \mathrm{GeV} / \mathrm{c}^{2}$ (Linde 1976, Weinberg 1976) and they are expected to cor more strongly to massive particles than light ones, making pp collisions a favorable place to look. Studies of their expected properties (E11is 1976) suggest that they do not have any striking signature. The mechanism for producing them is either via quarkquark annihilation, like the $W$ mesons, or by bremsstrahlung from $W$ production.

\section{Production of Hadrons at Large Transverse Momentum}

A characteristic signature of the presence of point-like constituents in the proton is the production of hadrons with large transverse momenta, reflecting the importance of small impact parameters in the elementary collisions of the constituents. More generally, one would think that large $\mathrm{p}_{\perp}$ processes are closely related to the short-distance structure of the proton. The first observation that hadron production cross sections decrease only like a power of $p_{\perp}$ rather than exponentialiy was made at the ISR several years ago (Büsser 1973). Since then large $p_{\perp}$ phenomena have been studied extensively (Frisch 1976, Fox 1976).

Since the cross section does decrease rapidly with $\mathrm{p}_{\perp}$, even $i$ much less rapidly than an extrapolation from small $\mathrm{p}_{\perp}$ would suggest, the existing data are limited to $\mathrm{p}_{\perp}<9 \mathrm{GeV} / \mathrm{c}$. However, the large $\mathrm{P}_{\perp}$ cross section is observed to rise rapidly with $\sqrt{ } \mathrm{s}$. Thus, ISABELLE with its high energy and good luminosity should substantially extend the accessible range of $\mathrm{P}_{\perp}$. 
This range can be estimated by considering the general class of hard scattering models. In these models the proton is assumed to contain constituents which initially have limited transverse momenta but which can scatter through large angles because they are pointlike. The sçattered constituents then fragment into hadrons. Such models typically lead to a one-particle inclusive cross section at large $\mathrm{p}_{\perp}$ of the scaling form

$$
E \frac{d \sigma}{d^{3} p}=\frac{1}{\left(p_{\perp}\right)^{n}} f\left(x_{\perp}, \theta\right)
$$

where $\theta$ is the center-of-mass angle and

$$
x_{\perp}=\frac{2 p_{\perp}}{\sqrt{s}}
$$

(Sivers 1976). The available data are generally consistent with this form with $n \approx 12$ for protons and $n \approx 8$ for other particles produced (Frisch 1976, Chicago 1977).

Various theoretical models within the hard scattering framework make different predictions for $n$ and for the form of $f\left(x_{\perp}, \theta\right)$ in Eq. (3). Perhaps the most natural model is based on the scattering of quarks by gluon exchange and gives $\mathrm{n}=4$ (Berman 1971). Other models involving different constituents or scattering mechanisms give values of $\mathrm{n}$ ranging from 8 to 12 (Gunion 1972, Field 1976).

An unprejudiced estimate of the values of $\mathrm{p}_{\perp}$ attainable at ISABELLE, can be obtained by using Eq. (3) and the pp $\rightarrow \pi^{\circ} x$ cross section from the ISR (Büsser 1973) to calculate cross sections as

function of $n$. For $\sqrt{s}=800$, the minimum $x_{\perp}$. observed at the ISR uld already correspond to $\mathrm{p}_{\perp}=32$ and a negligible cross section except for small $\mathrm{n}$. To be conservative Fig. II.8 gives the prediction for $\sqrt{s}=400$. It is certain that the ISABELLE cross sections would be higher, but the details will depend on as yet unobserved values of $x_{\perp}$. From the ISR experience and the high ISABELLE Iuminosity it is quite reasonable to expect to measure invariant cross 


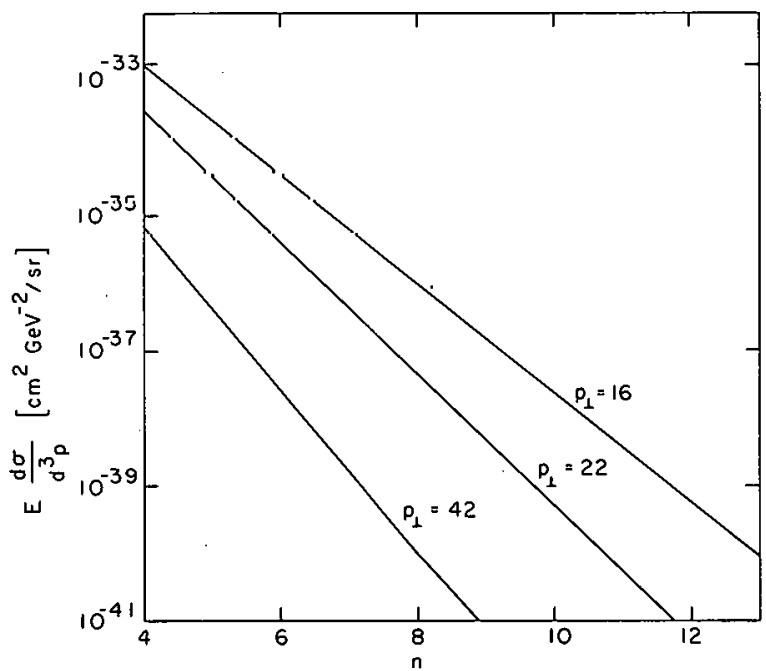

Fig. II.8. Effect of different scaling laws for hadron production at large $p_{\perp}$, for $\sqrt{s}=400 \mathrm{GeV}$ as a function of the scaling exponent $n$. The curves are adjusted to agree with the observation at ISR energies. 
sections of $10^{-38} \mathrm{~cm}^{2} \mathrm{GeV}^{-2} \mathrm{sr}^{-1}$. Thus one could reach $\mathrm{p}_{\perp}$ of $25 \mathrm{GeV} / \mathrm{c}$ if $\mathrm{n}=8$ continues to hold, and $\mathrm{p}_{\perp}$ of more than $40 \mathrm{GeV} / \mathrm{c}$ if $\mathrm{n}=4$ proves to be correct at larger $\mathrm{P}_{\perp}$.

Besides extending the range of $\mathrm{p}_{\perp}$, ISABELLE will be able to explore the region of small $x_{\perp}$ but large $p_{\perp}$. The CCR collaborations have fit their data with the form

$$
E \frac{\mathrm{d} \sigma}{\mathrm{d}^{3} \mathrm{p}}=\frac{1.54 \times 10^{-26}}{\left(\mathrm{p}_{\perp}\right)^{8.24}} \exp (-26.1 \mathrm{p} / \sqrt{\mathrm{s}}) \mathrm{cm}^{2} \mathrm{GeV}^{-2} \mathrm{sr}^{-1}
$$

This fit is shown in Fig. II. 9 for $\sqrt{s}=800 \mathrm{GeV}$. The small $x_{\perp}$ part of this curve is not based either on theory or on experiment. It will be interesting to explore the cross section in this region, which corresponds to the small x part of the quark distribution where the sea quarks are important. If the CCR fit continues to hold as $\mathrm{x}_{\perp} \rightarrow 0$, then at $\mathrm{p}_{\perp}=20 \mathrm{GeV} / \mathrm{c}$, for example, the cross section at infinite energy is only a factor of 3.7 greater than at ISABELLE.

At the same time as it extends the $x_{\perp}$ region to much smaller values than presently attainable for high $\mathrm{p}_{\perp}$ processes, ISABELLE can utilize its high luminosity over its entire energy range from $\sqrt{s}=60$ to $\sqrt{s}=800$ to overlap not only the ISR range in $x_{\perp}$, but a good portion of the FNAL range as well. This is a very important feature since it will permit a smooth continuation of the present data to the highest available energy. Not only is the overlap valuable for normalization purposes, but the continuous energy range at high luminosity is essential for seeing thresholds for lew processes which affect the $\mathrm{p}_{\perp}$ distribution, such as the proluction of new massive particles.

In addition to the single particle distribution at $90^{\circ}$, there are a great many other important high $\mathrm{P}_{\perp}$ studies that can be done at ISABELLE. For example, the type of particles produced can be identified for $\mathrm{p}_{\perp} \leqslant 20 \mathrm{GeV} / \mathrm{c}$, multiplicities of particles produced 


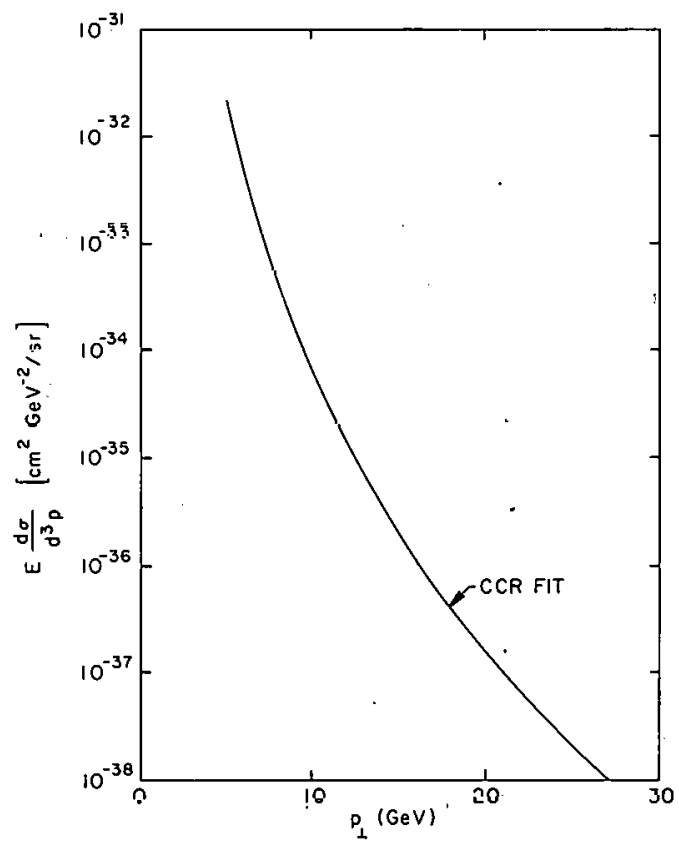

Fig. II.9. Extrapolation of observation of large $p_{\perp}$ production from ISR energies to ISABELLE energies using the CCR fit proportional to $\left(p_{\perp}\right)-8.24 \exp \left(-26.1 p_{\perp} / \sqrt{ } s\right)$. 
in conjunction with a high $\mathrm{p}_{\perp}$ particle can be measured, and correlations in momentum and angle between high $\mathrm{p}_{\perp}$ particles can be studied. These are all straightforward extensions of lower energy experiments already done at the ISR. An important question will be the study of the dependence on the production angle $\theta$ with which the high $\mathrm{P}_{\perp}$ particle emerges with respect to the beam direction. This should be a sensitive test for the production mechanism and structure functions of the proton.

\section{rets}

Confidence in the validity of constituent models has been greatly increased by the observation of jets in large $p_{\perp}$ events (British 1977, CERN 1977). It is believed that if the constituents are quarks, they will be prevented from escaping by a force which behaves roughly like an elastic string. When the quarks are sufficiently separated it becomes energetically favorable to break the string by producing hadrons, which should have small momenta transverse to the quark direction. Thus each quark fragments into a jet of hadrons with limited transverse momenta relative to the jet axis (Bjorken 1973). The unscattered constituents similarly produce hadrons with ordinary small transverse momenta.

The general features predicted by this picture have been confirmed by studies of correlations among particles with large transverse momenta (British 1977, CERN 1977). Given a high pl trigger, there is a positive correlation for finding another high $\mathrm{p}_{\perp}$ particle on the same side with a small momentum perpendicular to the trigger direction. This indicates a jet on the trigger side. On the oppoite side the high $\mathrm{p}_{\perp}$ particles are widely distributed in angle. swever, it is possible to define a jet axis for each event such that the high $\mathrm{p}_{\perp}$ particles have small momenta perpendicular to this axis: Furthermore, this axis is approximately coplanar with the trigger particle and the incoming protons. These results lead one to believe that high $\mathrm{p}_{\perp}$ phenomena are related to the hard scattering of elementary constituents of the proton. 
Most of the available data are at trigger momenta of $2-4.5 \mathrm{GeV} / \mathrm{c}$. ISABELLE should greatly extend the range of $\mathrm{P}_{\perp}$ over which such correlations can be studied. At higher $p_{\perp}$ the separation of the particles in the jets from those coming from the spectator constituents should be much cleaner. Furthermore, the validity of the scaling assumptions of the constituent models could be tested over a much wider kinematic range. This should help to clarify the nature of the constituents of the proton.

Since it is unlikely that a single particle carries all or almost all of the momentum of a jet, the cross section for the production of a jet with a given $p_{\perp}$ should be much larger than that for a single particle with the same $\mathrm{P}_{\perp}$. There is preliminary data from Fermilab Experiment E260 indicating that the jet cross section is larger by roughly the expected amount (Fox 1976). Of course, this means that one can reach larger values of $p_{\perp}$ by triggering on a jet rather than a single particle.

In constituent models the jet cross section is calculated by folding the constituent cross section with the constituent distribution functions for the incoming hadrons. Fig. II.10 shows the jet cross section at $90^{\circ}$ as a function of $\mathrm{P}_{\perp}$ for several models in which the basic constituent interaction is quark-quark scattering (Peierls 1976). All of these models have the same quark distribution as was used to estimate $W$ production above. In the first model the quarks interact by the exchange of massless vector mesons, or gluons. Since color is not essential here, it is assumed that there is only one gluon, whose coupling $\alpha_{\text {eff }}$ is independent of flavor. This model can also be used to calculate the one-pion invariant cross section, which behaves like $\mathrm{p}_{\perp}{ }^{-4} \mathrm{f}\left(\mathrm{x}_{\perp}\right)$. While this does not fit the existing data, one can normalize to the data at $\sqrt{s}=53 \mathrm{GeV}$ and high $\mathrm{p}_{\perp}$ to obtain

$$
\alpha_{\text {eff }}=0.06 \text {; }
$$

this value is used in Fig. II. 10 and subsequent figures. 


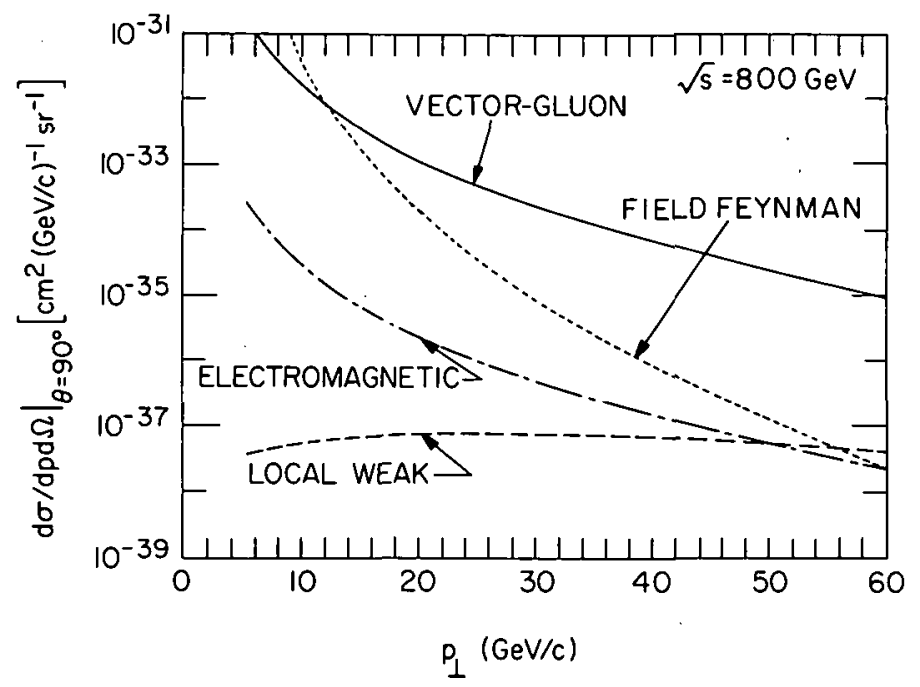

Fig. II.10. Quark-parton model prediction of single hadronic jet production at $90^{\circ}$ for various possible mechanisms. These include (a) vector gluon model normalized to the ISR data; (b) quark-quark scattering model of Field and Feyman; (c) electromagnetic production; (d) production by a local weak Fermi coupling, nonstrange charged currents only. 
The second model shown in Fig. II. 10 is a phenomenological one in which the quark-quark cross section is adjusted to fit the observed $p_{\perp}{ }^{-8} f\left(x_{\perp}\right)$ behavior of the one-pion cross section (Field 1976). The other two curves in Fig. II.10 are obtained from quark-quark scattering by electromagnetic and weak interactions.

Figure II.11 illustrates the energy dependence of jet production in the vector gluon model (Peier1s 1977). Evidently energies in the ISABELLE range are both necessary and sufficient to explore the interacting region of $\mathrm{p}_{\perp} \approx 50 \mathrm{GeV} / \mathrm{c}$ in which discrimination between different: models seems assiured.

It is also possible for a $W$ meson to decay into a pair of quarks and thus to produce two jets with large transverse momenta. The jets from the $W$ will probably be hard to isolate in the one-jet cross section, but they may be observable in the two-jet cross section because their angular distribution is different from that of jets coming from quark-quark scattering (Peierls, 1977). This is illustrated in Fig. II. 12, where the two-jet cross section is shown as a function of the angle of one jet for the angle of the other fixed at $90^{\circ}$. Evidently the possibility of observing the $W$ jets depends both on the size of the quark-quark cross section and on the accuracy with which the jet momentum can be determined. The detection of jets from $W$ decay seems clearly possible if the FieldFeynman model is correct, but rather difficult if the vector gluon model is right. While detection of the hadronic decay of the $W$ may be possible with careful measurements, it cannot be relied on for the discovery of the $W$.

\section{Energy Dependence of Strong Interactions}

The previous sections have emphasized the importance of ISABELLE for producing high-mass particles and for observing interactions at large $p_{\perp}$. Such experiments will seek to understand the nature of the constituents of matter. Of equal importance is the question of how the hadrons made from these constituents scatter 


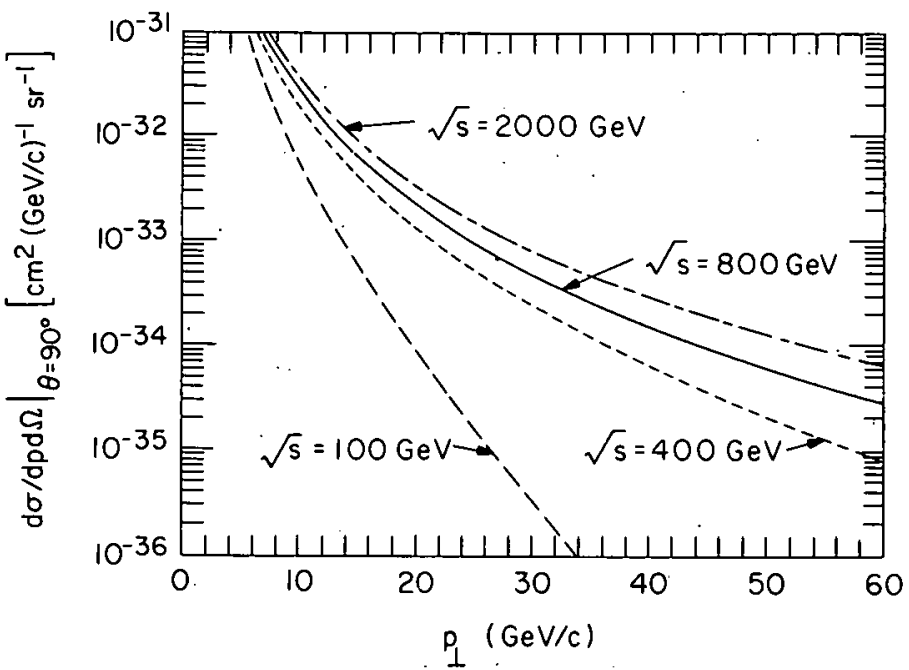

Fig. II.11. Single hadron jet production at $90^{\circ}$ as a function of transverse momentum for various values of total centerof-mass energy. The curves are derived from the quarkparton model together with, a vector gluon quark-quark interaction. 

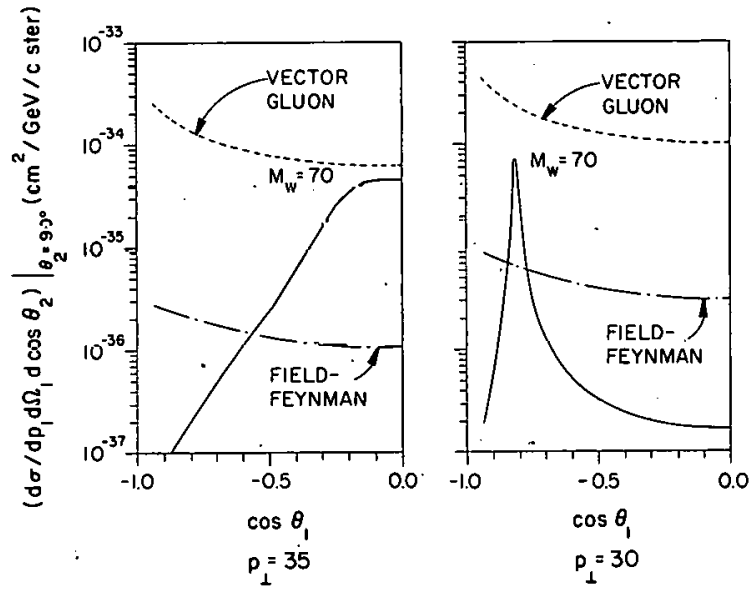

Fig. II.12. Angular dependence of double hadronic jet production in the quark-parton model as a function of $\cos \theta_{1}$, for $\theta_{2}=90^{\circ}$, where $\theta_{1}, \theta_{2}$ are the production angles of the two jets. The three mechanisms are: charged $W$ decay, $M_{w}=70 \mathrm{GeV}$, vector gluon interactions, and the Field-Feynman quark-quark scattering. (a) $\mathrm{p}_{\perp}=$ $35 \mathrm{GeV} / \mathrm{c}\left(\equiv \frac{1}{2} \mathrm{M}_{\mathrm{W}}\right)$ (b) $\mathrm{P}_{\perp}=30 \mathrm{GeV} / \mathrm{c}$. 
via the strong interaction. ISABELLE can help to answer this question by greatly extending the range over which the energy dependence of such processes can be measured.

The bulk of the total cross section consists of elastic scattering and multiparticle production at small momentum transfer $t$. [For the reaction $a+b \rightarrow c+\ldots, t$ is the invariant square of the four-momentum difference between $a$ and $c$. The term "tchannel" refers to the related reactions $(a+\bar{c} \rightarrow \bar{b}+\ldots)$.] The ata on such reactions are best described in the Regge.framework (Collins 1971). It is generally true that if $t$ is fixed and $s$ is large, the singularities in the complex angular momentum $\mathrm{J}$ for the t-channel will control the large s behavior. In particular, a pole at $\mathrm{J}=\alpha(\mathrm{t})$ gives an amplitude proportional to $\mathrm{s}^{\alpha(t)}$. The Regge or J-plane formalism is useful because the existing data can be described at least approximately by a few terms: the "Pomeron" and several secondary Regge poles. The latter all correspond to mesons which can be exchanged in the $t$-channel, and since their contributions to cross sections decrease roughly like $\mathrm{s}^{-\frac{1}{2}}$, they should be negligible at ISABELLE energies. The Pomeron is less well understood, but is viewed as the manifestation of the basic multiparticle production process of strong interactions. It corresponds to a singularity near $\mathrm{J}=1$ and hence to constant or. slowly varying cross sections.

The most basic question to be answered by ISABELLE in this area is whether the concept of the Pomeron and of slowly varying cross sections is correct, or whether, for example, the observed ise of the total cross section is a threshold effect (Gaisser 373). Of course, the question is more general than any model.

If the general idea of the Pomeron is confirmed, then it will be important to measure the $s$ dependence of various cross sections. The most naive model of the Pomeron, namely a simple Regge pole with $\alpha(0)=1$, accounts for many gross features of the existing data. For example, it gives constant total cross sections, scaling 
of inclusive cross sections (Mueller 1970), and short-range correlations (Mueller 1971). However, the naive model fails to describe many significant facts: its successes are only approximate. Furthermore, it is logically inconsistent (Brower 1972). A more sophisticated model is therefore required. Typically the s dependences predicted by such models differ only by powers of $\log s$ (Cheng 1970, Abarbane1 1974, Migdal 1974), so that accurate data over a wide energy range is needed to make any progress in understanding strong interactions. With its good performance from $\sqrt{\mathrm{s}}=60 \mathrm{GeV}$ to $\sqrt{\mathrm{s}}=$ $800 \mathrm{GeV}$ ISABELLE will provide such data.

Total Cross Section. Most models which fit the existing data predict that the total cross section will continue to rise throughout the ISABELLE energy range with an asymptotic behavior

$$
\sigma_{\mathrm{T}} \sim \sigma_{0}(\log \mathrm{s})^{\nu}, 0<\nu \leq 2
$$

Two typical phenomenological fits (Diddens 1974) having $\nu=1$ and $\nu=2$ are shown in Fig. II.13. The predicted rise is large in comparison with the variation in the existing data and reflects the large energy range which ISABELLE would make available.

If a smooth rise of such a magnitude is observed, it would be an important confirmation of our present general ideas about strong interactions. Furthermore; accurate measurements over the whole ISABELLE energy range should give a much clearer picture of the energy dependence of $\sigma_{T}$. This should lead to increased understanding, just as the FNAL and ISR data have shown that cross sections do not approach constants as predicted by the naive model of the Pomeron (Hendrick 1975).

Elastic Cross Section. After the total cross section, the elastic cross section is the simplest measure of strong interactions. The shape of the elastic cross section for small $t$ can be described by a slope parameter

$$
b=\frac{d}{d t}\left(\log \frac{d \sigma}{d t}\right)
$$




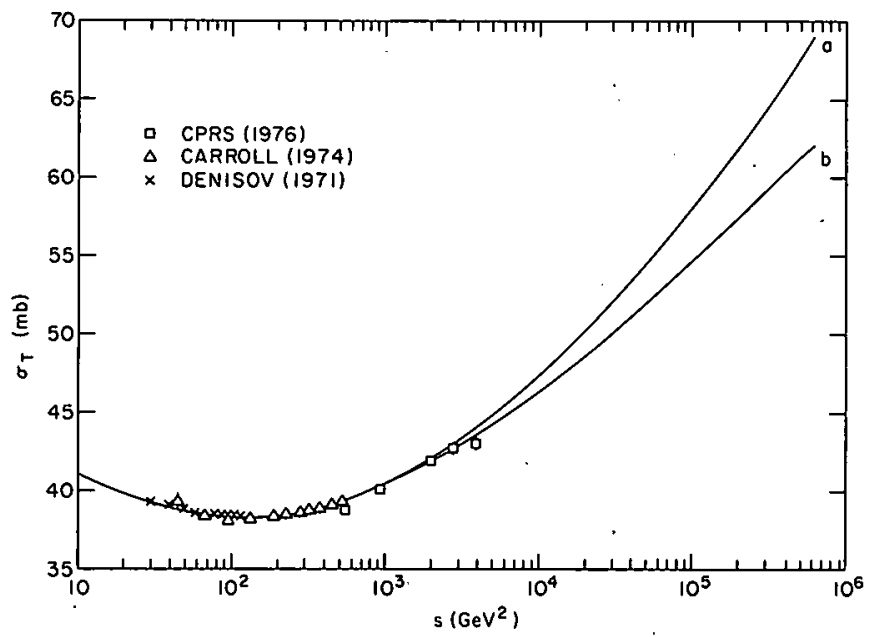

Fig. II.13. High energy data on pp total cross section and typical extrapolation to ISABELLE energies (Diddens 1974): (a) $\sigma_{\mathrm{T}} \sim$ const $\times \log ^{2} \mathrm{~s}$;

(b) $\sigma_{T} \sim$ const $\times \log s$. 
This is found to be different for $|\mathrm{t}| \& 0.15(\mathrm{GeV} / \mathrm{c})^{2}$ and $|\mathrm{t}|>$ $0.5(\mathrm{GeV} / \mathrm{c})^{2}$ (ACHGT 1972); data in both regions are shown in Fig. II.14. From the obvious inequality $\sigma_{\mathrm{el}}<\sigma_{\mathrm{T}}$ one finds that if $\sigma_{\mathrm{T}}$ behaves as in Eq. (5), then the average slope satisfies

$$
b>\frac{\sigma_{0}}{16 \pi}(\log s)^{\nu}
$$

Asymptotically an increasing $\sigma_{T}$ implies an increasing $b$. In practice the slope is much larger than this bound and so represents quite independent information. The form of the fit shown in Fig. II.14 is motivated by the simple Regge pole model of the Pomeron and interesting deviations from it might reasonably be expected over the ISABELLE energy range.

At $t \approx-1.4(\mathrm{GeV} / \mathrm{c})^{2}$ the elastic cross section has a striking dip which appears between $s=200 \mathrm{GeV}^{2}$ and $s=400 \mathrm{GeV}^{2}$ (see Fig. II.15). This dip is suggestive of a diffraction minimum from a proton of wel1-defined geometrical shape. Three models, all incorporating this general view but predicting different positions for the dip and heights for the secondary maximum, are shown in Fig. II.15. ISABELLE should determine whether a geometrical interpretation is correct or whether the dip is caused by an "accidental" zero which disappears at higher energy.

At still larger $|t|$ the data shown in Fig. II.16 suggest the possibility of a second dip.

Other exclusive reactions $c$ an also be studied at ISABELLE. The cross sections for $\mathrm{pp} \rightarrow \mathrm{pN}^{*}$ and $\mathrm{pp} \rightarrow \mathrm{N}^{*} \mathrm{~N}^{*}$ are approximately energy independent (Pavia 1975, Webb 1975, CHOV 1976b), so these should be observable. A detailed study of their energy dependence would complement that of elastic scattering. Reactions involving quantum number exchange such as $p p \rightarrow \Delta^{++} \Delta^{0}$ fall rapidly with energy, but if they can be observed they would extend the study of secondary Regge terms to a new energy range. 


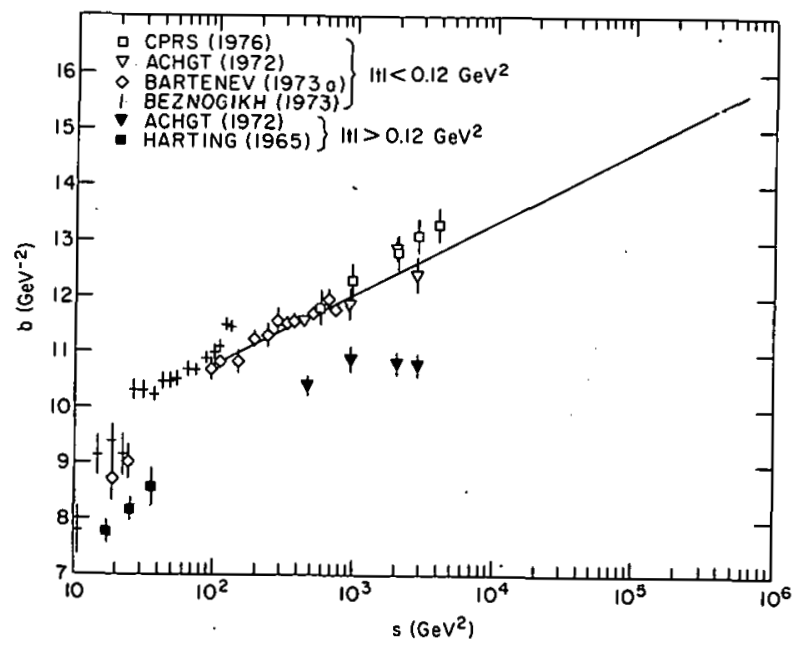

Fig. II.14 Data on the elastic slope parameter b. The equation for the curve drawn is: $b=8.23+0.556 \log s$; this form is appropriate to a Pomeron which is a simple Regge pole (Bartenev 1973a). 


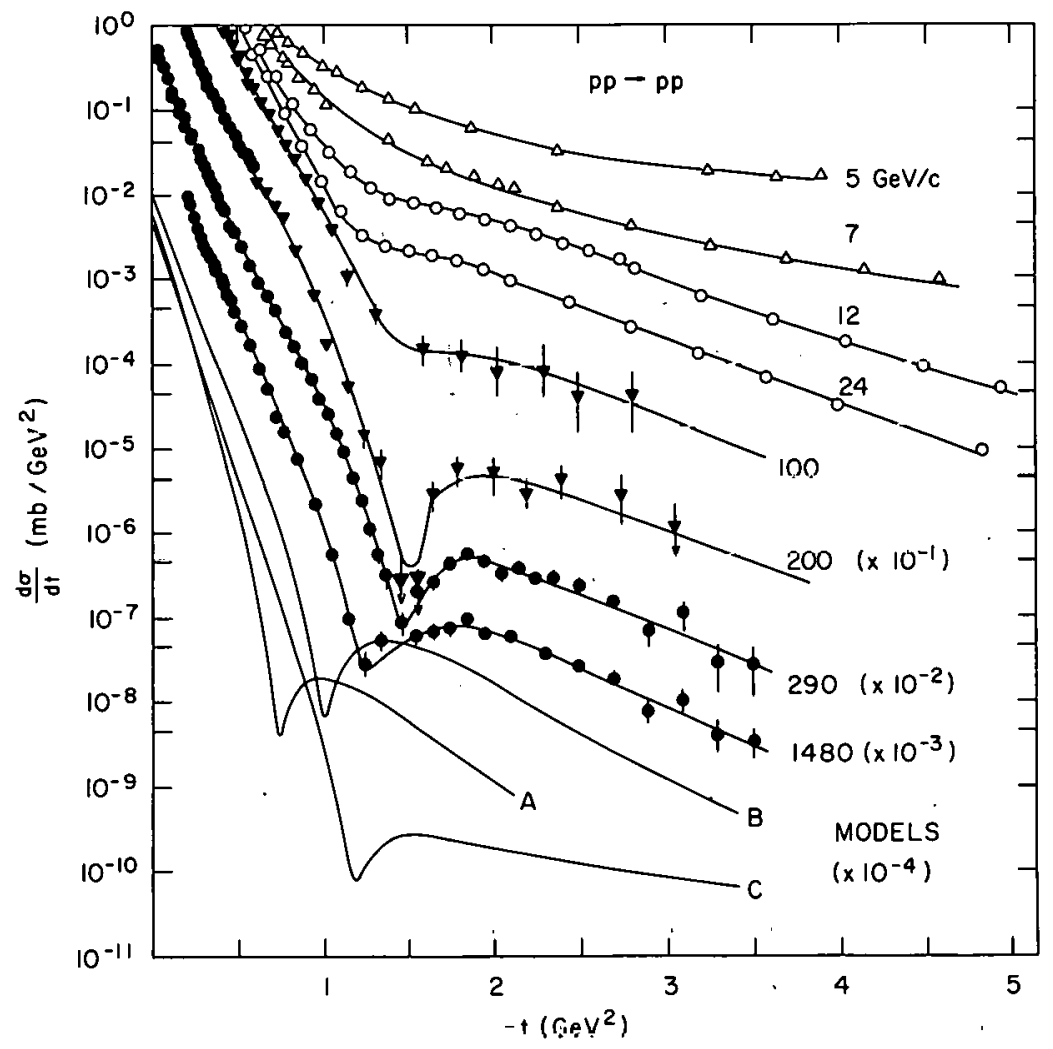

Fig. 11.15. Elastic pp cross section for moderate $t$ showing the dip structure. The data compilation is from Amaldi 1976. The three tuodel predictions for $\sqrt{s}=800 \mathrm{GeV}$ are (A) Geometrical scaling using curve (a) in Fig. II.13 for $\sigma_{\mathrm{T}}$ (Dias de Deus 1973); (B) Coupled-channel Regge-eikonel móde1 with $\alpha(0)>1$ (Crozier 1976); (C) Regge-eikonel model with $\alpha(0)>1$ (Chu 1976). 


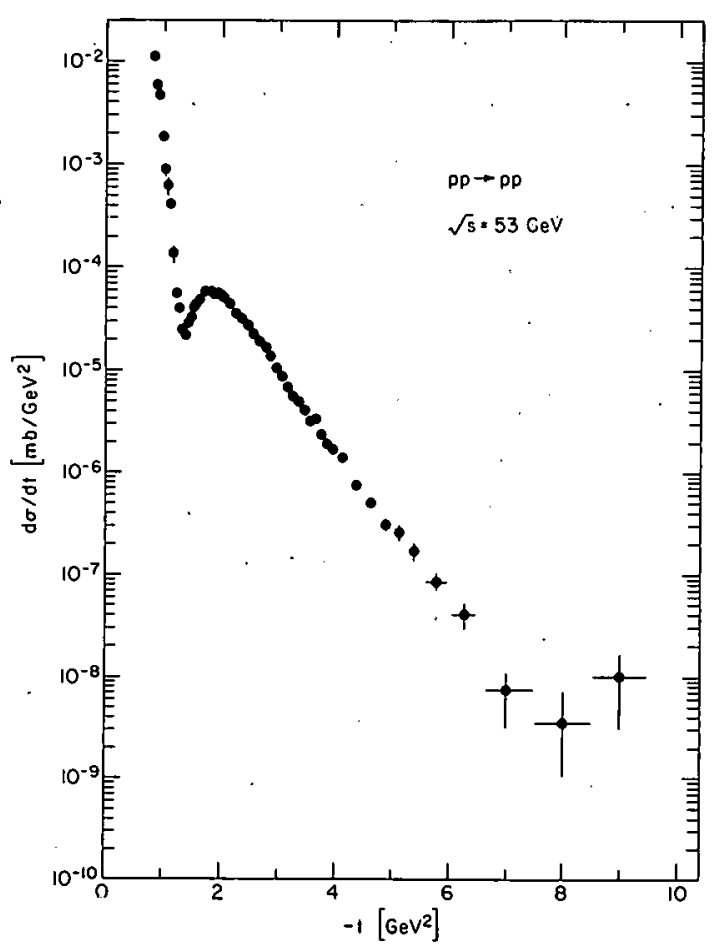

Fig. II.16. Elastic pp crose section at large $\mid$ to for $\sqrt{s}=53 \mathrm{GeV}$ (CHOV 1976a). The cross section at ISABELLE energies should be similar. 
Real Part of Forward Amplitude. It is generally believed that the elastic scattering amplitude $f(s, t)$ is an analytic function in the cut $s$ plane and satisfies a dispersion relation. It follows from this and the optical theorem that

$$
\rho(s)=\operatorname{Ref}(s, 0) / \operatorname{Imf}(s, 0)
$$

can be calculated given a knowledge of $\sigma_{T}$. It can also be measured by Coulomb interference. As can be seen from Fig. II.17 the available data are consistent with the calculated values. The high- $\beta$ intersection of ISABELLE will permit such measurements to be exten to $\mathrm{s}=6.4 \times 10^{5} \mathrm{GeV}^{2}$. This will provide a sensitive test of dispersion relations in an entirely new energy range.

\section{One-Particle Inclusive Reactions}

One-particle inclusive reactions provide a measure of the average behavior of multiparticle production, which accounts for about $80 \%$ of the total cross section. At ISABELLE, it would be interesting to measure the cross sections for

$$
\mathrm{p}+\mathrm{p} \rightarrow \mathrm{c}+\mathrm{X},
$$

for $c=\pi^{ \pm}, \mathrm{K}^{ \pm}, \mathrm{p}^{ \pm}$and as many other particles as possible. The Lorentz invariant cross section for such reactions is

$$
E \frac{d \sigma}{d^{3} p}=\frac{1}{\pi} \frac{d v}{d y d p_{\perp}^{2}}
$$

and is best described as a function of three variables: $s$, the rapidity $\mathrm{y}=\log \left(\mathrm{E}+\mathrm{p}_{\|}\right) /\left(\mathrm{E}-\mathrm{p}_{\|}\right)$, and $\mathrm{p}_{\perp}{ }^{2}$. In some regions $x=2 p_{\|} / \sqrt{s}$ is a more useful variable than $y$.

It is useful to divide the available range of $y$,

$$
-\frac{1}{2} \log \frac{s}{m^{2}}<y<\frac{l}{2} \log \frac{s}{m^{2}}
$$

into a central region and two fragmentation regions. The fragmentation regions occupy about two units at each end of the range of $y$, 


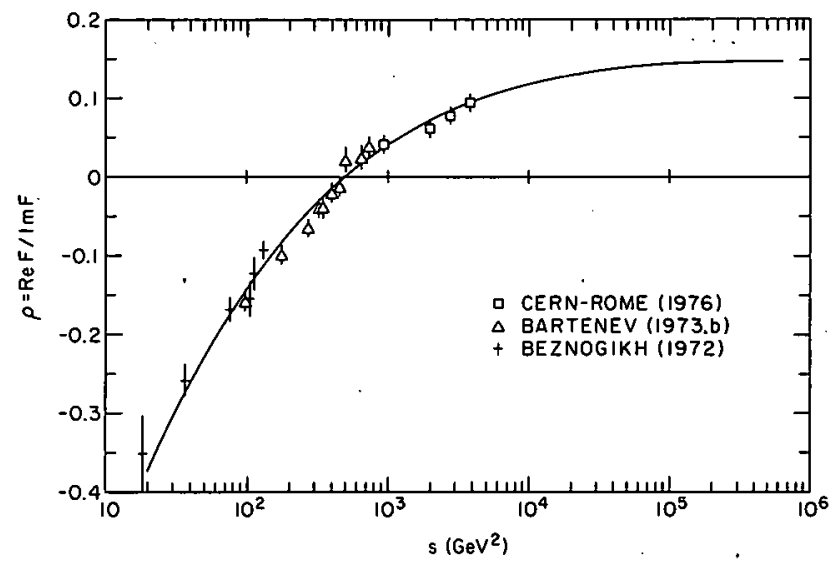

Fig. II.17. Data on $\rho(s)=\operatorname{Ref}(s, 0) / \operatorname{Imf}(s, 0)$ as measured by Coulomb interference. The curve is a dispersion-relation calculation using curve (a) of Fig. II.13 for $\sigma_{T}$. 
with the central region lying between them (Lillethun 1973). Thus, the length of the central region is

$$
\begin{aligned}
\Delta y & =4 \text { at ISR } \\
\Delta y & =9.5 \text { at ISABELLE }
\end{aligned}
$$

This expansion of the central region is of substantial benefit in studying inclusive reactions.

The naive Pomeron model predicts that $E\left(d \sigma / d^{3} p\right)$ is independent of $\mathrm{s}$ and is also independent of $\mathrm{y}$ in the central region (Mueller 1970). These predictions are only approximately correct. In particular, the cross section at $y=0$ increases substantially over the ISR energy range, aṣ is shown in Fig. II.18 (British 1976).

In a wide class of models the inclusive cross section in the central region is of particular interest because it isolates the exchange of a single Pomeron (Abramovskii 1972, Cheng 1973). The $\mathrm{s}^{\alpha-1}$ fit in Fig. II.18 is motivated by such models, but the power $\alpha=1.16$ is larger than expected. Data at higher energy should help to clarify this energy dependence.

It is also interesting to measure inclusive cross sections near the edge of phase space, $x \approx 1$. In this region the tripleRegge model (Abarbanel 1971) provides at least an approximate description of the existing data (Field 1974). This model relates the inclusive cross section to a total cross section for Reggeonparticle scattering at a center-of-mass energy equal to the missing mass $M$. Since $M^{2}=(1-x) s$, at ISABELLE for $x=0.98$ one could reach $M^{2}=12800\left(\mathrm{GeV} / \mathrm{c}^{2}\right)^{2}$ which is much larger than $s$ at the ISK. By studying the inclusive cross section for $x \approx 1$, one could thus learn to what extent the rise observed in the pp cross section also occurs in the Reggeon-proton cross section.

\section{Correlations}

The very high energies available at ISABELLE would be particularly useful in studying both correlations between particles 


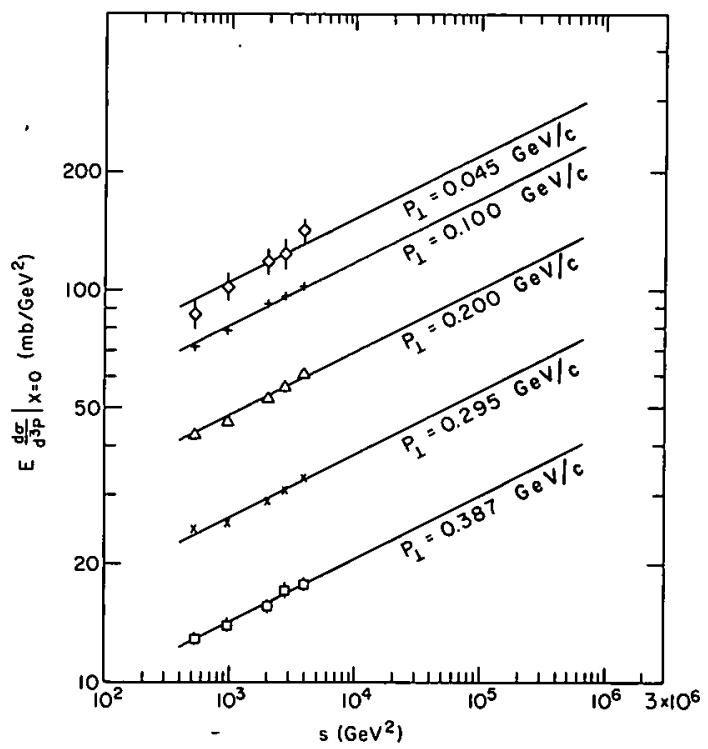

Fig. II.18. Energy dependence of $\mathrm{pP} \rightarrow \pi^{-} \mathrm{X}$ cross section at $x=0$ (British 1976). The naive Pomeron model predicts that this cross section is constant. 
in the central region and correlations between a proton near $\mathrm{x}=1$ and particles in the missing mass.

In the central region it is useful to distinguish long-range and short-range correlations. In the Mueller-Regge mode1, shortrange correlations are associated with secondary Regge poles, while long-range ones are associated with Pomeron cuts, or, more generally, with any nonfactorizable part of the Pomeron (Quigg 1973). In other models, short-range correlations come from clusters or similar effects, while long-range correlations can arise from the production of particles off multiple chains or from absorption (Botke 1973). Additional long-range correlations are introduced by energymomentum conservation, especially near the edge of phase space.

Contour plots of the correlation function

$$
R\left(\eta_{1}, \eta_{2}\right)=\left(\sigma_{\text {inel }} \frac{\mathrm{d} \sigma}{\mathrm{d} \eta_{1} \mathrm{~d} \eta_{2}} / \frac{\mathrm{d} \sigma}{\mathrm{d} \eta_{1}} \frac{\mathrm{d} \sigma}{\mathrm{d} \eta_{2}}\right)-1
$$

as a function of the pseudorapidity $\eta=-\log \tan (\theta / 2)$ are shown in Fig. II.19. The short-range correlation depending mainly on $\left|\eta_{1}-\eta_{2}\right|$ is clearly evident in the central region. For $\eta_{1} \approx \eta_{2} \approx$ $\log s$ the correlation function becomes negative as required by energy-momentum conservation.

At ISABELLE, the size of the central region will be more than twice that at the ISR. Particles can be separated by more than one correlation length (about two units of rapidity) while remaining in the central region. This should make it possible to separate dynamical long-range correlations from both the short-range correlations and the trivial ones due to energy-momentum conservation. The high energy available at ISABELLE would also facilitate the study of correlations between a particle near $x=1$ and particles in the missing mass. In the triple-Regge model such correlations are related to inclusive reactions for Reggeon- 

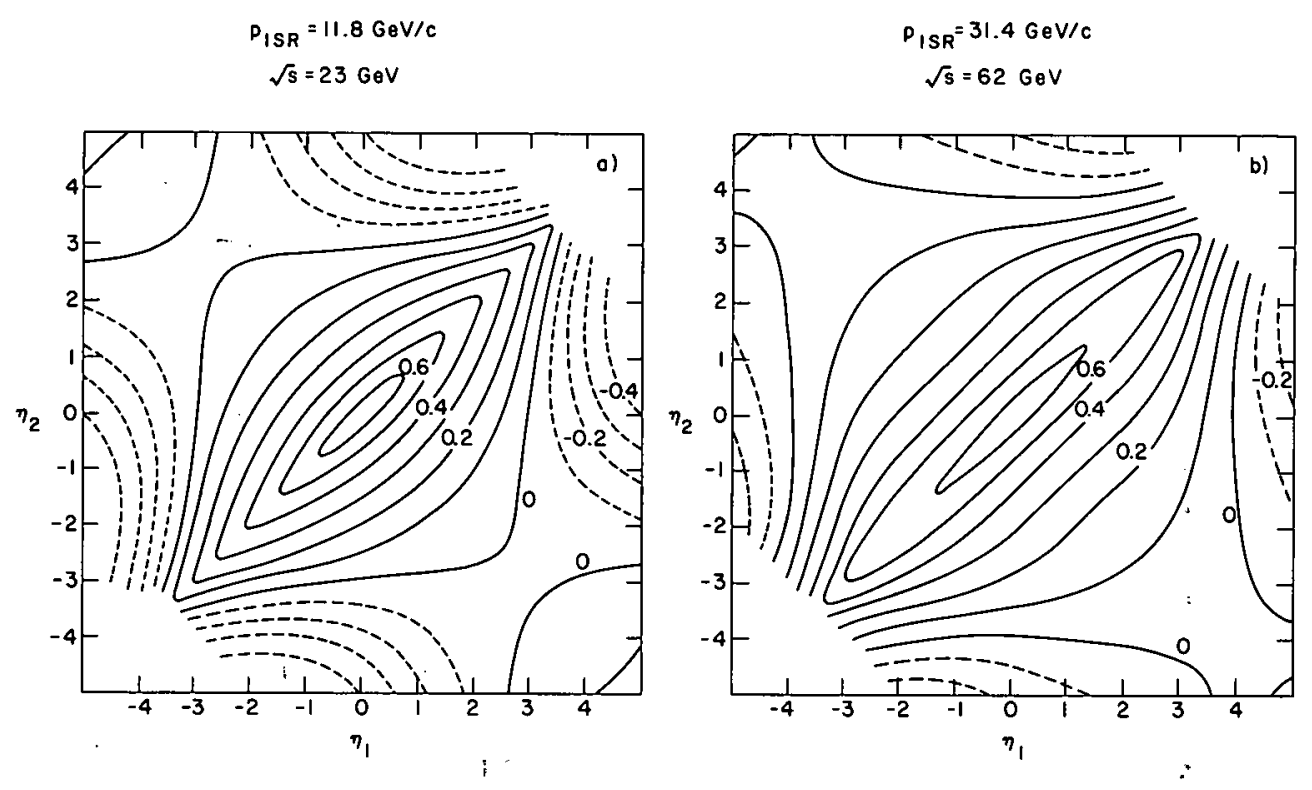

Fig. II.19. Contour plots of charged-particle correlation function $R\left(\eta_{1}, \eta_{2}\right)$ vs pseudorapidity $\eta=-\log (\tan \theta / 2)$. Note that the correlation is mainly short-range for small. $\eta_{1}$ and $\eta_{2}$, depending on $\left|\eta_{1}-\eta_{2}\right|$. 
particle scattering (Frazer 1973). Thus one would be able to study Reggeon-proton inclusive reactions at values of $\mathrm{M}^{2}$ comparable to the values at the ISR. 
Experiments will be carried out in the insertions in the ring lattice that are described in Section III.2. In designing the insertions particular emphasis was placed on providing the flexibility needed for accommodating the varied requirements of the many experiments that the physics objectives of ISABELLE imply. The 1975 Summer Study and 1976 and 1977 Workshops have confirmed that the present design of the insertions can indeed satisfy the experimental :equirements.

Al1 the experiments considered could be set up with the standard 60-meter free space available at the crossing point. Most experiments can utilize the standard beam focus at the insertion. For higher luminosity, the strength of the quadrupole doublets can be changed to reduce $\beta_{v}^{*}$ at the crossing point. This can yield luminosity of $4.5 \times 10^{32} \mathrm{~cm}^{-2} \mathrm{sec}^{-1}$. For proton beams of smallest angular divergence the opposite change is needed to increase $\beta_{v}^{*}$ at the crossing point. This is an essential requirement for the experiment to measure the real part of the forward scattering amplitude, for which $\beta_{v}^{*} \approx 75$ is required.

For some experiments the proton beams must pass through analyzing magnets. This must be done in such a way that they introduce no net dispersion into the circulating proton beams. In other words, for each dipole magnet in the iusertion there must be another compensating magnet that bends in the opposite direction. This is demonstrated in the Large Aperture Spectrometer described in this section. A similar dipole magnet sequence can $\geq$ used to change the crossing angle of the intersecting beams. rour common dipoles are required to provide luminosity of $10^{33}$ $\mathrm{cm}^{-2} \mathrm{sec}^{-1}$ by reducing the crossing angle by a factor of two. The free space for that configuration would be $30 \mathrm{~m}$. With a similar arrangement but with the polarities in the four dipoles reversed, the crossing angle can he increased. This feature can provide 
a short interaction diamond ( $\approx 40 \mathrm{~mm}$ long) which is desirable-for some experiments (Willis 1976).

Furthermore, the storage rings can be operated at unequal beam energies. It would be possible to maintain a fixed $p_{\perp}$ acceptance downstream from the crossing point and map out the $s$ dependence by changing the energy of the other beam. Unequal beam energies can also be used to check on possible systematic errors by maintaining a fixed $s$ with different combinations of beam energies.

A few'simple experiments, such as a monopole search (Giacomell 1975) using Makrofol-E and Nitrocellulose sheets, and a search for fractionally charged quarks (Foley 1975) have been discussed, but in general the experimental arrangements to fully utilize the potential of ISABELLE appear to be quite substantial. Some typical proposed experiments are described below.

\section{Lepton Detector}

A crucial test of the theory of weak interactions is the existence or nonexistence of the intermediate vector boson. As discussed previously, $W$ mesons should give a clear experimental signature by their leptonic decays and they may also be observable through their decays into hadronic jets. Experimental designs for both of these decay modes have been studied.

A design of a large Lepton Detector was carried out originally at the 1975 Summer Study (Burnstein 1975), and a reduced scale des1gn was considered at the 1976 Summer Workshop (Imlay 1976). This detector was designed to look for the most characteristic signature of $\mathrm{W}^{ \pm}$and $\mathrm{z}^{\circ}$ production, namely either single leptons or dileptons of high transverse momentum. In addition, since this detector will explore a new region of physics, it is designed with the capability of identifying both electrons and muons. The general configuration of the reduced scale lepton detector is shown in Figs. II.20a and b. An alternative arrangement was discussed in the 1977 Workshop (McDonald 1977). 


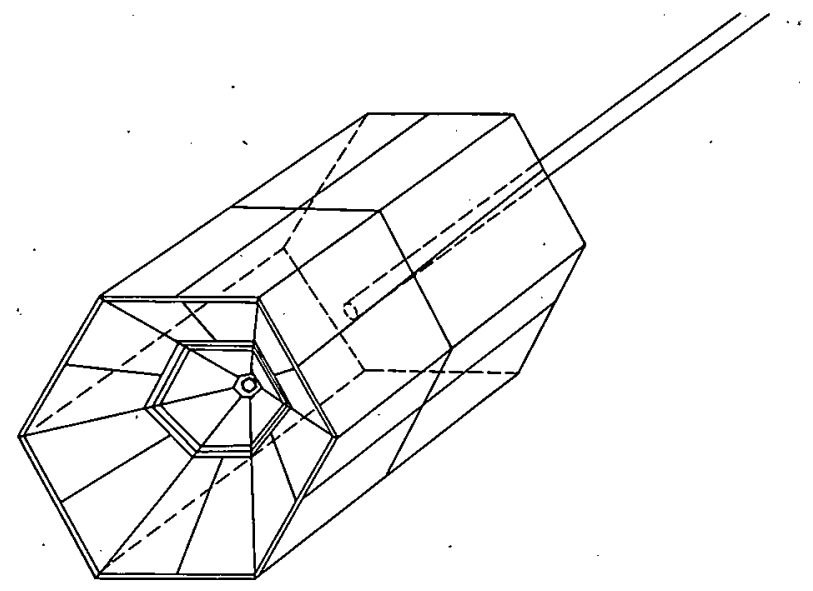

Fig. II.20a. Isometric view of large lepton detector (Burnstein 1975).

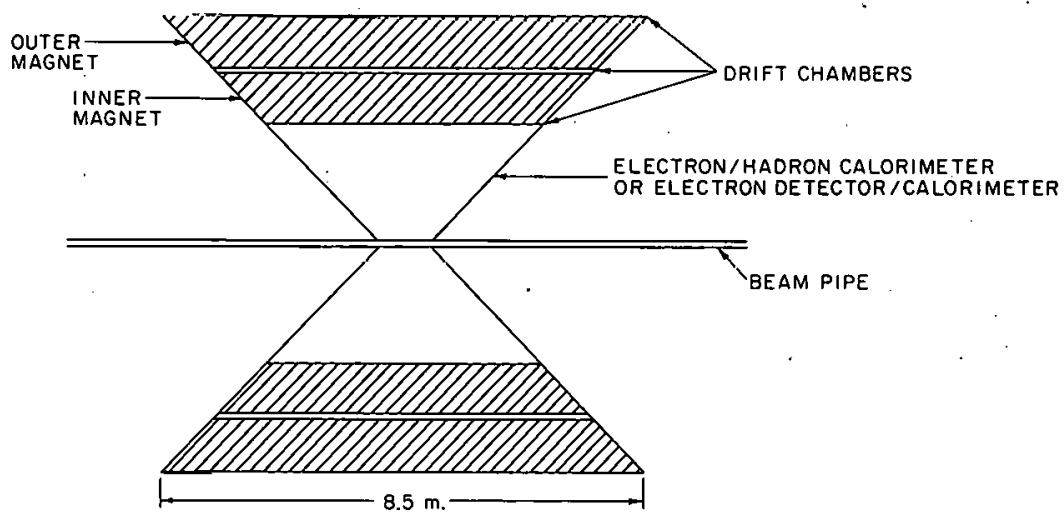

Fig. II.20b. Sectional view of lepton detector (Burnstein 1975). 
Electron identification would be carried out with a combination of transition radiation detectors and a liquid argon ionization chamber calorimeter. An extremely efficient transition radiator has been constructed that consists of stacks of thin lithium foils followed by a xenon-gas filled MWPC to detect the $x-r$ ays from the radiator (Fischer 1975). Such a radiator-detector system has been extensively tested and is being used for electron identification in an experiment at the ISR with a geometry very similar to the one described here.

The optimum performance of the electron identifier is determined by extrapolating the performance of the ISR detector to T.SABELLE energies. The detector unit is optimized for the energy regime between 10 and $50 \mathrm{GeV}$, by using a lithium foil thickness of $0.0032 \mathrm{~cm}$ and a foil separation of $0.051 \mathrm{~cm}$. With this geometry 500 foils yield a detected $\mathrm{x}-\mathrm{ray}$ intensity $40 \%$ larger than the ISR detector. This is sufficient to offset the $30 \%$ increase in the hadron energy deposition so that the $\pi$-e separation remains the same as in the ISR detector, namely about 200 to 1 .

The calorimeter is composed of two cylindrical units, the inner une upllmlzed for electrons and the outer one for hadrons. The calorimeter is a liquid argon ionization chamber with a large number of plates to minimize the sampling fluctuations while keeping the average density high. For the electron section, 20 radiation lengths of converter are-needed, which amounts to $36 \mathrm{~cm}$ of steel or $11 \mathrm{~cm}$ of lead. For the hadronic section an additional 8 interaction lengths would be added to what exists in the first. sectiun, glving a cocal of $1 U . b$ interaction lengths. This will be composed of 100 plates of steel, $1 \mathrm{~cm}$ thick, separated by $0.5 \mathrm{cl}$ of liquid argon. The expected electron energy resolution is then $5 \% / \sqrt{\mathrm{E}}$, while for the hadron it will be $20 \% / \sqrt{\mathrm{E}}$, with $\mathrm{E}$ in units of GeV.

The outer section of the detector uses magnetized iron, both as a shield against hadrons and as a momentum measuring apparatus 
for muons up to $50 \mathrm{GeV} / \mathrm{c}$. The magnetized iron consists of two concentric hexagonal sections interspersed with drift chambers. A measurement of the muon momentum can be made to an accuracy of roughly $12 \%$. The inner detector elements do not have the capability, in each sector, to identify electrons by transition radiation and have the full electron and hadron calorimeters. There are two different types of modules. One contains the transition radiators and the electron calorimeter and the other contains only the elecron and hadron calorimeters. We can consider an initial experi-lent comprised of alternating types of modules. In detecting the muonic decay of the $W$, the complete solid angle is effective, while for the electronic decay there is a reduction, but by less than a factor of 2. Clearly any we correlations are also reduced by the same factor in this initial configuration. Depending upon the early performance of the detector and the early physics results, one has the option of changing the modular configuration.

The transition radiation detector and electron calorimeter section would detect the electron emerging from the $W$ decay while at the same time it rejects hadrons with good sensitivity. The transition radiation detector in conjunction with the calorimeter should, at $\mathrm{p}_{\perp}>10 \mathrm{GeV} / \mathrm{c}$, discriminate against pions at the level of $10^{-4}$ while still detecting most of the leptons. Also, one should note that most of the rates are from very low momentum particles and can be excluded if one is interested only in high $\mathrm{PL}$ particles by raising the electron calorimeter threshold and introducing a $p_{\perp}$ cut-off on the electrons accepted in the trigger. For ^xample, one expects only $\sim 2000$ pions per second with momentum

$5 \mathrm{GeV}$ in the entire detector at a luminosity of $10^{33} \mathrm{~cm}^{-2} \mathrm{sec}^{-1}$, compared with an estimated total counting rate of $10^{8}$ per second. The e-T separation of approximately $10^{4}$ to 1 can be achieved by having an electron identifier which can detect leptons with less than $1 \%$ pion background provided that the calorimeter, occupying thc middle section of the detector, can achieve another factor of 
100 in the hadron-electron separation.

Counting rates for $W$ detection can be estimated from the model calculation described in Section 2. For example, from Fig. II.4a the cross section for the peak in the transverse momentum spectrum for a $W$ mass of $70 \mathrm{GeV} / \mathrm{c}^{2}$ is $\mathrm{d} \sigma / \mathrm{d} \mathrm{p}_{\perp} \mathrm{d} \Omega=9 \times 10^{-37} \mathrm{~cm}^{2}$ $(\mathrm{GeV} / \mathrm{c})^{-2} \mathrm{sr}^{-1}$ for each lepton channel. The cross section is relatively flat around $90^{\circ}$. For a $2 \pi$ solid angle and a momentum spread of $15 \mathrm{GeV} / \mathrm{c}$ the resulting rate is about two counts per second for a luminosity of $10^{33} \mathrm{~cm}^{-2} \mathrm{sec}^{-1}$. The rate decreases with the mass of the $W$, dropping by about one order of magnitude for a $W$ mass of $200 \mathrm{GeV} / \mathrm{s}^{2}$.

A serious constraint on the design of the entire detector is the desirability of keeping transverse dimensions as small as possible to reduce the muon background in the outer muon detector resulting from the $\pi-\mu$ decays. In addition, the volume of the muon identifier grows rapidly with the transverse dimension and the amount of iron in it is a dominant factor in the total cost. The transition radiation detector is considered a "plug-in-unit" that could be removed and replaced by shielding, making the overall apparatus a muon detector with an even better $\pi \rightarrow \mu$ decay rejection.

The detection of small-angle particles by means of end caps should be left to a later phase of the experimental program. Initially, the lepton detector would concentrate on particles of high $\perp \perp$ and relatively large angle, without attempting to observe all secondary particles or achieve overall energy or momentum balance. End caps to detect small-angle particles could be added after a first round of experiments in which the lepton detector would cover angles within $\pm 45^{\circ}$ of the $90^{\circ}$ direction. Initial information on small-angle particles would come from experiments at other insertions with equipment designed for that purpose.

The observation of intermediate boson production for boson masses up to the order of $200 \mathrm{GeV} / \mathrm{c}^{2}$ should be possible. If, 
contrary to expectation, the masses were even higher than this, or if no $W$ bosons exist, then the apparatus described would still be able to observe weak interactions of the type pp $\rightarrow e v+e t c$. despite their smal1 cross section [of the order of $10^{-36} \mathrm{~cm}^{2}$ integrated for (ev) masses above $\left.60 \mathrm{GeV} / \mathrm{c}^{2}\right]$.

\section{Large Aperture Spectrometer}

This large detector system would also be capable of seeing weak interaction effects, and in particular, observing the $W$ boson $\mathrm{n}$ both its charged and neutral states. The Large Aperture Spectrometer (White 1976) emphasizes the detection of charged particles, with identification, and the measurement of photons from hadron decay. Given a high efficiency of hadron detection, the transverse momentum of the missing neutrino can be inferred and serves as a good signature of the weak decay. If the $W$ is produced moving slowly in the laboratory system, then both the charged lepton and the neutrino will generally have high transverse momentum and may be separable from the hadrons in the production process. Knowledge of these two momenta allows the calculations of the charged $W$ mass directly.

A more difficult but also attractive possibility is to see the $W$ decay into hadronic jets, and therefore, measure the branching ratio. It is not clear whether these jets can be distinguished from "normal" hadronic jets by their angular distribution and by their invariant mass distribution, but this possibility was borne in mind in designing the Large Aperture Spectrometer.

Apart from the $W$, there is the field of charmed particle spectroscopy which would be studied at ISABELLE. At high production lergies, in nucleon-nucleon collisions the $\mathrm{J} / \Psi$ has been identified at BNL, the ISR and at FNAL through the two lepton decay mode, but not directly by decay into hadrons. The higher mass charmed bosons couple less strongly to lepton pairs, and if the spectroscopy is to be pursued, the decays into hadrons must be seen. The large acceptance and good momentum resolution of the Large Aperture 
Spectrometer would provide a good handle on the multiparticle decays of charmed particles.

Furthermore a program of "conventional" hadron physics can be carried out with an apparatus like this one. In addition to the experiments discussed above there are a number of other processes which require high energies either to overcome phase space limitations or else to provide information about energy dependence. Examples include double diffractive dissociation, multiperipheral processes, and quasi two-body reactions such as $p p \rightarrow \Delta^{++} \Delta^{0}$. All of these are known to occur at lower energies.

Fig. II. 21 shows a schematic of the spectrometer system. In the forward direction the high momentum hadrons would be measured in the forward angle tagging spectrometers FATS 1 and FATS 2 (on the other side, not shown). The disintegration products of the incident protons and the particles that are produced in the central region can be differentiated. These two spectrometers can measure momenta of charged particles and the energy of the neutrals that are emitted within the cone of acceptance, without necessarily using any equipment inboard of their main deflection magnets. The measurement of inclusive particle production within a fairly extensive forward cone can be accomplished by these two spectrometers in a stand-alone running mode.

Each FATS spectrometer has a single bending magnet with a peak field integral of $90 \mathrm{kG}-\mathrm{m}$. The spatial resolution $\Delta x$ to be specified for the drift chambers ( $D$ in the figure) is $100 \mathrm{H}$. This gives us a resolution of $\Delta p / p^{2} \sim 0.5 \Delta x(\Delta x$ in meters, $p$ in $\mathrm{GeV} / \mathrm{c}$ ) which at $100 \mathrm{GeV} / \mathrm{c}$ and $\Delta \mathrm{x}=10^{-4}$ gives $\Delta \mathrm{p} \sim 0.5 \mathrm{GeV} / \mathrm{c}$. This figur is typical of the magnitude of the charged particle momentum uncertainty that we can expect.

The operating principle of the two wide angle spectrometers (WASP) is identical to FATS in that the detection is all downstream of the bending magnet. The $\int \mathrm{Bdl}$ of each magnet is $10 \mathrm{kG}-\mathrm{m}$ giving 

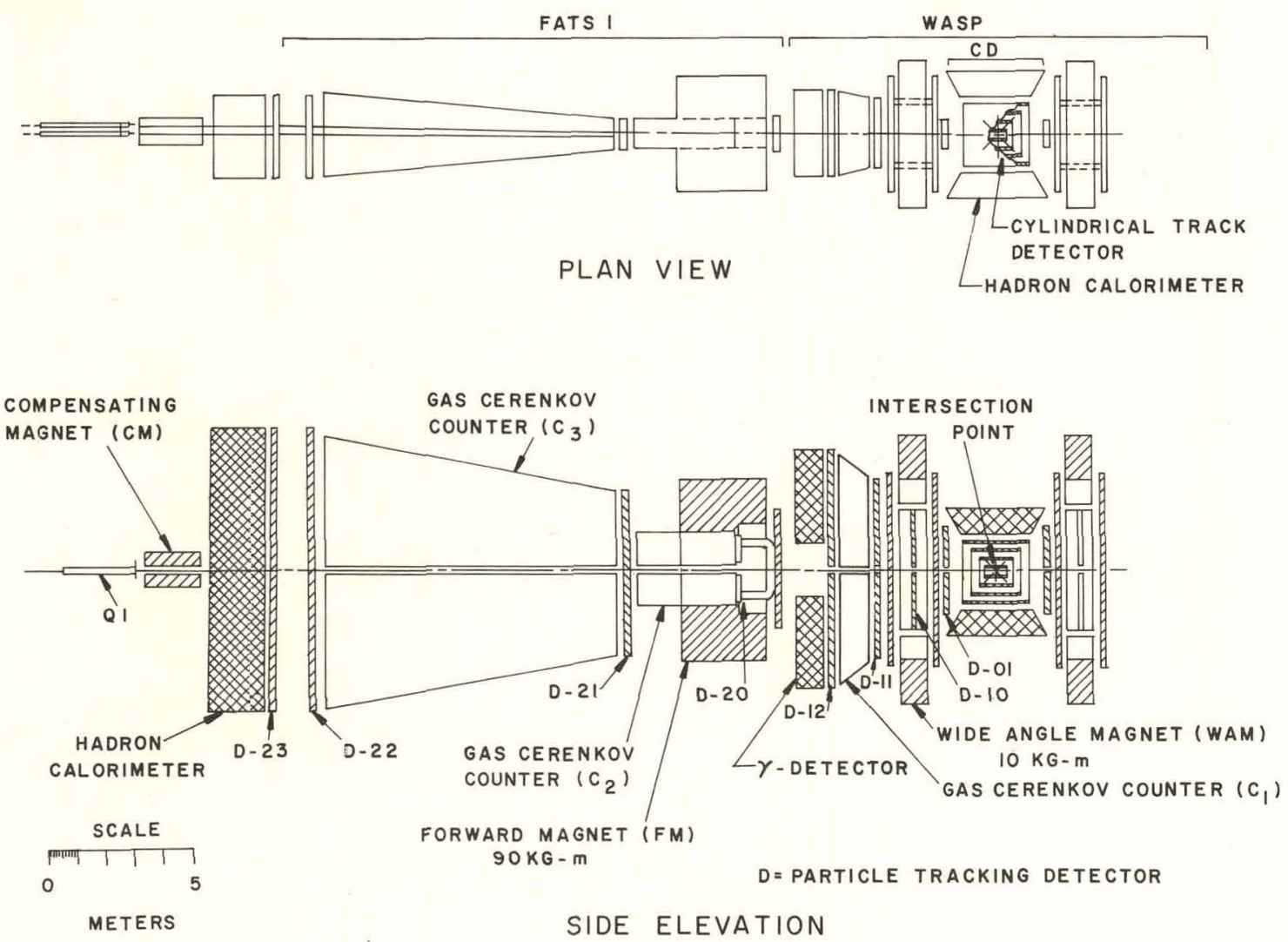

Fig. II.21. Large Aperture Spectrometer system consisting of forward angle tagging spectrometer, FATS, wide angle spectrometer pair, WASP, and central detector (White 1976). 
$\Delta \mathrm{p} / \mathrm{p}^{2} \sim 20 \Delta \mathrm{x}$ without the interaction point and $\Delta \mathrm{p} / \mathrm{p}^{2} \sim 4.8 \Delta \mathrm{x}$ with a measurement of the interaction point to the same spatial precision as elsewhere. Using this more precise number, below $3.8 \mathrm{GeV} / \mathrm{c}$ the material ahead of the spectrometer will dominate the momentum resolution, keeping it to $0.5 \%$. The large aperture magnet is of conventional type, although large in size. The magnet CM compensates the deflection of the ISABELLE beam before reentering the ISABELLE lattice, satisfying the present ISABELLE lattice design requirement. Neutral detectors will measure energy, and the charged particles have their momentum measured with the particle type identified. There are many technically advanced detectors which have been considered for the area around the interaction region itself. One utilizes a cylindrical geometry with its axis approximately parallel to the ISABELLE beam. A set of cylindrical proportional chambers which incorporate a longitudina1 readout, a calorimeter and/or shower detector and possibly a Cerenkov counter could be installed to detect large angle particles with momentum $\leqslant 20 \mathrm{GeV} / \mathrm{c}$.

The design attempts to establish a balance between charged particle measurement and identification and the difficulties of neutral particle reconstruction. For the luminosity of $10^{32} \mathrm{~cm}^{-2}$ $\mathrm{sec}^{-1}$, the rate of production of charged $\mathrm{W}^{\prime} \mathrm{s}$ has been estimated at $10^{4}$ /day. With the large aperture system this represents a more than adequate yield.

\section{Study of Hadrons at Large Transverse Momentum}

The recent discovery of charmed particles and the growth of the evidence in favor of the existence of jets in hadron-hadron collisions make a study of high $p_{\perp}$ hadrons of great current inter $\epsilon$ Such an experiment can be carried out in the setup shown in Fig. II.22.

It consists of two spectrometers of large solid angle (Peoples 1976), the axes of which are perpendicular to the colliding beams. The multiparticle acceptance for a jet of particles emitted near $90^{\circ}$ with respect to the colliding beams is good. The experiment 


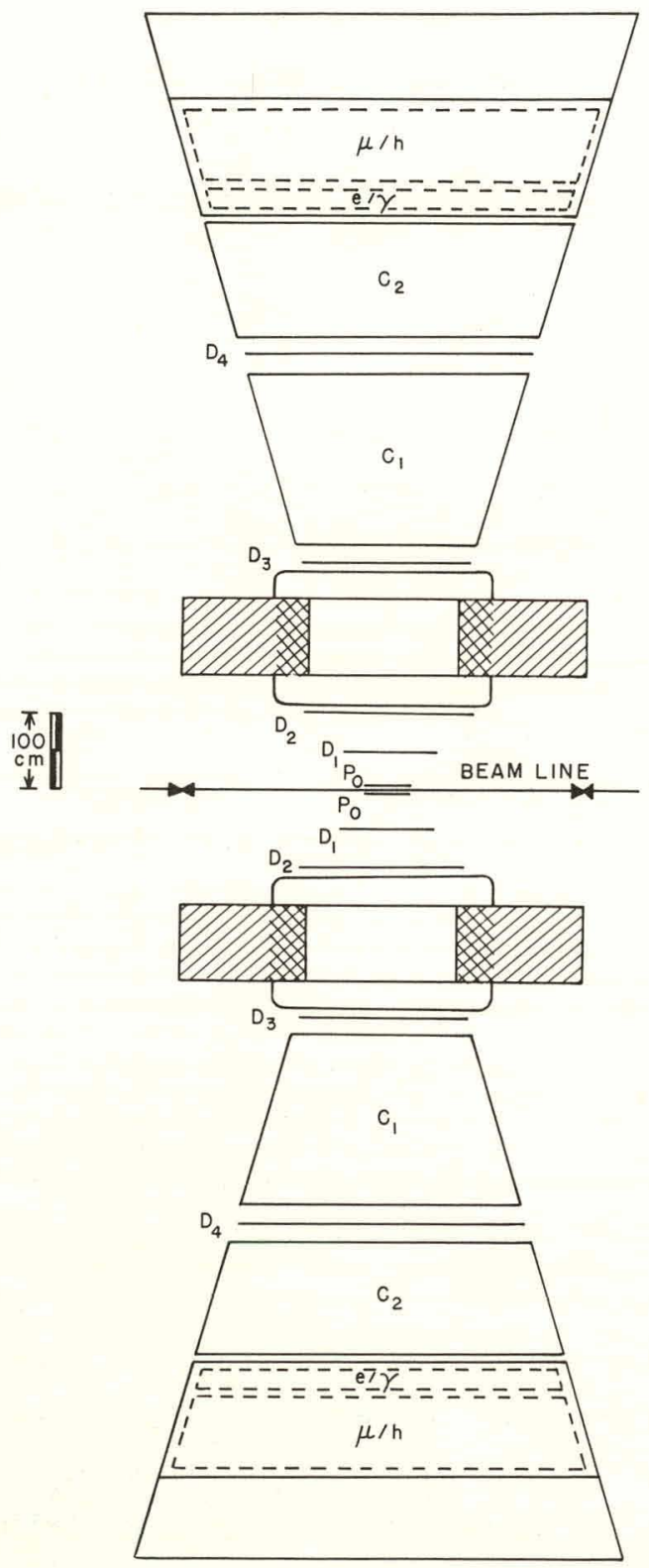

Fig. II.22. Double spectrometer with both arms at $90^{\circ}$ for study of hadrons at large transverse momentum (Peoples 1976). 
stresses particle identification by Cerenkov counters. The objective would be the study of the leading charged particles in a jet. The discovery of charmed particles, in particular the charmed baryon, is a reminder that $\Lambda^{\circ}$ 's and $\mathrm{K}^{\mathrm{O}}$ 's are very important particles to detect.

The hard scattering of a quark in one hadron off a quark in another hadron is expected to give rise to a pair of jets. By including spectrometers on both sides of the beam, correlations between jets can be studied. It seems feasible to study quarkquark scattering by detecting correlated jets with $\mathrm{p}_{\perp}>10 \mathrm{GeV} / \mathrm{c}$. The full luminosity of $10^{33} \mathrm{~cm}^{-2} \mathrm{sec}^{-1}$ can be utilized.

In addition to jets which are produced by quark-quark scattering, there will be jet pairs due to the decay of massive objects such as the $\mathrm{W}^{+}$, Higgs bosons, and $\mathrm{Z}^{\circ} \mathrm{s}$. For example, the $\mathrm{W}^{+}$may decay into a charmed quark and an antistrange quark at an appreciable rate. According to current theory, the quarks do not emerge, and one sees two jets of hadrons carrying integral charge, charm or strangeness. These jets can most easily be detected by using two back-to-back spectrometers which detect both charged and neutra1 particles. One can look to see if there is a peak in the transverse energy distribution. $\mathrm{A} \mathrm{W}^{+}$of $60 \mathrm{GeV}$, for example, would give two jets, each containing $30 \mathrm{GeV}$.

The apparatus, consisting of two magnetic spectrometers oriented perpendicular to the colliding beams, is shown in Fig. II.22. Charged particle trajectories are determined by proportional chambers $\mathrm{P}_{0}^{i}$ and drift chambers $\mathrm{D}_{1}^{i}, \mathrm{D}_{2}^{i}, \mathrm{D}_{3^{i}}$, and $\mathrm{D}_{4^{\circ}}^{i}$. Photon momenta are measured by liquid argon-lead plate calorimeters placed at the end of each spectrometer. Following the argon-lead plate calorimeter there is a stee1-argon calorimeter which, together with the lead, measures the hadronic energy in the jet. The Cerenkov counters $\mathrm{C}_{1}$ and $\mathrm{C}_{2}$ are used to provide particle identification for some of the hadrons. A one meter thick steel wall followed by scintillation 
counters provides for muon identification. Finally each spectrometer could be moved by $\pm 45^{\circ}$ in order to map out jet-jet correlations.

\section{Total Cross Section Measurements}

The measurement of the total cross section is straightforward at conventional accelerators where the transmission method can yield accuracies of $\frac{1}{2} \%$ or better. This simple method is, of course, not available to colliding beam experimenters. Three other methods can, however, provide measurements to about $1 \%$ as described below. [n all cases the variable energy of ISABELLE is particularly valuable for the total cross section measurement. The functional form of the total cross section can be determined from the ISR overlap region of $s=3000 \mathrm{GeV}^{2}$ out to $s=640,000 \mathrm{GeV}^{2}$.

1. In the first method, a $4 \pi$ detector (Chung 1975), shown in Fig. II.23, would be used. The aim is to observe all interactions so that the cross section $\sigma_{\mathrm{T}}$ is determined from

$$
\sigma_{\mathrm{T}}=\mathrm{R}_{\mathrm{T}} / \mathrm{L}
$$

where $R_{T}$ is the total interaction rate and $L$ is the luminosity.

The interaction region is surrounded by detectors as completely as is physically possible. To minimize the ioss of very small angle events, detectors similar to those required for elastic scattering in the Coulomb-nuclear interference region would be used (Marx 1975). They would consist of scintillation counters at $30 \mathrm{~m}$ and $69 \mathrm{~m}$ from the interaction point subtending the angular ranges of $1.3-6.6 \mathrm{mrad}$ and $0.4-1.3$ mrad respectively. At $69 \mathrm{~m}$ the detectors could be nlaced as close as $2.75 \mathrm{~cm}$ to the proton beam which would correspond , $t=0.025(\mathrm{GeV} / \mathrm{c})^{2}$ at $400 \mathrm{GeV}$. Of the order of $2 \mathrm{mb}$ of the total cross section in the forward direction would remain undetected, and that can be corrected by extrapolating the observed events to $0^{\circ}$. This correction should contribute $<\frac{1}{2} \%$ to the error in the total cross section. The limitation of this method is likely to be the absolute knowledge of the luminosity L. Determination of the luminosity to $1 \%$ can be attained (Marx 1974 and Palmer 1975). 


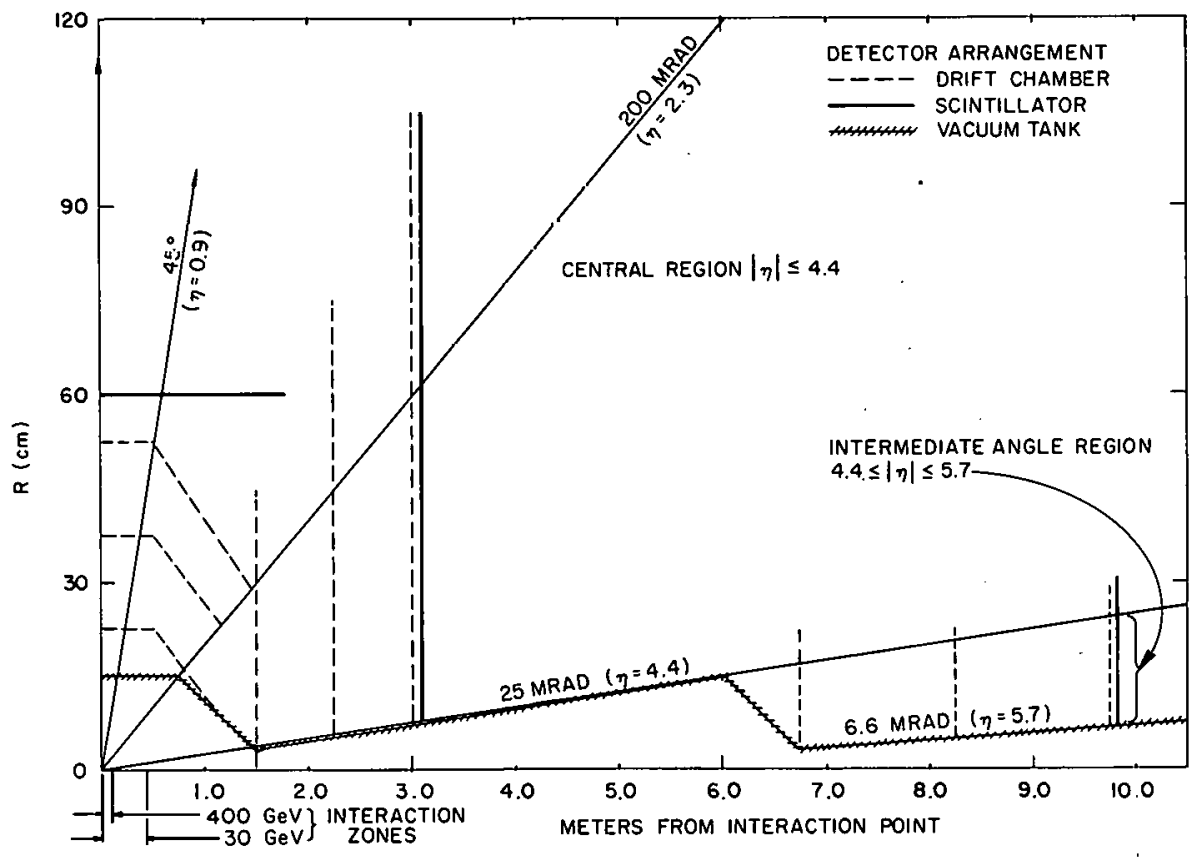

Fig. II.23. Detector arrangement for a " $4 \pi$ experiment" to measure the directions of outgoing particles (Chung 1975). 
The basic detection system is built from drift chamber planes with surrounding scintillation counters. In order to tolerate high luminosity ( $\left.\geqslant 10^{32} \mathrm{~cm}^{-2} \mathrm{sec}^{-1}\right)$ good vertex reconstruction is planned, with the drift chamber wire spacing tailored to the expected particle density distribution as estimated from lower energy experiments at the CERN ISR. Calculation of loss of tracks due to slight losses in solid angle plus additional tracks due to pair production by $\gamma$-rays indicates manageable corrections to the various moments of the multilicity distribution.

It is envisioned that a detector of this type will provide excellent correlation data when used, as at the ISR, in conjunction with spectrometers to select specific particles and ranges of $x$ and $\mathrm{p}_{\perp}$.

In addition a great deal of particle correlation data can be obtained from the $4 \pi$ detector alone.

2. A second method is to extrapolate the differential cross section for elastic scattering to $0^{\circ}$ and use the optical theorem, namely

$$
\begin{aligned}
\sigma_{T} & =\frac{4 \pi}{\mathrm{p}} \operatorname{Im} f(0) \\
& \approx \frac{4 \pi}{\mathrm{p}}\left[\frac{\mathrm{d} \sigma}{\mathrm{d} \Omega}(\theta=0) /\left(1+\rho^{2}\right)\right]^{\frac{1}{2}} \\
& =\frac{4 \pi}{\mathrm{p}}\left\{\left[\mathrm{R}_{\mathrm{E}}(\theta=0) / \mathrm{L}\right] /\left[1+\rho^{2}\right]\right\}^{\frac{1}{2}}
\end{aligned}
$$

where. $R_{E}$ is the elastic rate and $\rho$ the ratio of the real to imaginary Irt of the forward scattering amplitude. This method is a byproduct of the small angle elastic scattering experiment and as such will certainly be done.

3. The third method combines the measurements of both preceding methods. Combining the two expressions gives an expression 
for $\sigma_{\mathrm{T}}$ independent of luminosity $\mathrm{L}$, namely

$$
\sigma_{T}=\frac{R_{E}(\theta=0)}{R_{T}} \times\left(\frac{4 \pi}{p}\right)^{2} \times \frac{1}{1+\rho^{2}}
$$

Measurement of the Real Part of the Forward Scattering Amplitude

An experiment has been designed to measure the Coulomb-nuclear interference using the standard insertion components, requiring only retuning of the quadrupoles to obtain the necessary small angular divergence of the beam (high betatron amplitude function).

The requirements placed on the $\beta$-functions come from the need to measure the elastic scattering to a very small angle with good resolution. Even with ideal detectors the angular spread of the beams contributes significantly to the resolution. Based on a measurement of the Coulomb-nuclear interference from FNAL experiment E69, goals were set on the resolution in $\mathrm{p}_{\perp} \approx \sqrt{ }|\mathrm{t}|$ of $\leqslant 6 \mathrm{MeV} / \mathrm{c} \mathrm{rms}$ with a minimum $|\mathrm{t}|$ of $3 \times 10^{-3}(\mathrm{GeV} / \mathrm{c})^{2}$. (Marx 1975, Chang 1977)

The experimental method is to measure the position of both scattered protons in order to provide an elastic constraint and to use time-of-flight between the two scattered particle detectors to limit events to those originating in the crossing region. The detector location is that labelled "downstream" in Fig. II.24.

The $\beta$-function chosen implies a large beam-beam tune shift which would limit the luminosity to about $10^{30} \mathrm{~cm}^{-2} \mathrm{sec}^{-1}$ as described in Section III.3. If it is assumed that the total acceptance would only be $10 \%$ (solid angle loss, deadtime, packing fraction of detectors, etc.) then one would still be able to collect $10^{6}$ events in 40 hours of running for a luminosity of only $10^{22} \mathrm{~cm}^{2} \mathrm{sec}{ }^{1}$. Thi: is more than an adequate sample to measure $\rho$ to several percent a $L$ one value of $s$.

These measurements would be carried out as a function of $s$ down to $s=3600 \mathrm{GeV}^{2}$ where they. would overlap the ISR experiments. As the momentum of the ISABELLE beams is decreased, the maximum value of $|t|$ which can be reached by the detector described above, 


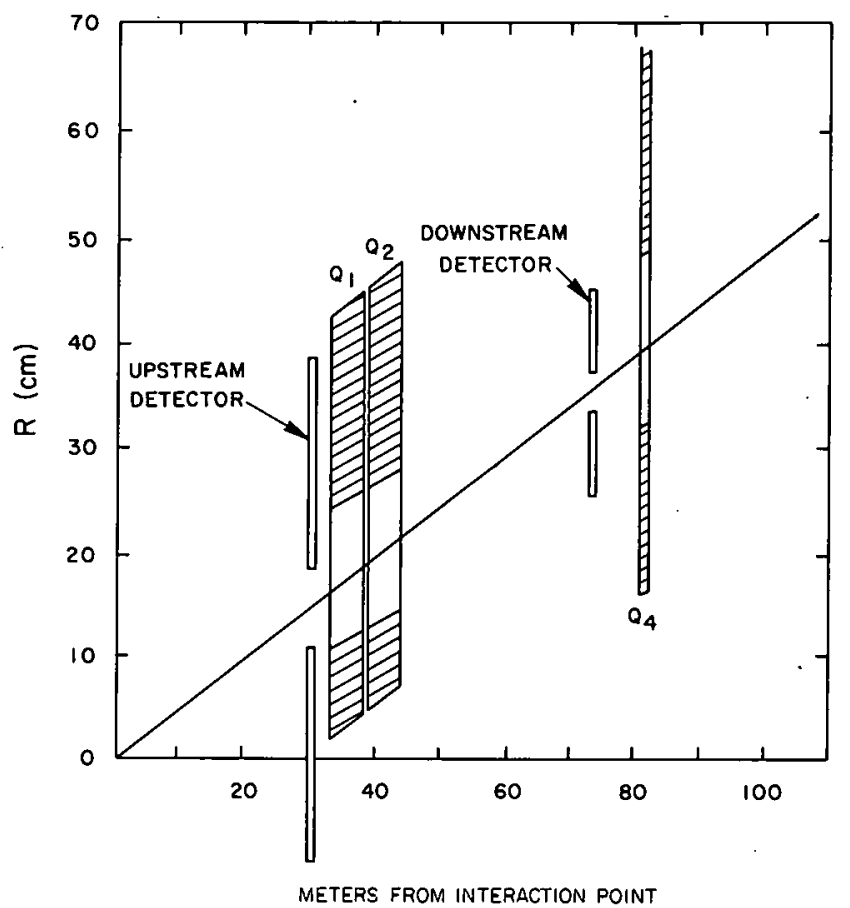

Fig. II.24. Experimental arrangement for the Coulomb-nuclear interference experiment (Mary 1975). 
decreases. The aperture of $\mathrm{Q} 2$ limits $|\mathrm{t}|_{\max }$ to $1.0 \times 10^{-3}(\mathrm{GeV} / \mathrm{c})^{2}$ at $30(\mathrm{GeV} / \mathrm{c})^{2}$ but if a second upstream detector is placed $30 \mathrm{~m}$ from the crossing point and within $3 \mathrm{~cm}$ of the beam, as shown in Fig. II.24, then the coverage in $|t|$ would be extended much higher. For beam momenta between 30 and $400 \mathrm{GeV} / \mathrm{c}$ part of the $|\mathrm{t}|$ range will be covered by the upstream detector, and part will be covered by the downstream detector.

\section{Elastic Cross Section to "Moderate" $t$}

At values of $|t|$ larger than the Coulomb region, the elastic scattering cross section illustrates the clarification revealed by going to high energies, Fig. II. 16.

From the forward diffractive slope, $b(s)$ would be determined from the expression

$$
b(s)=\left.\frac{d}{d|t|}\left(\log \frac{d \sigma(s, t)}{d|t|}\right)\right|_{t=0}
$$

The ISR data indicate a break or change of slope in the forward $p-p$ elastic cross section at about $|t|=0.14(\mathrm{GeV} / \mathrm{c})^{2}$. The sdependence of this phenomenon should be studied at ISABELLE. From 1 to $2 \times 10^{6}$ events would be required at several s values.

The s-dependence of the position and depth of the dip and the height of the secondary maximum should be studied. For a high precision study of these phenomena $10^{4}$ events $/ 0.1$ (Gev/c) ${ }^{2}$ through the range of the dip would be required at each $s$.

The measurement of elastic scattering at small and intermediate values of $|t|$ would be carried out in the apparatus shown in Fig. II.25 (Edelstein 1975, Limon 1976 and Chang 1977). The magnets needed for elastic scattering are $0.8 \times 0.8 \mathrm{~m}^{2}$ aperture with $30 \mathrm{kG}$ m field integral. Large superconducting dipoles are probably required. One could, however, accomplish all but the very high $|t|$ measurements with conventional 48D48 magnets by shimming the gap for the highest $s$ and running at different fields for each $s$. The compensating dipoles would be outside of the apparatus and may be 
any convenient size.

Not enough data exists on elastic scattering for $|t|>5(\mathrm{GeV} / \mathrm{c})^{2}$. ISABELLE presents a unique opportunity to study this region. A sample of $10^{3}$ events/(GeV/c $)^{2}$ at several values of $s$ will be very useful. It should be noted that the apparatus covers the range $1-50 \mathrm{mrad}$, or $0.001<|\mathrm{t}|<2.25(\mathrm{GeV} / \mathrm{c})^{2}$ at $30 \times 30 \mathrm{GeV}$ and $0.111<|\mathrm{t}|<400(\mathrm{GeV} / \mathrm{c})^{2}$ at $400 \times 400 \mathrm{GeV}$. The extent in $|\mathrm{t}|$ to which the measurements can be carried out would depend on the lagnitude of the elastic scattering cross section, the nonelastic vackground and the ultimate resolution of the detectors.

If, during the initial machine operation, the use of the dipoles were not possible, angle-angle correlations would allow the elastic cross section to be measured out to $|t|=1.5(\mathrm{GeV} / \mathrm{c})^{2}$. This would permit the study of the slope of the diffraction peak, including the question of shrinkage and a search for the apparent change of slope at $|\mathrm{t}| \approx 0.14(\mathrm{GeV} / \mathrm{c})^{2}$. This set up would also yield a total cross section measurement via the optical theorem.

The detectors shown in Fig. II. 25 would be a combination of scintillation counters, drift chambers and possibly proportional chambers. Proportional chambers would be incorporated if it is necessary to tolerate very high counting rates at small angles. This would be required, for example, in a study of double diffraction dissociation where the highest luminosities are required. If the system were to be used only for elastic scattering, drift chambers with $1 \mathrm{~cm}$ wire spacing would be adequate. In the forward direction where the rates are high, the cross section is also high, so a sduced luminosity would be suitable. For higher $|t|$ the central Legion of the shambers would be deadened and higher luminosities would be required. The counting rate in the drift chambers would be limited by the chamber which is closest to the interaction region. Since the resolution of a drift chamber varies closely with drift distance, one may use a larger wire spacing in the rear chambers witich are almost twice as far from the interaction region 

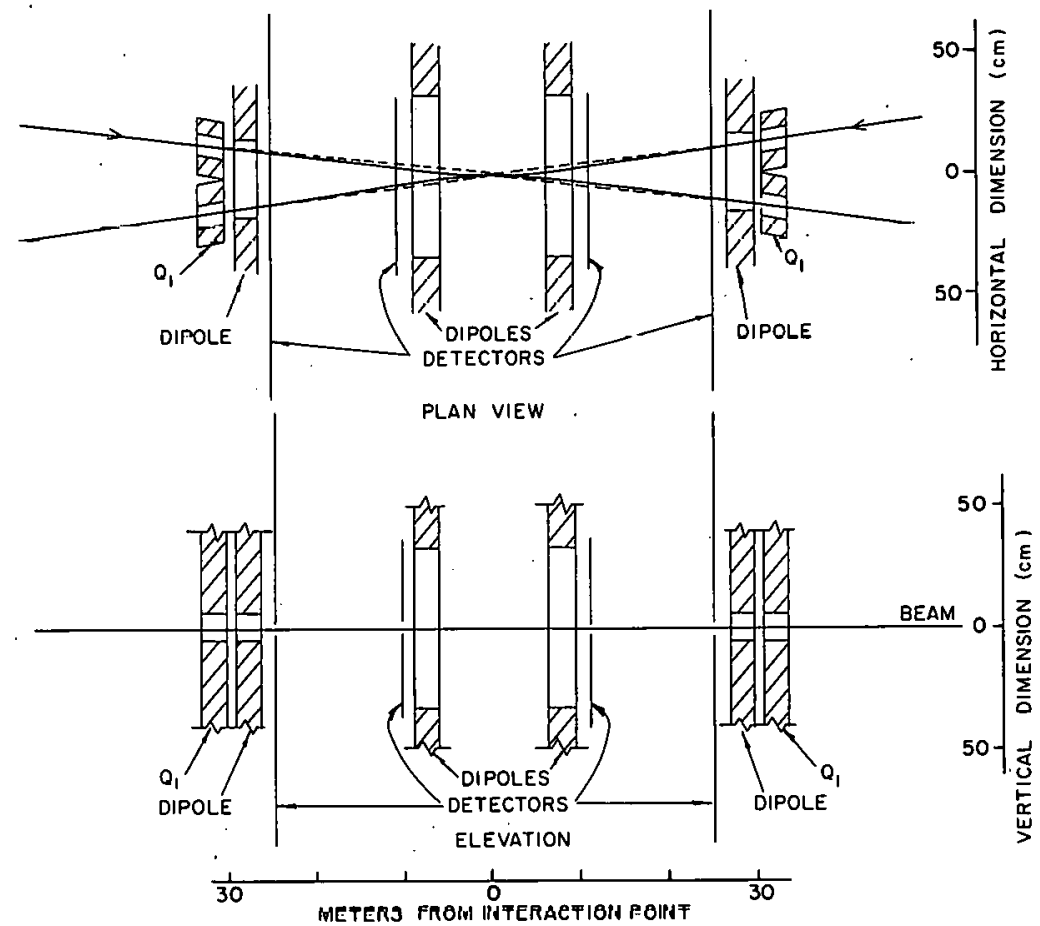

Fig. II.25. Detector arrangement for the measurement of the elastic cross section at moderate $|t|$ (Edelstein 1975). 
as the front chambers. With a $1 \mathrm{~cm}$ wire spacing in the front chambers and a $2 \mathrm{~cm}$ wire spacing in the rear chambers, 1400 wires would be required for the whole system.

\section{Measurement of Inclusive Cross Sections at Sma11 $\mathrm{p}_{1}$}

One of the major challenges to the experimenters at ISABELLE involves the measurement of particle production at very small angles and very high momenta. Since the trajectories followed by these particles are very close to those of primary beam particles, special techniques are required to detect them. However, the great interest in this region, as discussed in Section 2, demands that al1 efforts be made to study it. The capability of varying the two beam energies independently should prove useful in this respect (Peier1s 1975).

A very interesting approach was suggested in the 1975 ISABELLE Summer Study (Chang 1975). Septum magnets close to the vacuum pipe would be used to bend particles to a pivot magnet, which would deflect particles to a fixed spectrometer to measure momenta and identify particles. Such a system would normally be limited to scattering angles larger than about $5 \mathrm{mrad}$ because of the need to bend particles so as to miss the standard quadrupoles. However, the approach suggested was to construct a quadrupole with separated windings, as shown schematically in Fig. II.26a. Here the septa would be placed downstream of Q1 and Q2. With this design, $400 \mathrm{GeV}$ particles can be measured when produced at angles of 1 to $8 \mathrm{mrad}$ for positive particles and 2 to $8 \mathrm{mrad}$ for negative particles. Note that this range $\left(\mathrm{x} \approx 1, \mathrm{p}_{\perp} \geqslant 0.4 \mathrm{GeV} / \mathrm{c}\right.$ at $400 \times 400 \mathrm{GeV}$ ) is irticularly suitable for investigating the triple Regge model and $\sim$, in fact, at lower $P_{\perp}$ than measurements made to date at the CERN ISR.

A more straightforward situation occurs at slightly larger angles ( $\supsetneq 5 \mathrm{mrad}$ ). A viable experiment was described in the 1975 ISABELLE proposa1. In this arrangement septum magnets would be 


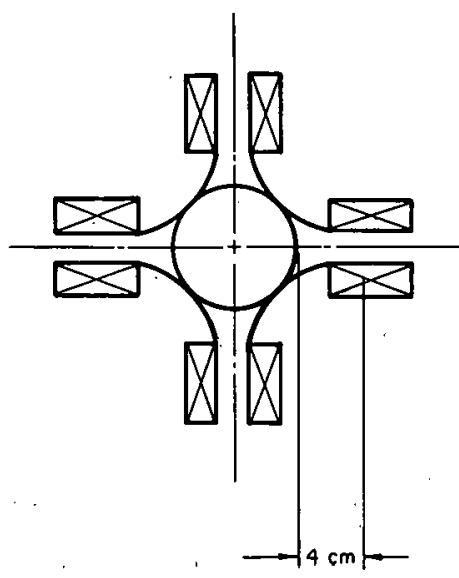

Fig. II.26a. Sketch of quadrupole magnet with slot for secondary particles.

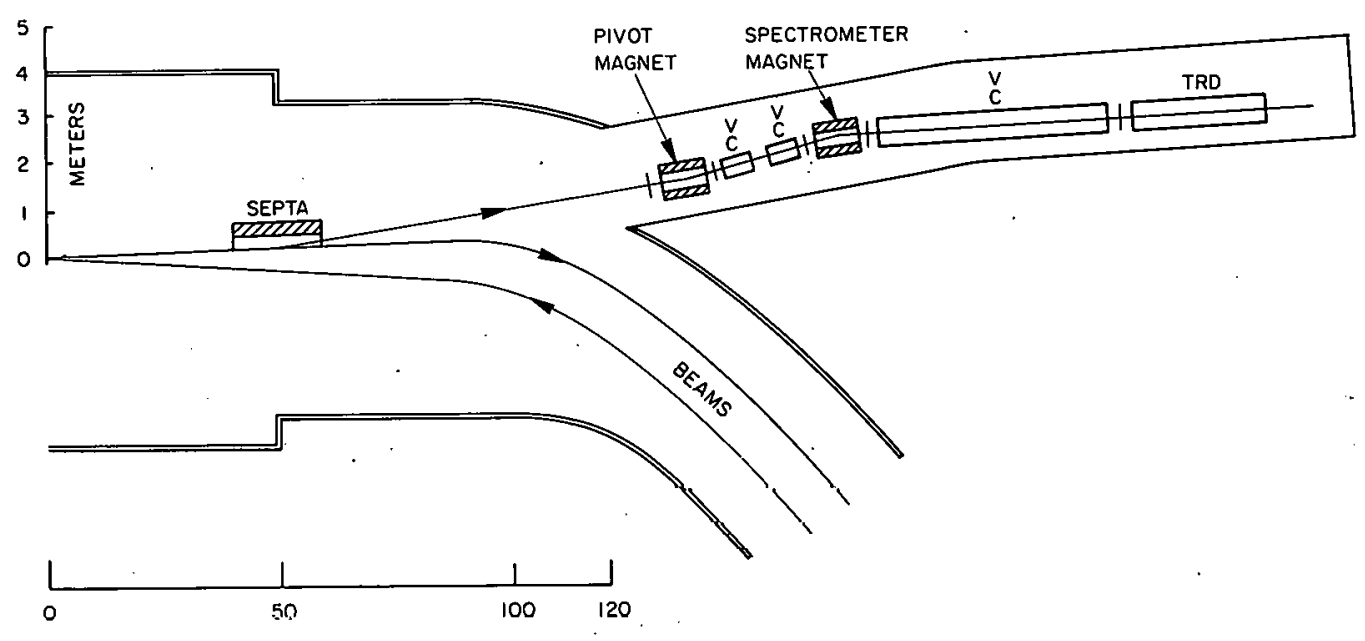

METERS FROM INTERACTION POINT

Fig. II.26b. Experimental layout for small angle spectrometer (Chang 1975). The experimental hall and tunnel are shown schematically. 
placed upstream of the machine quadrupoles and would deflect particles to a pivot magnet, which, in turn would direct the particles to a fixed spectrometer to measure momenta and identify particles.

The spectrometer, shown in Fig. II.26b would be the same for either of the two possibilities considered above. The excitation of the septum magnets and the pivot magnet define the production angle and the momentum of the particles transported to. the spectromter. The pivot magnet and spectrometer magnet are of a simple design. At high momentum multiple scattering is negligible so the momentum resolution of the system is determined by the spatial resolution of the detectors. For example, with $\pm 0.5 \mathrm{~mm}$ resolution we have $\Delta p / p \approx \pm 0.3 \%$. This is easily achieved with proportional wire chambers; better resolution can be obtained with drift chambers, if the experiment demands it. 


\section{DESCRIPTION OF ISABELLE}

1. OVERVIEW

The conceptual design adopted for the intersecting storage accelerator facility, ISABELLE, attempts to provide the greatest performance potential consistent with the constraint of minimizing the construction and operating costs. The basic performance criteria for pp colliding beam machines are the available energy, the collision rate (luminosity), the radiation background (signal-to-noise ratio), adequate space for experimental equipment, and flexibility of the experimental insertion layout. Essential to the ISABELLE design is the application of superconducting magnets.

The concept of colliding beams represents the only practical method of extending the center-of-mass energy by a significant step beyond that available from existing accelerators. ISABELLE, as proposed and described in this section, will provide colliding proton beams, each with a top energy of at least $400 \mathrm{GeV}$. In the center-of-mass system of the colliding particles an energy of $800 \mathrm{GeV}$ will be available which is equivalent to a fixed-target accelerator with $34 \mathrm{U}, \mathrm{UUU} \mathrm{U} \mathrm{GV}$. Clearly, not all signifucant experiments would demand the highest energies and, in tact, it is of considerable importance that a new machine have an energy band which overlaps that of existing machines. ISABELLE has been designed so as to be capable of covering the entire energy range from $60 \mathrm{GeV}$ available at the CERN ISR to $800 \mathrm{GeV}$ in the centerof-mass system with high collision rates.

Luminosity is, in colliding beam devices, the machine parameter which determines the collision rate. The design of ISABELLE foresees luminosities at each crossing region from $10^{32}$ to $10^{33} \mathrm{~cm}^{-2} \mathrm{sec}^{-1}$ over the entire energy range. With the total pp cross section of about $40 \mathrm{mb}$, a luminosity of $10^{33} \mathrm{~cm}^{-2} \mathrm{sec}-1$ 
results in an interaction rate of $40 \mathrm{MHz}$ at each crossing point. The total particle production rate is higher due to the multiplicity per interaction; at the top energy it is estimated to be about 1 billion particles per second.

The proposed facility would consist of two interlaced magnet rings providing the bending and focusing fields for the counter rotating proton beams. The two rings are magnetically uncoupled to allow operation with unequal energies in the rings. the configuration of ISABELLE is essentially a circle broken by six symmetrically placed insertions where the beam lines cross. The circumference of each ring is $3834 \mathrm{~m}$, exactly 4-3/4 times the circumference of the AGS. About one quarter of the circumference is contained in the six insertions, each of $164 \mathrm{~m}$ length. The beams cross horizontally at the center of the straight sections. A line drawing of the ISABELLE rings depicting the geometrical relationship to the AGS injector, the beam transfer lines, the experimental halls and service buildings is shown in Fig. III.1. This location was chosen in order to take advantage of the existing external proton beam line to the 7 foot bubble chamber. The terrain to the north of the AGS is adequate in size to accommodate the ISABELLE rings with sufficient distances to the site boundaries and the North exit road of the Laboratory.

The two magnet rings are located side by side in a common tunne1. The ring separation is $94 \mathrm{~cm}$ from magnet center to magnet center. The cross section of the tunnel is shown in Figure III.2. The circular tunnel structure has a width of $6 \mathrm{~m}$ and is $3.1 \mathrm{~m}$ high. The tunnel will be covered with $4 \mathrm{~m}$ of - Ind for radiation shielding. In addition, the facility is placed far enough from the site boundaries to permit adequate muon shielding. 


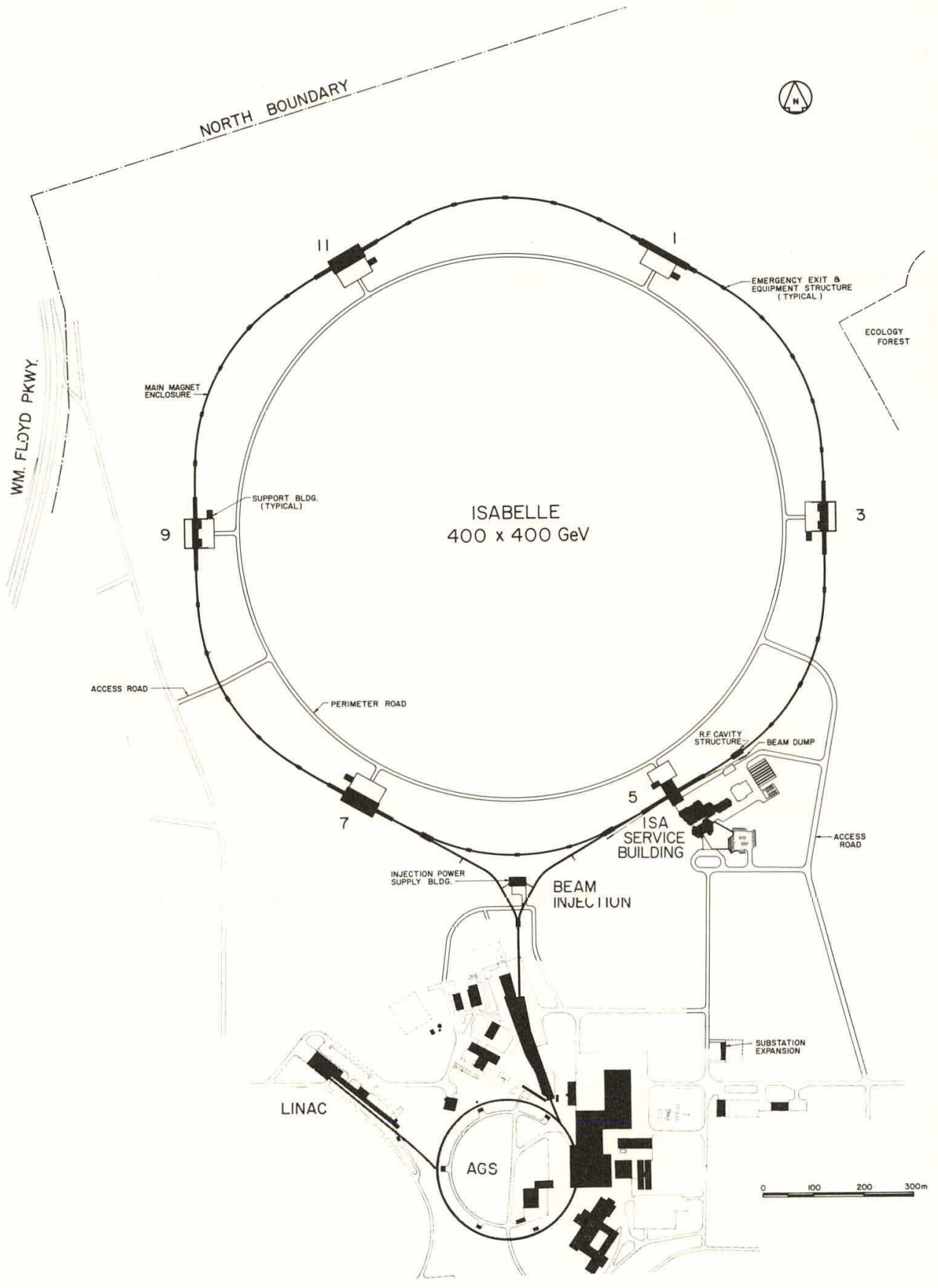

Fig. III.1. Plan view of the ISABELLE ring showing the AGS as the injector. The experimental halls are designated by their "hand of clock" position around the ring. 


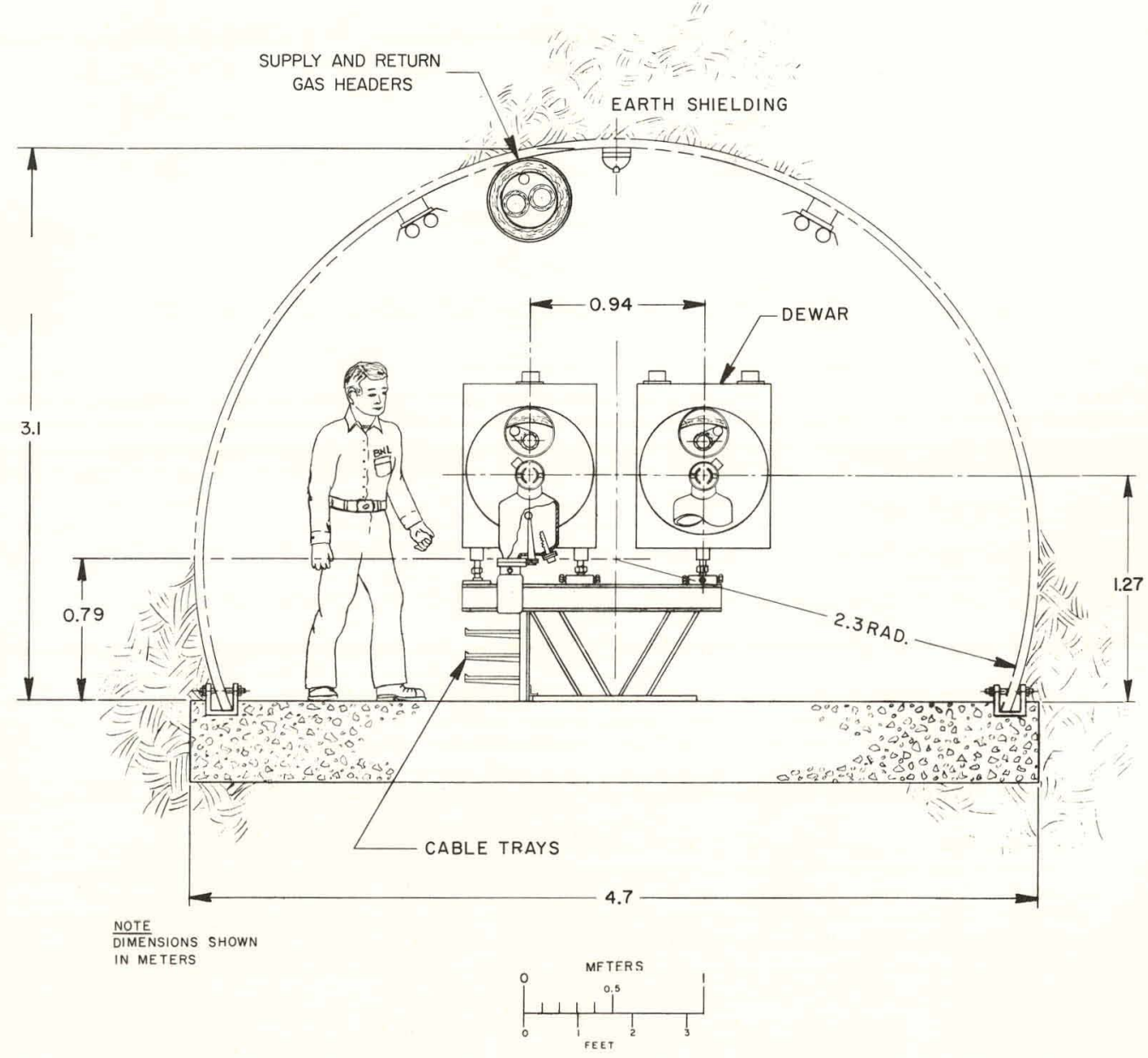

Fig. III.2. Cross section of the ISABELLE tunne1 (dimensions in meters). 
Performing experiments on a storage ring must take into account the close interrelationship between the design of each experiment and the constraints imposed by the machine. of paramount concern to the experimenter is the length of the free space around the crossing point available for the particle detecting apparatus. The standard insertions at ISABELLE provide $60 \mathrm{~m}$ free space between the nearest quadrupoles. Inherent in the experimental insertion design is a considerable flexibility in changing the beam optics in order to accommodate different experimental requirements. The concept of using only matched insertions in a separated function lattice provides the possibility of varying the beta-function at the crossing point. However, it was thought prudent to retain initially the option of operating with quasi-six-fold symmetry. The standard insertion is characterized by a vertical $\beta^{*}=7.5 \mathrm{~m}$ at the crossing point. The low-beta insertion with $\beta^{*}=2 \mathrm{~m}$, allowing a doubling of luminosity, is obtained by modifying the focusing strength of the insertion quadrupoles without geometrical changes. A similar approach could be used in a high-beta insertion to achieve an angular resolution of about $10 \mathrm{mad}$. The highest luminosity will be achieved by reducing the crossing angle through the addition of four dipoles, common to both rings, in the low-beta insertion. A similar magnet configuration may be used to increase the crossing angle in the sma11-diamond insertion.

The AGS will serve as injector of $30 \mathrm{GeV}$ protons for ISABELLE. In this mode of operation, the AGS would be tuned for the greatest beam brightness, which is achieved by operating at lower than peak intensity. In order to accumulate the design current of $8 \mathrm{~A}$ in each ring, about 300 AGS pulses of $2.7 \times 10^{12}$ protons will be stacked in momentum space. Filling of one ring is estimated to take a minimum of 15 minutes. Prior to accelefation 
the stacked beam will be rebunched on the third harmonic. The acceleration cycle will take about 4 minutes. The required peak rf voltage of $48 \mathrm{kV}$ per ring will be provided by four ferriteloaded cavities. After acceleration, beams will nomally be debunched for collisions. At the end of the useful lifetime of the stored beams, which is expected to be typically one day, the beams will be extracted by means of a fast protective beam dump system into an external beam dump. An internal absorber will be available for redundancy to provide added. protection for ring hardware and nagnets .

The ISABELLE magnet system will be superconducting because of the enhanced performance capability and reduced electric power consumption made possible by this approach. The regular lattice structure assumes dipole magnets which are 4-3/4 $\mathrm{m}$ in length and operate at about $50 \mathrm{kG}$ to achieve $400 \mathrm{GeV}$ beam energy. The regular quadrupole magnets are about $1-2 / 3 \mathrm{~m}$ in length, and would operate with a gradient of $6.1 \mathrm{kG} / \mathrm{cm}$. There will be a total of 732 dipoles and 352 quadrupoles in the two rings. The magnet design is based on a single-layer cosine coil arrangement with a cold iron core to provide the mechanical support required for stable operation. The superconducting coils are wound with a conductor braided from niobium-titanium wires which carries about $4 \mathrm{kA}$ at $50 \mathrm{kG}$. The design operating temperature for the superconducting magnets is $3.8 \mathrm{~K}$. Cooking of the magnets is achieved by forced circulation of supercritical helium. Supercritical helium is a single-phase gas with high density and extremely low viscosity, which make it an excellent heat transfer medium. le estimated steady-state heat load at $3.8 \mathrm{~K}$ is $13 \mathrm{~kW}$, which will be removed by a single $19 \mathrm{~kW}$ refrigerator. Cooldown of the entire magnet system is expected to take about 2 weeks. 
The achievement of ultrahigh-vacuum conditions in the beam tube is essential to maintaining the long lifetime, low beam losses, and sma11 associated radiation background which is required in a colliding beam device. The design calls for a hydrogen pressure of less than $3 \times 10^{-11}$ Torr in the lattice sections of the ring and lower in the experimental insertions. The $8.8 \mathrm{~cm}$ i.d. stainless steel vacuum chamber will be at room temperature. Proper surface treatments prior to installation together with the possibility for a $300^{\circ} \mathrm{C}$ in situ bakeout assures that the vacuum specifications will be met.

A more detailed discussion of the accelerator subsystems is covered in the following sections. A list of the main parameters of ISABELLE is given in the Appendix. 
The separated function lattice structure initially will have quasi-six-fold symmetry. The main portion of the bending is done in 54 regular FODO cells, 9 per sextant, with 6 bending magnets per cell. The magnet layout for a half-sextant is sketched in Fig. III.3. A small part of the bending is performed by the $1 \frac{1}{2}$ modified cells on either side of the insertion. These cells ("dispersion matching sections") are designed to bring the two beams together at the crossing point with a crossing angle of $9.4 \mathrm{mrad}$, and to bring the dispersion function to zero in the central part of the insertion. This is accomplished by having only three regular bending magnets in these $1 \frac{1}{2}$ modified cells (instead of nine), plus one shorter bending magnet, $2.5 \mathrm{~m}$ long, in the outer arc. The matching cells have the same focusing structure as the regular cells except for a slight increase in length $(0.5 \mathrm{~m})$. A list of the ISABELLE lattice parameters is given in Table I.

The spacing of the magnet elements in the insertions, including the adjacent moditied bending cells, are shown in Fig. III.4. Between the collision points and the matching cells two quadrupole doublets Q1, Q2, Q3 and Q4 match the beam optics to that of the regular cells with amplitude functions $\beta_{h}^{*}=30 \mathrm{~m}$, $\beta_{\mathrm{v}}^{*}=7.5 \mathrm{~m}$ at the crossing point. The Q1-Q2 pairs in the two Intersecting beams are staggered in location, with $30 \mathrm{~m}$ from the crossing point to $\mathrm{Q} 1$ in the outer arc and $33 \mathrm{~m}$ in the inner arc; following these doublets there are additional free spaces of 42 and $36 \mathrm{~m}$, respectively, between quadrupole Q2 and Q3, which are Ilso available to some extent for experimental apparatus. The yuadrupole Q3, furthermore, is redundant in the standard configuration and has been left out (lengthening the free spaces to 46 and $40 \mathrm{~m}$ ), but it is required in the "1ow beta" and "high beta" configurations discussed below. Some of these straight sections will also be used for equipment needed for various machine functions; in particular, injection, ejection, rf systems and special beam diagnostics. The amplitude and hortzontal dispersion 


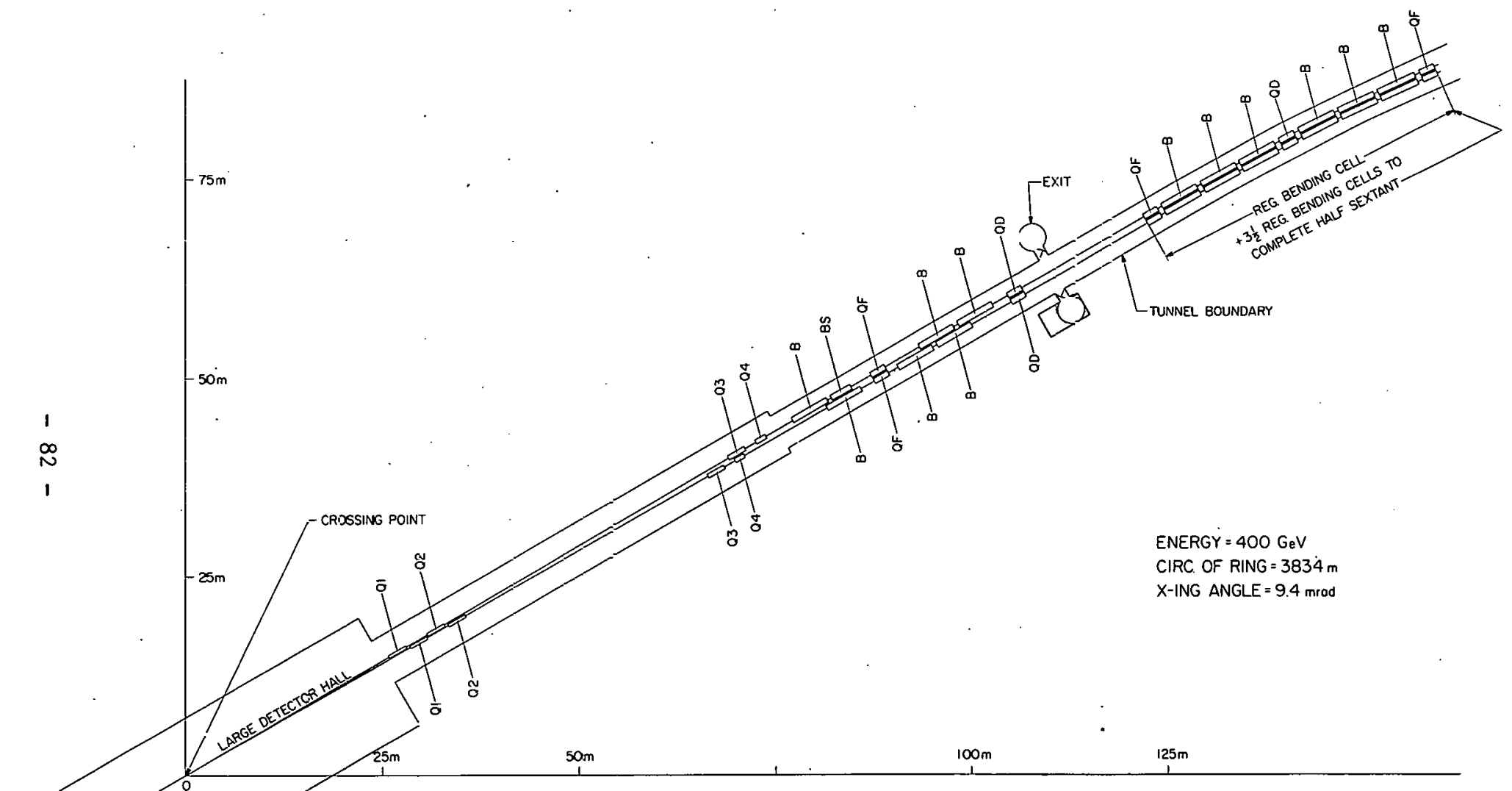

Fig. III.3. Zortion of the ISABELLE half sextant, showing the insertion magnets and one regular lattice bending cel-. An experimental rall and the stairwells for tunnel acces:s are shown. Additiona space is provided for power supplies and other equipment. 


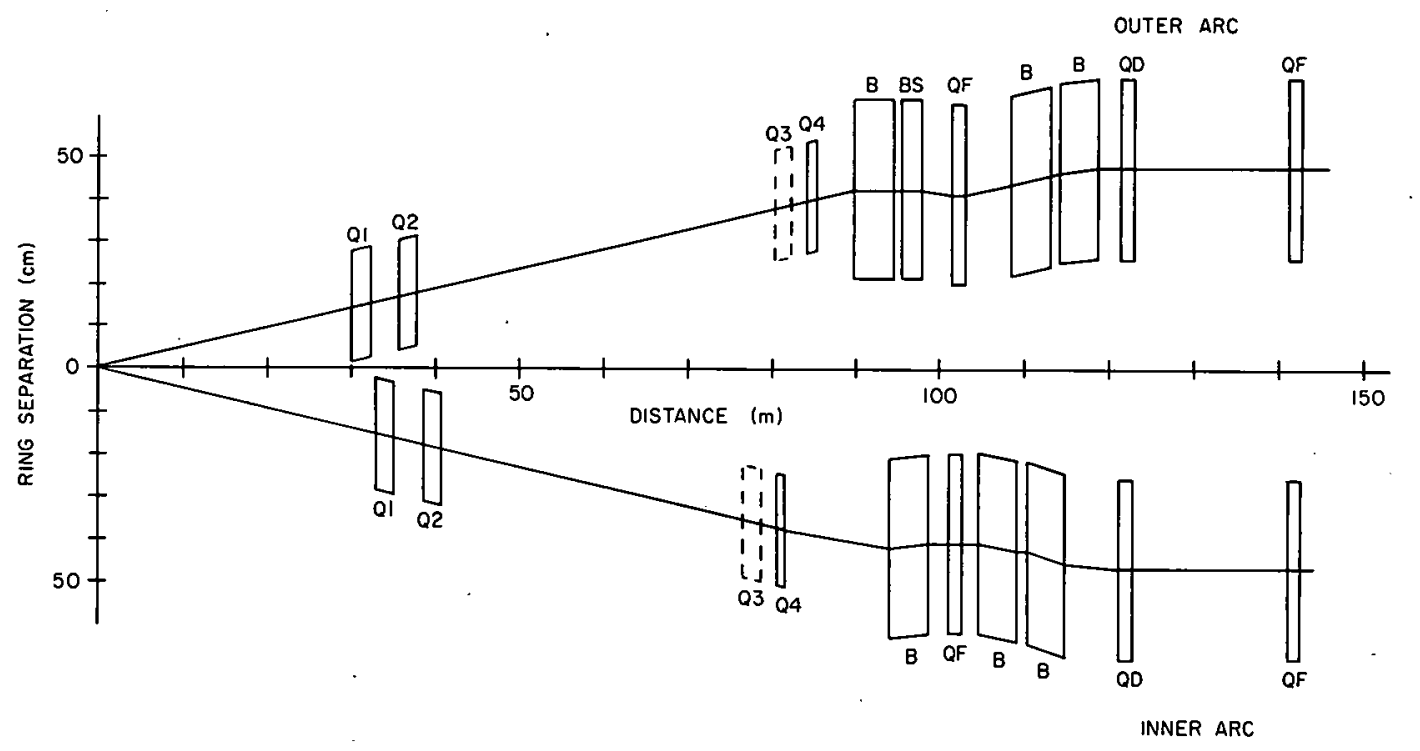

Fig. III.4. Magnet elements for the standard insertion shown in their relative positions. 
Dipole magnetic field (400 GeV)

Quadrupole gradient (cel1s, $400 \mathrm{GeV}$ )

Circumference $\left(4-3 / 4 C_{\text {AGS }}\right)$

Regular cells (no. $\times$ length)

Dispersion matching sections (no. $X$ length)

Insertions (no. $x$ length)

Horizontal beam separation in arcs

Minimum beam separation (QFI-QFO)

Regular cell length - inner arc

Regular cell length - outer arc

Average radius of curved arc

Magnetic radius of curvature

Average machine radius

Tune $\left(\nu_{h}=v_{v}\right)$

Working line tune spread $\left(\Delta \nu_{h}=\Delta \nu_{v}\right)$

Transition energy, $\gamma_{t r}$

Frequency slip factor, $\eta$ (injection)

Horizontal amplitude function, $\operatorname{cell}\left(\beta_{\max }, \beta_{\min }\right)$

Vertical amplitude function, $\operatorname{cell}\left(\beta_{\max }, \beta_{\min }\right)$

Dispersion function, $\operatorname{cell}\left(\mathrm{X}_{\mathrm{p}, \max }, \mathrm{X}_{\mathrm{p}, \min }\right)$

Phase Advance, cell $(h, v)$

Natural lattice linear chromaticity

(no sextupole correction)

Operating chromaticity

$\left(x_{h}=X_{v}\right.$, full coasting stack, $\left.29.4 \mathrm{GeV}\right)$

$$
\begin{gathered}
49.85 \mathrm{kG} \\
6.06 \mathrm{kG} / \mathrm{cm} \\
3833.8 \mathrm{~m} \\
\times 39.5 \mathrm{~m} \\
\times 59.6 \mathrm{~m} \\
\times 164.3 \mathrm{~m} \\
0.94 \mathrm{~m} \\
0.82 \mathrm{~m} \\
39.45 \mathrm{~m} \\
39.55 \mathrm{~m} \\
380.6 \mathrm{~m} \\
267.7 \mathrm{~m} \\
610.1 \mathrm{~m} \\
22.62 \\
\approx 0.02
\end{gathered}
$$$$
19.11
$$$$
1.72 \times 10^{-3}
$$

$67.1,11.1 \mathrm{~m}$ $67.3,11.3 \mathrm{~m}$ $2.64,1.26 \mathrm{~m}$ $92.9 \cdot 91.3 \mathrm{deg}$ $-33$ $+2$

functions for the standard and low-beta configuration are shown in Fig. III.5.

The horizontal separation of the beams before being brought together is $94 \mathrm{~cm}$, providing more than sufficient space to accommodate separate dewars for individual inner and outer arc magnets. The distance between the two beams is reduced to $82 \mathrm{~cm}$ at the location of quadrupoles QFi-QFO in the matching sections. This is 


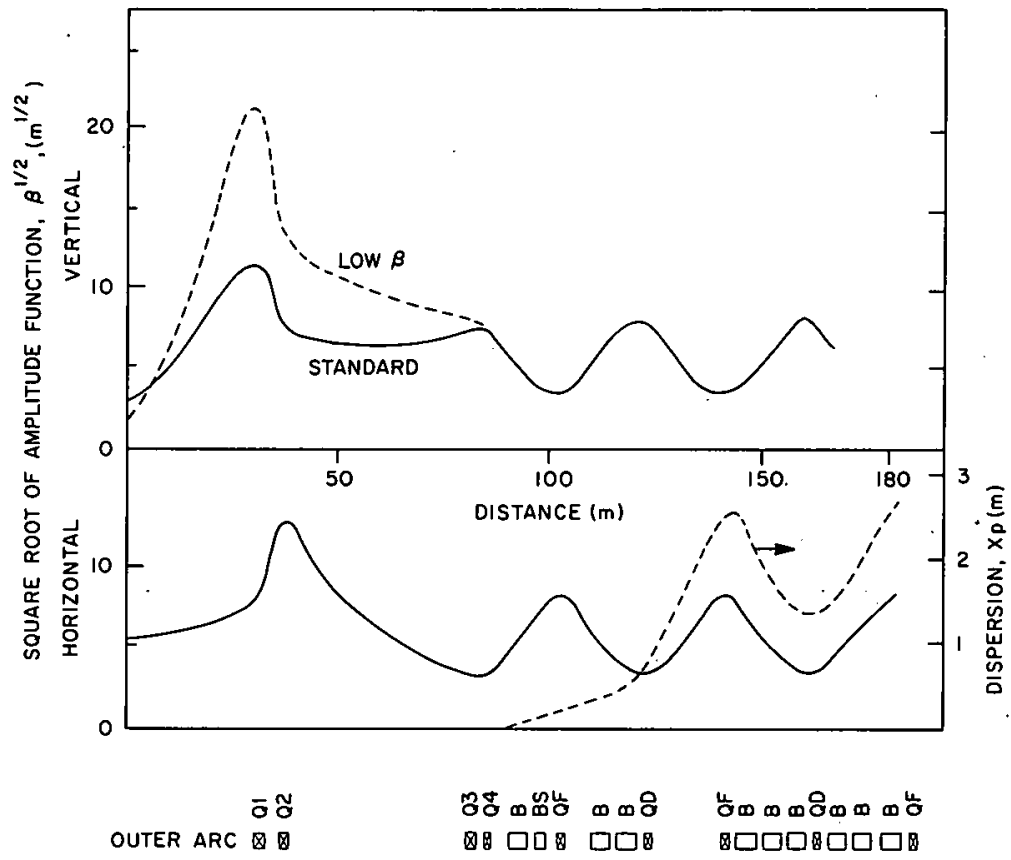
Fig. III.5. ISABELLE amplitude and dispersion functions $\left(\beta_{h}, \beta_{v}\right.$ and $\left.x_{p}\right)$
for the outer arc of the rings. The functions for the inner arc are essentially the same. 
the minimum distance encountered by "paralle1" magnets as the beams are made to converge towards each other. The Q1-Q2 quadrupole locations are staggered so that the quadrupole units of the inner arc fit between those of the outer arc, and vice versa (Fig. III.4). Therefore, there is enough space between the inner and outer beam as long as these quadrupoles and their vessels have a diameter of not more than about $48 \mathrm{~cm}$. The other magnets in the insertion are in vessels of $75 \mathrm{~cm}$ diameter, the same as the regular cell magnets, which leaves adequate space for the separate beams.

The quadrupoles $\mathrm{Q} 1, \mathrm{Q} 2$ and $\mathrm{Q} 4$ a11 have the same gradient $(6.06 \mathrm{kG} / \mathrm{cm})$ as those in the regular lattice cell so that they can be powered in series with the main lattice magnets, except for trimming adjustments as discussed in section III.5.

The betatron matching can only be achieved at one central momentum. For off-momentum particles; a mismatch results (Month 1972, Garren 1975, Chasman 1975, Autin 1975, Donald 1975, and Edwards 1975a). The primary effect on off-momentum particles is the tune shift due to the natural lattice chromaticity. However, by introducing higher order multipoles into the magnetic field, the tune variation with momentum can be removed. Since for transverse beam stability there must be a residual tune variation with momentum, i.e., a working line, these multipoles provide the necessary control of its shape (Month 1973, Zotter 1972). On the other hand, the $\beta$-function mismatch is not eliminated. In the standard insertion configuration, where the maximum values of the amplitude or structure function are $\beta_{\mathrm{v}, \text { max }} \approx 150 \mathrm{~m}$ for vertical and $\beta_{\mathrm{h}, \max } \approx 180 \mathrm{~m}$ for horizontal dimensions respectively, we plot in Fig. III.6a the variation of $\beta$ with momentum at the crossing point. This variation is typical of the maximum variation around the ring. 


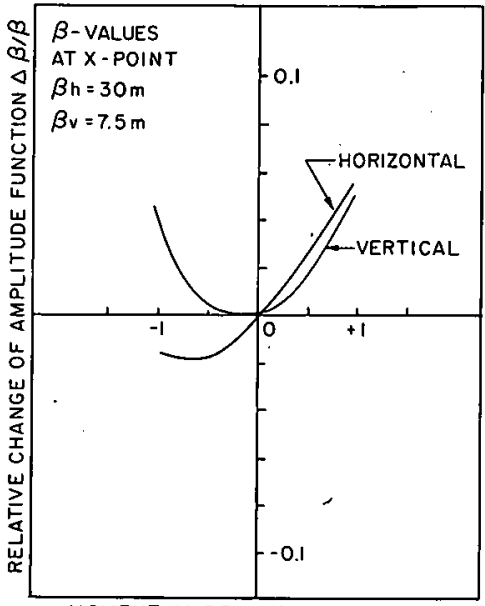

MOMENTUM DEVIATION $\triangle p / p(\%)$

Fig. III.6a. $\beta$ variation across the momentum aperture at the crossing point for the standard lattice configuration.

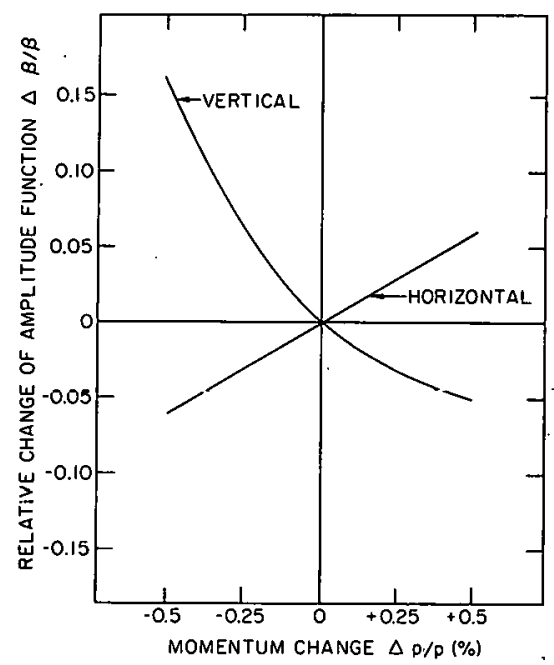

Fig. III.6b. B variation across the momentum aperture at the crossing point for the high luminosity configuration. 
The particular focusing arrangement in the insertions allows changeovers from the standard into the low- $\beta$ and high-beta configuration solely by quadrupole tuning without the need for quadrupole movements. The required quadrupole settings are 1isted in Table 2 and the resulting amplitude functions are shown in Fig. III.5. In the low- $\beta$ configuration, $a \beta_{v}^{*}=2 \mathrm{~m}$ can be achieved resulting in a maximum vertical $\beta$-value of $550 \mathrm{~m}$ at the insertion quadrupoles. In the high- $\beta$ configuratoon, a $\beta_{h}^{*}=\beta_{v}^{*}=100 \mathrm{~m}$ is obtained. To maintain the same overall tunes in the low and high-beta configurations as in the standard one, the regular cell quadrupoles have t، be retuned slightly, as shown in Table 2 .

Table 2. Quadrupole Settings for Insertion Tuning

\begin{tabular}{|c|c|c|c|c|}
\hline \multirow[b]{2}{*}{ Element } & \multicolumn{3}{|c|}{ Gradient $(\mathrm{kG} / \mathrm{cm})$} & \multirow{2}{*}{$\begin{array}{l}\text { Magnetic } \\
\text { length (m) }\end{array}$} \\
\hline & Standard & $10 w-\beta$ & high-B & \\
\hline QF (cel1 quad) & 6.06 & 6.05 & 6.11 & 1.66 \\
\hline QD (cell quad) & -6.06 & -6.08 & -6.11 & 1.64 \\
\hline $\begin{array}{l}\text { Q4 (outer arc) } \\
\text { Q4 (inner arc) }\end{array}$ & $\begin{array}{l}-6.06 \\
-6.06\end{array}$ & $\begin{array}{l}-1.66 \\
+0.22\end{array}$ & $\begin{array}{l}-6.67 \\
-5.56\end{array}$ & $\begin{array}{l}1.15 \\
0.93\end{array}$ \\
\hline $\begin{array}{l}\text { Q3 (outer arc) } \\
\text { Q3 (inner arc) }\end{array}$ & $\begin{array}{l}0 \\
0\end{array}$ & $\begin{array}{l}-0.90 \\
-1.46\end{array}$ & $\begin{array}{l}-0.87 \\
-0.42\end{array}$ & $\begin{array}{l}2.00 \\
2.00\end{array}$ \\
\hline $\begin{array}{l}\text { Q2 (outer arc) } \\
\text { Q2 (inner arc) }\end{array}$ & $\begin{array}{l}6.06 \\
6.06\end{array}$ & $\begin{array}{l}6.27 \\
6.30\end{array}$ & $\begin{array}{l}4.74 \\
4.40\end{array}$ & $\begin{array}{l}2.09 \\
2.12\end{array}$ \\
\hline $\begin{array}{l}\text { Q1 (outer arc) } \\
\text { Q1 (inner arc) }\end{array}$ & $\begin{array}{l}-6.06 \\
-6.06\end{array}$ & $\begin{array}{l}-6.21 \\
-6.39\end{array}$ & $\begin{array}{l}-4.28 \\
-3.56\end{array}$ & $\begin{array}{l}2.24 \\
2.13\end{array}$ \\
\hline
\end{tabular}

The impact of breaking the six-fold symmetry by the inclusion of low- $\beta$ insertions can be seen in plots of the vertical $\beta$-function with momentum. The momentum variation of the horizontal $\beta$-functior is not significantly altered. The effect of inserting a high- $\beta$ configuration is small and can be essentially ignored. The impact of breaking symmetry on the $\beta$-function mismatch can be seen in Fig. III.6, where we contrast the two cases of standard, and low- $\beta$ insertion (Garren 1975). 
The variation of $\beta$ versus momentum shown in Fig. III. 6 was calculated with the central momentum chromaticity (both vertical and horizontal) set to +2 with two independent sets of sextupoles, one family located in the bending magnets nearest to the horizontally focusing quadrupoles, the other in those nearest to the vertically focusing quadrupoles. At present, there are no plans to introduce a more elaborate sextupole system to attempt to reduce this $\beta$-variation, although in principle this could be achieved (Wiedemann, 1976). The primary reason is that the increase in the izimuthal harmonic structure of the sextupole distribution would cause an increase in the strength of the $3 \mathrm{rd}$ order resonances which could result in growth of the beam size.

ISABELLE is designed to allow operation with a symmetry in the focusing structure of almost 6 . The symmetry breaking which results from the difference in inner and outer arc configurations is insignificant. The working line will consist of a straight line along the main diagonal in the $\left(\nu_{h}, \nu_{v}\right)$ plane of length $\Delta \nu \approx 0.02$ to provide sufficient Landau damping of transverse resistive wall instabilities and will be centered around $\nu_{h}=\nu_{v} \approx 22.62$. With our choice of tunes, structure or strong resonances up to very high order can be avoided; the closest is the 4 th order structure resonance at 22.5. In particular, the operating region is well removed from all the structure resonances excited by the chromaticity correcting sextupoles. Since the beam-beam interaction in the standard lattice configuration also has a symmetry of 6 , the low order structure resonances (i॰e., the even-ordered resonances) are again well removed, the losest being the 4 th order resonance at $\nu=22.5$. 


\section{PERFORMANCE}

The basic ISABELLE design draws extensively upon the experience acquired at the only existing set of $\mathrm{p}-\mathrm{p}$ storage rings, the CERN ISR. With this information, ISABELLE has been designed to have an improved performance potential. (1) By accelerating the protons, ISABELLE provides pp collisions over a large range of center-of-mass energies, $60 \mathrm{GeV}$ to $800 \mathrm{GeV}$, as well as allowing independent energy settings in each beam from $30 \mathrm{GeV}$ to $400 \mathrm{GeV}$. (2) With its small crossing angle and low- $\beta$ characteristics, ISABELLE can reach luminosities higher by a factor of 20 ISR, about $10^{33} \mathrm{~cm}^{-2} \mathrm{sec}^{-1}$ with substantially smaller circulating beam currents, $I=8 \mathrm{~A}$. (3) 'l'he use of matched Insercions allows the design of a stable lattice (stable over a sufficient momentum aperture) which has a long magnet-free straight section around the central col1ision point. In the standard ISABELLE insertion design, $\pm 30 \mathrm{~m}$ can be used for experimental detection and measurement apparatus.

The performance characteristics of ISABELLE are given in Table 3 (Hahn 1977). Starting from the standard quadrupole configuration giving a luminosity at high energy of $2.3 \times 10^{32} \mathrm{~cm}^{-2} \mathrm{sec}^{-1}$ addition of a second quadrupole Q3 to the vertically focusing Q4 at the entrance to the insertion leads straightforwardly to the low- $\bar{\beta}$ configuration, with a luminosity of $4.5 \times 10^{.32} \mathrm{~cm}^{-2} \mathrm{sec}^{-1}$. To reach the design luminosity of $10^{33} \mathrm{~cm}^{-2} \mathrm{sec}^{-1}$, large aperture bending magnets are required to reduce the crossing angle from its standard value of $9.4 \mathrm{mrad}$ to $4 \mathrm{mrad}$. The space needed for these bending magnets reduces the amount of magnet-free region around the collision point from $\pm 30 \mathrm{~m}$ to about $\pm 15 \mathrm{~m}$. The required aperture in these bending magnets depends on just how much free space we are willing to sacrifice and could be as large as $25-30 \mathrm{~cm}$. The performance characteristics of a "sma11-diamond" insertion are also listed in Table 3. In this case, large aperture bending magnets are required to increase the crossing angle to $35 \mathrm{mrad}$. 
TABLE 3. ISABELLE Performance

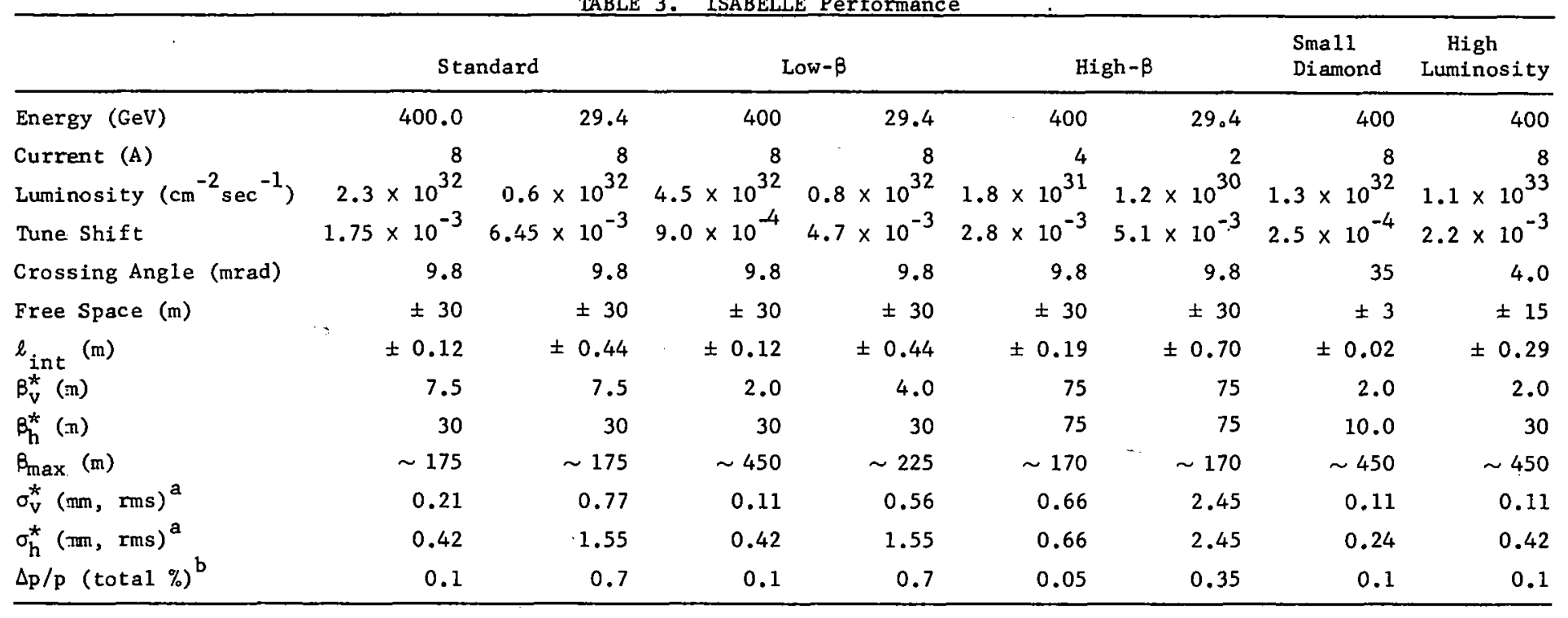

${ }^{a}$ The normalized emittances (enclosing $95 \%$ of the particles in phase space) are: $E_{v}=E_{h}=15 \pi \times 10^{-6}$ rad-m.

$\mathrm{b}_{\mathrm{To}}$ obtain the value of $\Delta \mathrm{p} / \mathrm{p}$ at $400 \mathrm{GeV}$ a factor of $2 \mathrm{dilution}$ was assumed to take into account rebunching, acceleration and debunching of the ISABELLE beam. 
As in the high luminosity setup, a tradeoff between crossing angle and free space is required to accomplish this. To increase the crossing angle to $35 \mathrm{mrad}$ results in a reduction of the free space around the collision point to $\pm 3 \mathrm{~m}$. Possible bending configurations giving the required $4 \mathrm{mrad}$ and $35 \mathrm{mrad}$ crossing angles for the high luminosity and small diamond arrangements respectively are illustrated in Fig. III. 7.

ISABELLE performance is predicated on the choice of crossing angle and stack current. The significance of these parameters is reflected in the beam-beam tune shift which they produce under various conditions. Since the beam-beam tune shift is a measure of the strength of the beam-beam interaction and is positively correlated with radiation background in the experimental areas, it was deliberately chosen to have small value. For example, the low- $\beta$ configuration has $\Delta \nu \approx 9 \times 10^{-4}$, only about a factor of 3 larger than at the ISR. However, the beam-beam tune shift alone does not sufficiently describe the interaction of the two beams. Rather, it is the difficulty of beam alignment at the collision point which is expected to be the dominant reason for beam-beam resonance excitation. In ISABELLE, just as at the ISR, it is the odd-ordered nonlinear resonances, excited by random errors in the closed orbit positioning at the collision point which are expected to be the main sources of beam loss (Bryant 1974 and Henrichsen 1974). It is expected, again as at the ISR, that this beam loss will be the dominant source of experimental background. It is estimated that, at the tune shift levels proposed for ISABELLE, acceptable beam loss rates will result with orbit control at the collision points of about $0.03 \mathrm{~mm}$. With the present design, however, greater orbit precision could allow a larger tune shift and thus a luminosity larger than the design value.

The ISABELLE operational procedure maintains the standard six-fold symmetry during stacking and acceleration. After an initial phase where collisions take place with the standard six- 

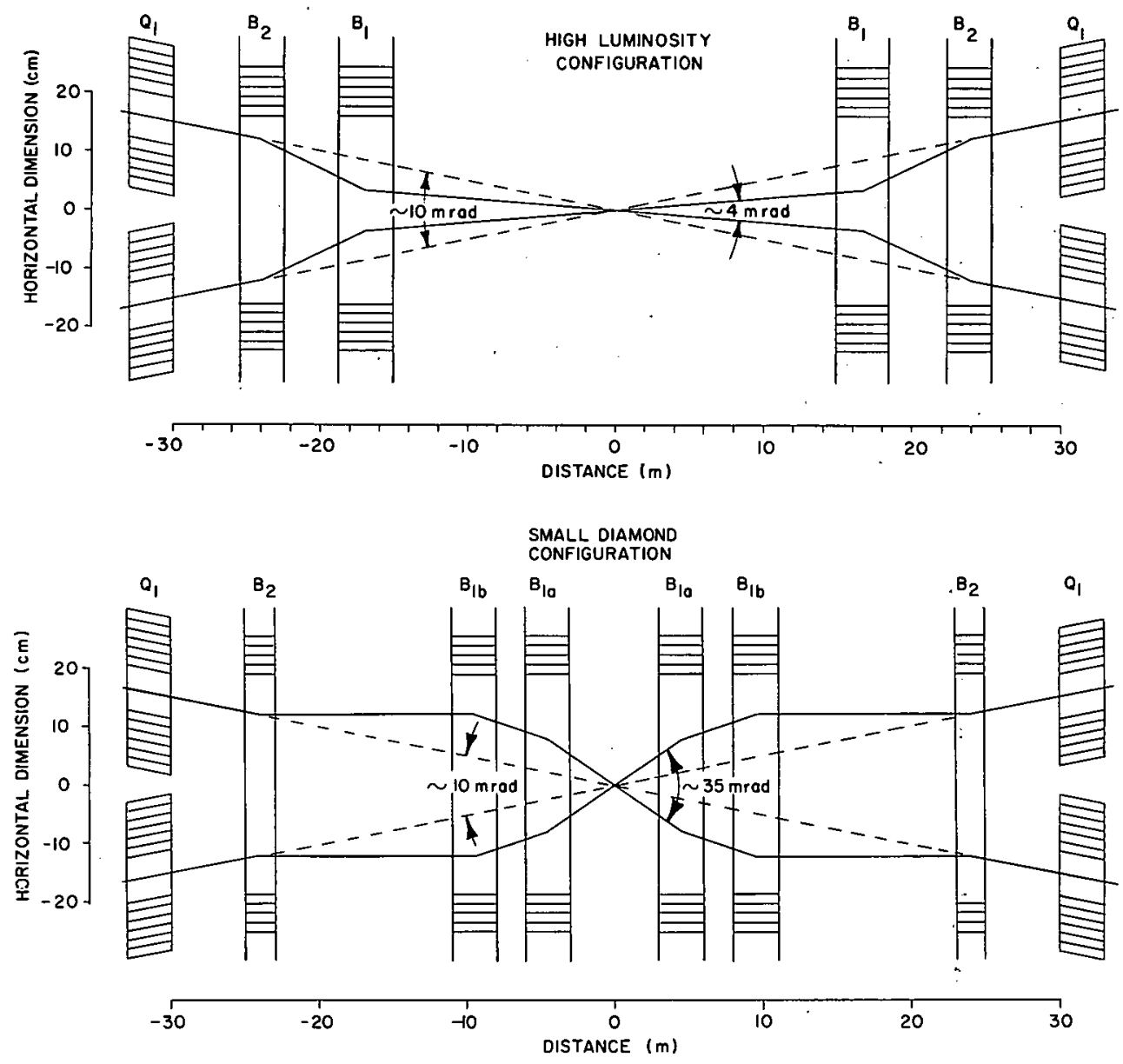

Fig. III.7. Beam trajectories for the high luminosity and small diamond configurations. 
fold symmetry, ISABELLE operation will shift to a reduced symmetry mode. This phase will be characterized by the introduction of nonstandard insertions through asymmetric quadrupole tuning and addition of appropriate insertion magnets. Although under such conditions the momentum aperture is reduced, there is also a corresponding reduction in the aperture required since a substantial fraction of the aperture required at injection is needed for the purposes of stacking and bunching for acceleration. In order to keep the beam-beam tune shift down during high- $\beta$ operation, the $t$ current will be reduced to about 2-4 A for these runs. Since experj ments requiring high- $\beta$ are generally those involving high counting rates, such low current conditions need be employed for only a small fraction of the total ISABELLE running time. 


\section{Introduction}

The design current of $8 \mathrm{~A}$ is built up by transferring the charge from the AGS repetitively into the storage rings. A beam stacking method similar to that used at the ISR has been adopted. It uses a reduced AGS intensity so that excessive phase space dilution can be avoided in the AGS. This makes possible the retention of high phase space density in the injection and stacking process and permits significant scraping of the beam during the stacking process. The latter is essential in obtaining clean beam stacks for colliding beam operation. Using synchronous bunched beam transfer, the beam is injected onto the injection orbit and slowly accelerated into the previously established debunched beam stack. On completion of the stacking process the beam is centered in the chamber and rebunched into three beam bunches for acceleration to the ultimate colliding beam energy. At the desired energy level the beam is allowed to debunch again and brought into collision with the beam in the second ring in the center of the interaction regions. Protective fast beam extraction systems have been incorporated in the design. They permit safe disposal of the energetic beams in external beam dumps. This will prevent serious component damage which would result if the beam, with its high stored energy and its high charge density, accidentally were to hit the vacuum envelope or the superconducting magnet environment.

\section{eam Transfer from the AGS}

The desired reduction of the AGS beam intensity is achieved by changing the multiturn injection into the AGS from the $200 \mathrm{MeV}$ linear accelerator to one-turn fast injection. Injecting in this manner and using a linac beam intensity of $114 \mathrm{~mA}$ will result in a $30 \mathrm{GeV}$ beam intensity in the AGS of $2.7 \times 10^{12}$ protons. At this energy the beam bunches will be shaped by reduction of the AGS 
acceleration voltage amplitude for better matching into the longitudinal acceptance of the storage ring injection rf system. Subsequent to this the beam will be ejected from the AGS by means of a fast bunched beam extraction system into the existing external proton beam transport channel in the AGS north area. The beam transfer lines to the ISABELLE rings will branch off from this beam line through an achromatic 18 degree bending section. Following this, beam transverse phase space definition is provided by 4 FODO cells incorporating a sequence of simple fixed collimator A 1 degree switching magnet provides for injection into either of two 60 degree achromatic bending sections plus straight dispersionfree beam matching sections channeling the beam either to insertion 5 or insertion 7 .

Since the beam transfer system need only be energized during beam stacking operations (nominally one hour in a 24-hour period) room temperature magnets are used. The overall geometry of the AGS-ISABELLE beam transfer system is shown in Fig. III.8 and the beam transfer parameters are given in Table 4.

Tab1e 4. AGS Parameters, as ISABELLE Injector

Energy $29.4 \mathrm{GeV}$

Intensity/pulse $2.7 \times 10^{12}$ protons

Momentum spread at transfer $(\Delta p / p) f_{w}$ Vertical normalized transverse phase space $1.0 \times 10^{-3}$

Horizontal normalized transverse phase space Longitudinal phase space $15 \pi \mu \mathrm{rad} \cdot \mathrm{m}$ $15 \pi \mu \mathrm{rad} \cdot \mathrm{m}$ $1.06 \mathrm{eVsec} / \mathrm{bunch}$

$a_{\text {Enclosing }} 95 \%$ of particles in phase space, $i . e .2 .5 \sigma$ value. Injection

In order to preserve higher lattice symmetry and benefit from existing straight sections, the standard experimental insertions wil1 be used for beam injection as shown in Fig. III.9. With a sequence of vertical bending magnets, Lambertson septa, and a current septum unit, the $30 \mathrm{GeV}$ beam is brought into near coincidence 


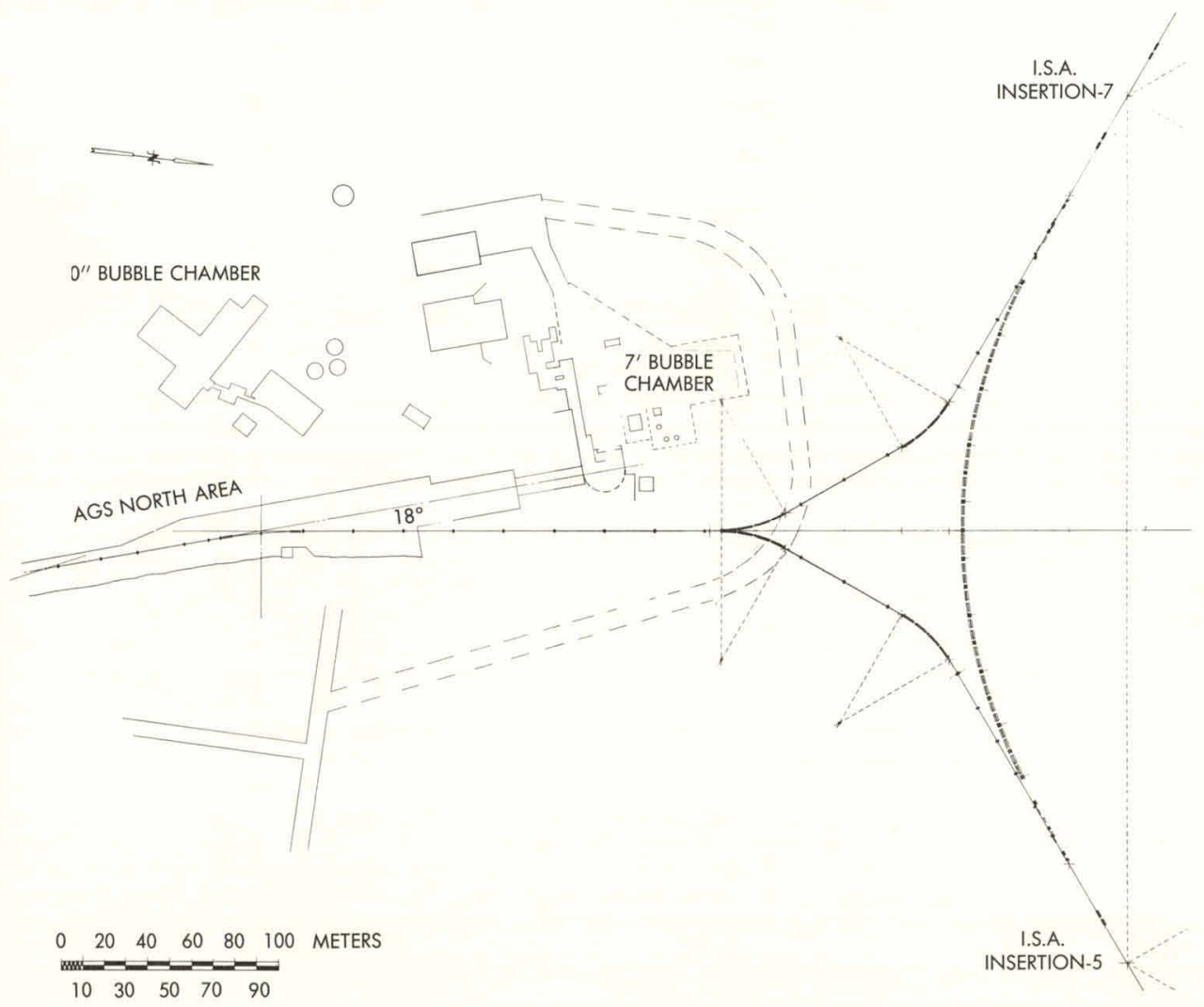

Fig. III.8. Sketch of the beam transport system between the AGS and the ISABELLE rings. 


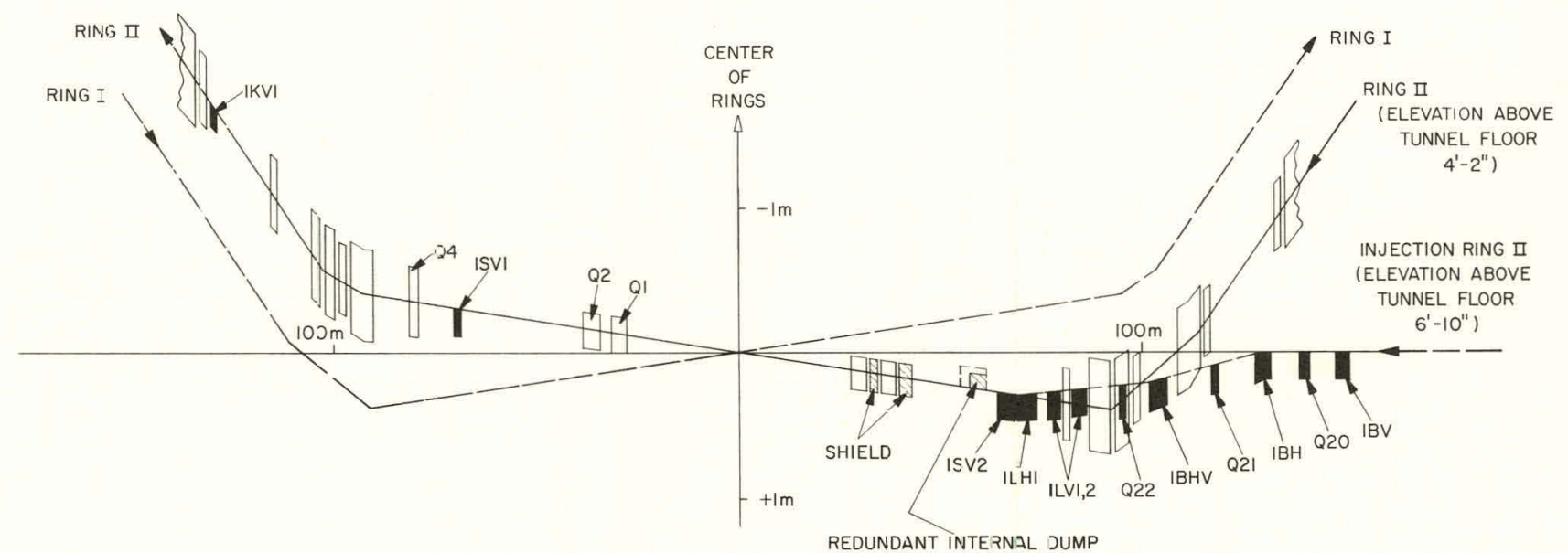

\begin{tabular}{|c|c|c|c|}
\hline DESIGN & ELEMENT & DEFLECTION & $\theta(\mathrm{mrad}), 30 \mathrm{GeV}$ \\
\hline KVI & KICKER & VERT. / PC & 1 \\
\hline ISVI & CURR. SEPTUM & VERT./PC & 1 \\
\hline Isv2 & CURR. SEPTUM & VERT./PC & 15 \\
\hline |LHI & IRON SEPTUM & HORIZ. I DC & 15 \\
\hline ILVI,2 & IRON SEPTUM & HORIZ. I DC & $28 /$ UNIT \\
\hline
\end{tabular}

Fig. III.9. Layout of the components in the " 7 o'clock insertion" of the rings used for beam injection. 
with the injection orbit in the free space between the quadrupole Q4 and the first (Q2/Q1) quadrupole doublet in the upstream end of the insertion. The injected beam is channeled through the insertion crossing domain with a maximum displacement of $<3 \mathrm{~cm}$. Downstream of the crossing point the beam passes through a trim septum unit located at the last insertion quadrupole and is directed to the vertical injection kicker which, in turn, will deposit the beam on the injection closed orbit. This kicker is located adjacent to the horizontally focusing quadrupole magnet of the first normal lattice cell where the momentum dispersion has a maximum value. Since the injected beam and low momentum part of the beam stack are separated in momentum by $1.0 \%$, the horizontal free space at this location between bunched injected beam and debunched (ful1) stack is $14.5 \mathrm{~mm}$. This is adequate for attenuation of the kicker stray field below a magnitude of $\left(\mathrm{B}_{\mathrm{B}} \mathrm{B}_{\mathrm{O}}\right) \leq 0.3 \%$. A split pole, fast vertical deflection dipole (van steenbergen 1975) may be used as injection kicker.

\section{Beam Stacking}

In the beam stacking process five groups of eleven AGS bunches wil1 be synchronously transferred into waiting matched buckets of the ISABELLE stacking rf system. This filling of 55 or the 57 buckets present on the injection orbit will take $4.8 \mathrm{sec}$ (at an AGS repetition period of $1.2 \mathrm{sec}$ ), after which acceleration toward the top of the existing stacked beam will begin. During the acceleration period the $\mathrm{rf}$ amplitude is gradually reduced such that before traversing the stack the buckets are tightly fitted around the beam bunches. Active feedback damping systems will be nployed to control any oscillation in the transverse or longiudinal phase planes.

The maximum voltage of the stacking rf system, $12 \mathrm{kV}$, will be used during the accumulation phase and early acceleration period which will be $\approx 0.13 \mathrm{sec}$ duration at a $\Gamma=\sin \varphi_{\mathrm{s}}=0.6$. This choice of $T$ represents an optimum between the conflicting 
requirements of a high stacking efficiency and a reasonable upper limit for the impedance $(\mathrm{Z} / \mathrm{n})$ at microwave frequencies presented to the beam by its surroundings. The voltage reduction will take $\approx 0.27 \mathrm{sec}$ while the final acceleration through the stack will take $\approx 4 \mathrm{sec}$ at a voltage that will vary from 1920 down to $1190 \mathrm{~V}$ as the top of the stack is reached. The total stacking time per cycle will be $\approx 9.3 \mathrm{sec}$. This will be repeated for a total of 62 cycles until $9.65 \mathrm{~A}$ are stacked of which $\approx 8 \mathrm{~A}$ will be within $1.00 \% \Delta \mathrm{p} / \mathrm{p}$.

The suggested mode of "stacking at the top" will result in a Low momentum "tail" to the stack, which will provide for greater stability against any self-bunching in the stack. The parameters of the stacking rf system are given in Table 5 and a preliminary design of the $4.45 \mathrm{MHz}$ rf cavity is given elsewhere (Giordano 1975). The associated low leve1 beam contro1 system, including provisions for phase lock and suppressed bucket operation will be similar to that employed at the CERN ISR (Hansen 1971). Table 5. Beam Stacking

Frequency $4.45 \mathrm{MHz}$

Frequency range $(\Delta f / f)$ $3.5 \times 10^{-5}$

Harmonic number

Maximum rf voltage $12 \mathrm{kV}$

Stacking time per cycle $9.3 \mathrm{sec}$

Number of stacking cycles

Stacking duration per ring (minimum) $\approx 10 \mathrm{~min}$ Stacked current with momentum tail Stacked current, after tail cleanıp Momentum width, final stack, $\Delta \mathrm{p} / \mathrm{p} \quad 1.00 \%$ $9.6 \mathrm{~A}\left(7.5 \times 10^{14}\right.$ protons $)$ $8 \mathrm{~A}\left(6.3 \times 10^{14}\right.$ protons $)$

Mounentum width, final stack, $\Delta \mathrm{p} / \mathrm{p}$

To prevent the development of instabilities during the stacking process and in the stack after it is formed, coupling impedances between the beam and the vacuum chamber and rf system must 
be kept 1ow. The most severe requirement exists for the single injected pulse while being accelerated into the stack. To prevent phase space dilution, the longitudinal coupling impedance $(\mathrm{Z} / \mathrm{n})$ must be kept below about $10 \Omega$ for all frequencies (Schnell 1975a). This will be achieved by avoidance of vacuum chamber envelope irregularities, the use of a shorting scheme for the gaps of the accelerating cavities and standard feedback techniques for the stacking rf system.

\section{Acceleration}

In order to accelerate the stacked beam, it will be rebunched by an $\mathrm{rf}$ system operating at the third harmonic of the revolution frequency $f=234.5 \mathrm{kHz}$. A peak $\mathrm{rf}$ voltage of $48 \mathrm{kV}$ per ring will be provided by four ferrite loaded cavities. These will be driven by low output impedance amplifiers. The conceptual arrangement of the rf low leve1, control and power drive system is shown in Fig. III.10. The amplifiers must be capable of providing large amounts of reactive power to compensate for beam loading. In order to prevent self bunching of the stacked beam prior to the bunching process and of the debunched beam at maximum energy, the impedance that they present to the beam should be $z / n \leq 12 \Omega$ per cavity. In addition to shorting the four accelerating gaps during the stacking process, they will again be shorted after the accelerating and deburuching cycle is completed.

Adiabatic rebunching of the beam can, in principle, be accomplished without phase-space dilution or loss of beam. However, scraping will be employed during the early part of the accelerating ycle to remove those particles present in the low energy tail -f the stacked beam as they will be outside the rf buckets. In this way the total phase-space area will be limited to $3760 \mathrm{eV} \cdot \mathrm{sec}$ which is equivalent to $\Delta \mathrm{p} / \mathrm{p}=1.00 \%$ stack width. During the early part of the acceleration cycle, both voltage and stable phase angle are programmed to provide the required bucket area without exceeding a bucket full height of $1.9 \%$ in momentum. Above $36.4 \mathrm{GeV}$ 


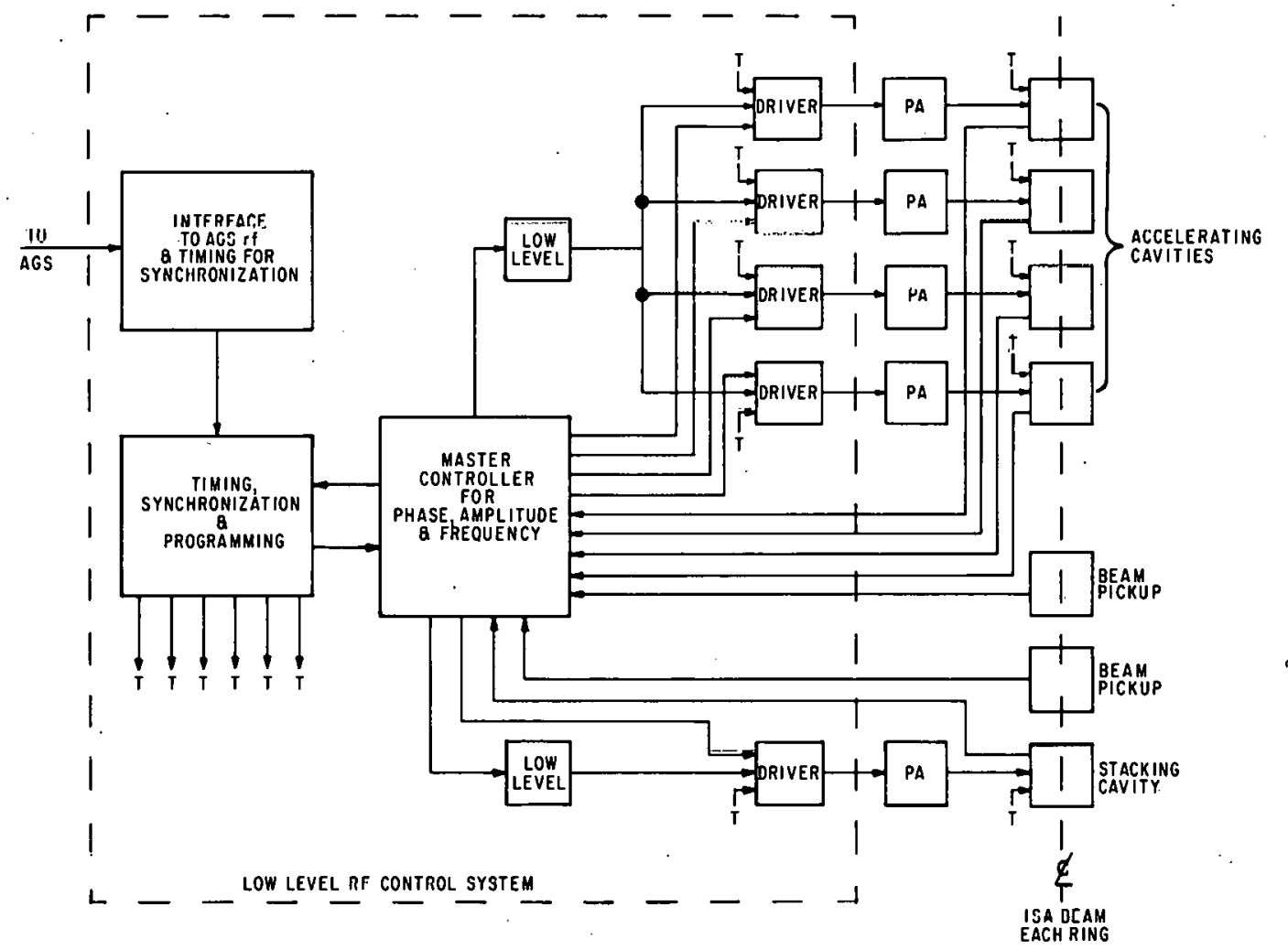

Fig. III.10. Block diagram for the low level rf control system. 
the maximum rf voltage of $48 \mathrm{kV}$ can be applied, and the stable phase angle will be programmed to ensure the required bucket area, which throughout the whole cycle will be $15 \%$ higher than the bunch area to avoid loss of particles. Voltages, stable phase angles and energy gain per turn will thus be:

$\begin{array}{lrrrr}\text { Energy } & 29.4 & 36.4 & 204 & 400 \\ \text { Voltage } & 22.6 & 48 \mathrm{kV} & 48 \mathrm{kV} & 48 \mathrm{kV} \\ \text { Stable phase angle } & 1.7^{\circ} & 13.1^{\circ} & 32.2^{\circ} & 32.2^{\circ} \\ \text { Energy gain per turn } & 0.68 \mathrm{keV} & 10.85 \mathrm{keV} & 25.6 \mathrm{keV} & 25.6 \mathrm{keV} \\ \dot{\text { B }} & 6.6 \mathrm{G} / \mathrm{sec} & 106 \mathrm{G} / \mathrm{sec} & 250 \mathrm{G} / \mathrm{sec} & 250 \mathrm{G} / \mathrm{sec}\end{array}$

The maximum field rise is $250 \mathrm{G} / \mathrm{sec}$ and the acceleration time is 4 minutes.

Although a second-harmonic rf system would provide greater security against longitudinal coupled bunch instabilities (Sacherer 1973), a third-harmonic rf system has been selected to permit collisions of bunched beams. The coupled bunch modes will be damped by Landau damping anyhow as the required coupling impedance for stacking is well below the rigid dipole mode threshold of $\mathrm{z} / \mathrm{n}=46 \Omega$ at $400 \mathrm{GeV}$, higher at lower energies. As the $\mathrm{z} / \mathrm{n}<$ $10 \Omega$ criterion for very large values of $\mathrm{n}$ is maintained during the acceleration cycle, the bunched beam will also be stable for those $\mathrm{n}$ modes where the wavelength is much shorter than the bunch (Hereward 1975).

During the rebunching process the chromaticity can be reduced by the ratio of the increase in momentum spread of the bunched eam to the stacked beam so that the size of the tune operating - egion will remain unchanged. This reduction from a $X \approx 2$, the value required for stability of the stacked beam against the transverse resistive wall instability, to $X \approx 1$ will still insure stability of the bunched beam against this instability up to mode $\mathrm{m} \approx 258$ (Sacherer 1974). For higher order modes the growth rates will be less than $12.5 \mathrm{sec}^{-1}$ (if they occur), which can be readily 
suppressed by a feedback damping system.

\section{Aperture}

Three modes of operation must be taken into account when considering the required aperture, i.e. injection and stack buildup at $30 \mathrm{GeV}$, bunched beam mode at $30 \mathrm{GeV}$, and debunched beam mode at $30 \mathrm{GeV}$ to $400 \mathrm{GeV}$ for physics utilization. For the latter mode a $7 \sigma$ value (Gaussian distribution) beam size is adopted. This criterion is based on ISR experience in order to keep background in the experimental detectors at tolerable levels (Resegotti 1976). Further, since it is essential that no significant fraction of the protons intercept the vacuum wall, which would result in driving the superconducting structure normal or in damaging the chamber envelope, it is also essential to specify a dump profile defined by the envelope of a hypothetical beam with transverse phase space several times that of the hard core $(2.5 \sigma)$ beam and momentum spread encompassing all operational momentum orbits. The magnitude of the dump profile changes azimuthally around the ring, however, it is so specified as to assure a minimum clearance (taken here as $3 \mathrm{~mm}$ ) from the physical vacuum chamber aperture. Protective fast beam extraction would be triggered if a preset fraction of the circulating current would exceed the dump profile, either due to changing closed orbit errors or beam growth associated with instabilities or resonance traversal. The tolerable proton flux outside the dump profile is assumed to be $\leqslant 0.1 \%$ of the maximum proton flux. This follows from the maximum caloric deposition density in the NbTi package of the superconducting magnets which leads to a permissible flux of $\leqslant 2 \times 10^{11}$ protons $/ \mathrm{cm}^{2}$ outside the dump profile (Petersen 1975).

The adopted aperture of $8.8 \mathrm{~cm}$ has been established by taking into account of factors such as the space required to stack the necessary current, the longitudinal impedance limit, the space required for closed orbit errors, and beam current limitations due to the pressure bump instability. The largest aperture requirement exists horizonta11y during beam injection. Details are shown in 
Fig. III.11, together with the cases for centered bunched beam and debunched stack for experimental operation. Because of the $7 \sigma$ clearance criterion during operation, a large vertical aperture requirement exists in the vertically focusing quadrupole adjacent to the beams crossing point. As a consequence, during. operation at energies below $50 \mathrm{GeV}$, the local $\hat{\beta}_{v}$ value would be limited to $\leq 220 \mathrm{~m}$ reducing the luminosity by a sma11 amount.

An allowance in aperture must be made for orbit sagitta, which for the present dipoles plus dewar ends, amounts to about $13 \mathrm{~mm}$ full value. However, taking advantage of the changing orbit functions along the length of the dipoles, the aperture allowance associated with sagitta can be reduced effectively to $5 \mathrm{~mm}$ full value, if the dipoles adjacent to the focusing quadrupole magnets are rotated by $1.6 \mathrm{mrad}$ from a symmetric inputoutput orientation.

In general, closed orbit errors introduced by magnet misalignments, field errors, etc. are correctable with the closed orbit correction system using dipole coils incorporated into the quadrupoles. The minimum value of horizontal and vertical orbit errors is determined by accuracy of position measurement and azimuthal distribution of correction dipoles. In addition to this, an operational. lower limit on the magnitude of the closed orbit errors is imposed by the long-term stability of measuring equipment. The magnitude of the residual central closed orbit errors is estimated to be about $1 \mathrm{~mm}$ rms horizontally when measured at the center of the regular focusing quadrupoles. owever, the magnitude of the errors varies across the width of the eam stack due to random errors in the location of the dipole current blocks, leading to closed orbit errors uncorrectable by a correction system using dipoles only. The projected random field errors (see Section III.5) together with an assumed effective $43 \%$ filling of the coil aperture lead to an uncorrectable minimum closed orbit error of $1.7 \mathrm{~mm} \mathrm{rms}$. A minimum allowance of $7 \mathrm{~mm}$ 


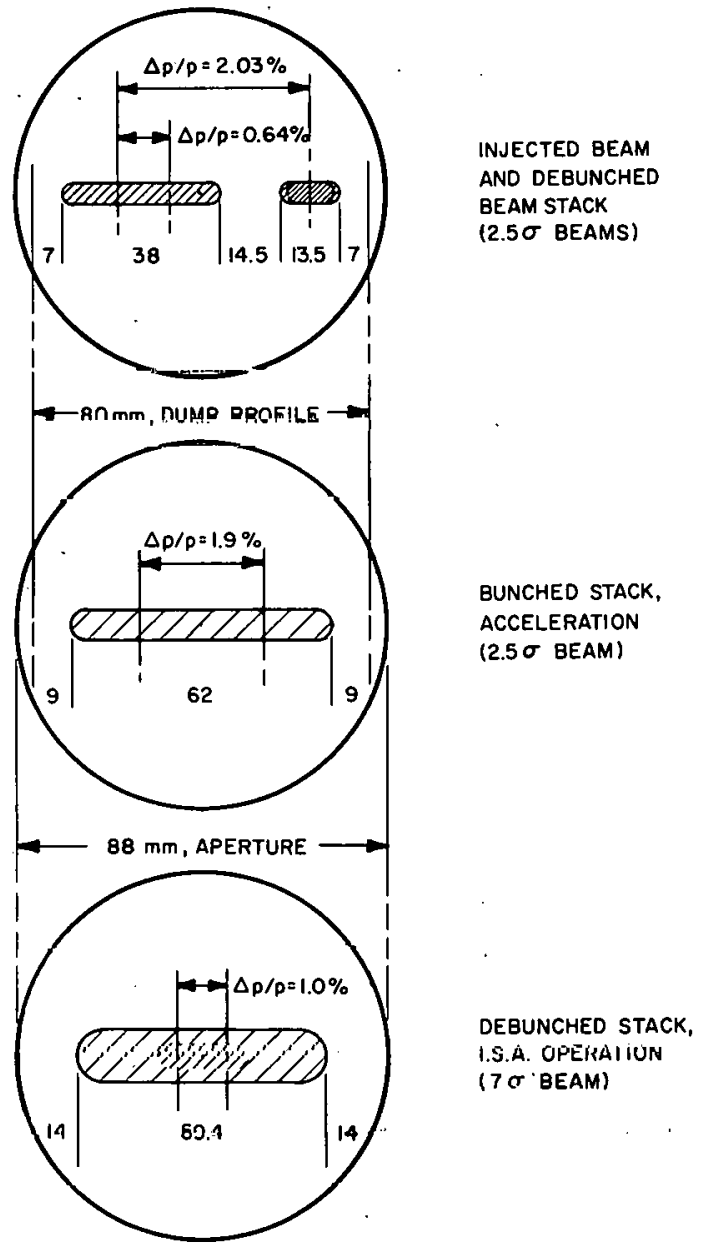

Fig. III.11. Aperture utilization at an azimuthal position corresponding to a maximum amplitude function. Bcam cnergy, $29.4 \mathrm{GeV}$. 
clearing space exists during the beam injection phase, which is most demanding in terms of aperture, to accommodate the total estimated closed orbit error.

\section{Protective Extraction}

Serious damage to vacuum chamber and adjacent magnets could result if they are hit by the high density energetic proton flux (stored energy $\sim 40 \mathrm{MJ}$ max.). Therefore, the capability for fast, single turn ( $12.6 \mathrm{\mu sec}$ ), near lossless ( $\leq 0.1 \%$ loss) beam extraction must be provided. Be defocusing the beam in the extraction channel outside the machine, it is possible to reduce the flux density to such a level that the beam may safely be deposited on a specially designed external absorber. Faults in the magnet, vacuum or safety systems, anomalous beam growth or closed orbit excursions that drive part or all of the beam outside a so called dump profile will trigger the fast extraction sequence. For the purposes of protective extraction the assumption must be made that the core of the beam may be located anywhere within the dump profile. Therefore, the first extraction septum must be located outside the dump profile boundary and the acceptance of the extraction channel must exceed that corresponding to the dump profile. The beam dumping reaction time is set by the rise time to full field of the first pulsed extraction septum units. A value of $50 \mu \mathrm{sec}$ has been adopted here, short compared with the fastest beam growth times observed at the ISR $(\simeq 1 \mathrm{msec})$. The external absorber will consist of a sequence of $\mathrm{Be}, \mathrm{C}, \mathrm{Al}$, and $\mathrm{Fe}$ materials. The static and dynamic stresses generated by the short duration, intense nuclear rascade in the dump material have been analyzed (Hauviller 1977), ndicating that a beam area enlargement factor of approximately 200 would be adequate to avoid damage in the external absorber. The general arrangement of extraction components, external trajectories and dump locations is shown in Fig. III.12. A redundant internal dump absorber will be added to provide for extra protection of the vacuum envelope and superconducting ring magnets. In case of failure of the primary system, the beam will impinge on an internal 


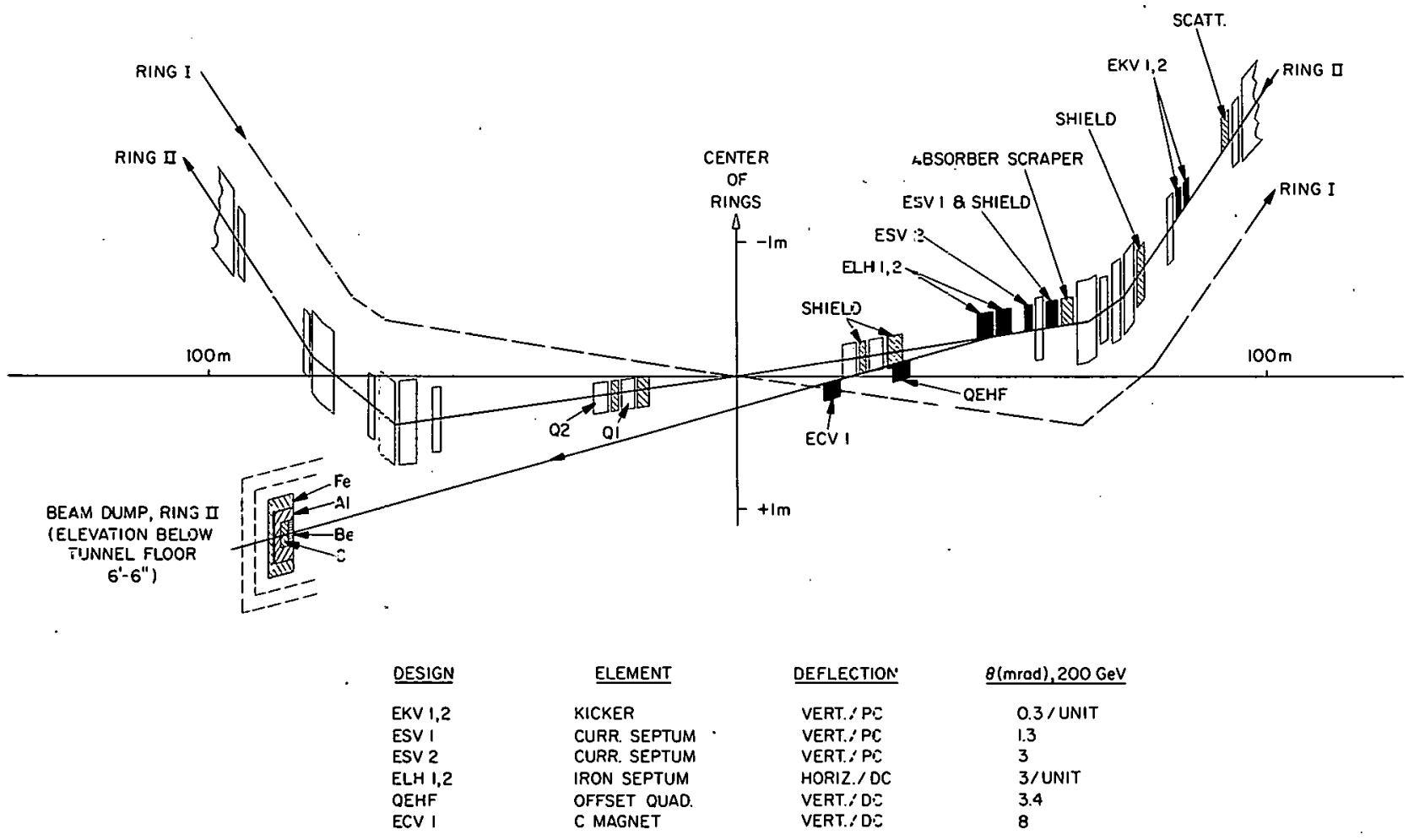

Fig. III.12. Layout of the components in the "5 J'clock insertion" used for beam ejection from Ring II. 
absorber where damage will be localized and readily repairable. This unit will also serve the beam scraper system, which will be used extensively during the stacking process to clean up unusable tails of the beam. Periodically, during operation for physics, it will be used to reduce experimental background. 
The ISABELLE magnet system will be superconducting. The lattice design, for a beam energy of $400 \mathrm{GeV}$, is based on $4.75 \mathrm{~m}$ long dipoles operating at $50 \mathrm{kG}$, and $1.69 \mathrm{~m}$ long quadrupoles with an operating gradient of approximately $6.1 \mathrm{kG} / \mathrm{cm}$. In addition, there are various special dipoles and quadrupoles required, some of which are of the conventional iron-copper type. The total number of each type of magnet in the two rings is as follows:

$\begin{array}{llr}\text { Cell Dipoles } & (4.75 \mathrm{~m}) & 720 \\ \text { Matching Cell Dlpoles } & (2.75 \mathrm{~m}) & 12 \\ \text { Ce11 F Quadrupoles } & (1.68 \mathrm{~m}) & 144 \\ \text { Ce11 D Quadrupoles } & (1.70 \mathrm{~m}) & 132 \\ \text { Insertion Quadrupoles } & (\mathrm{Q} 1, \mathrm{Q} 2, \mathrm{Q} 4) & 72 \\ \text { Low-B Insertion Quadrupoles } & (\mathrm{Q} 3) & 4 \\ \text { Skew Quadrupoles, Conventional } & & 48\end{array}$

Operation of the high-beta insertion will require 4 more Q3 quadrupoles. Correspondingly, operation of the high-luminosity or small-diamond insertions at some future time will require additional larger aperture dipoles as discussed in Section III. 2.

Figure III. 13 shows cross sections, and Fig. III.14 an isometric view of a main ring dipole magnet (McInturff 1977). The ideal cosine current distribution is approximated by six current blocks per quadrant containing $21,20,17,14,10$ and 5 turns respectively. The conductors are spread over their respective block areas by spacer turns braided from copper wire in order to minimize the peak field, which limits the magnet performance. In this configuration the two-dimensional peak field in the coil aperture is approximately $3 \%$ higher and the peak field at the ends $6 \%$ higher than the central field. Exact positioning of the blocks is determined in such a way as to suppress the six lowest undesired field harmonics. The ends of the coil blocks are 


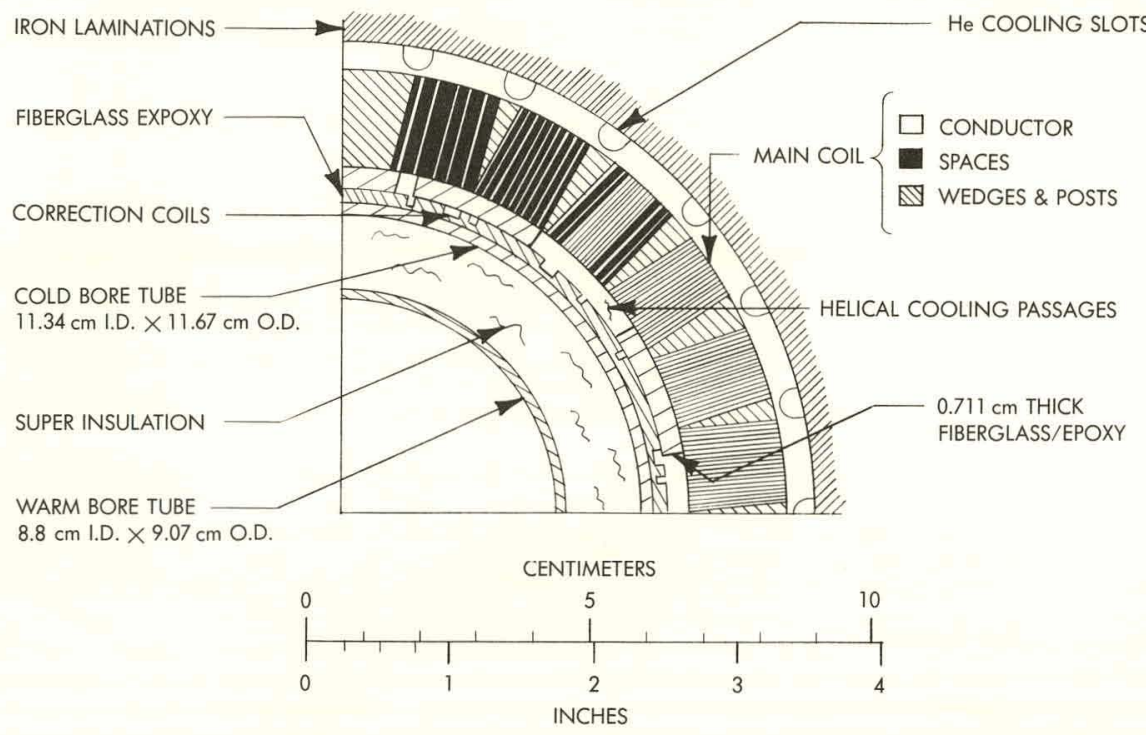

Fig. III.13a. Quadrant of dipole magnet, showing conductor arrangement, coil support structure, and bore tube arrangement.

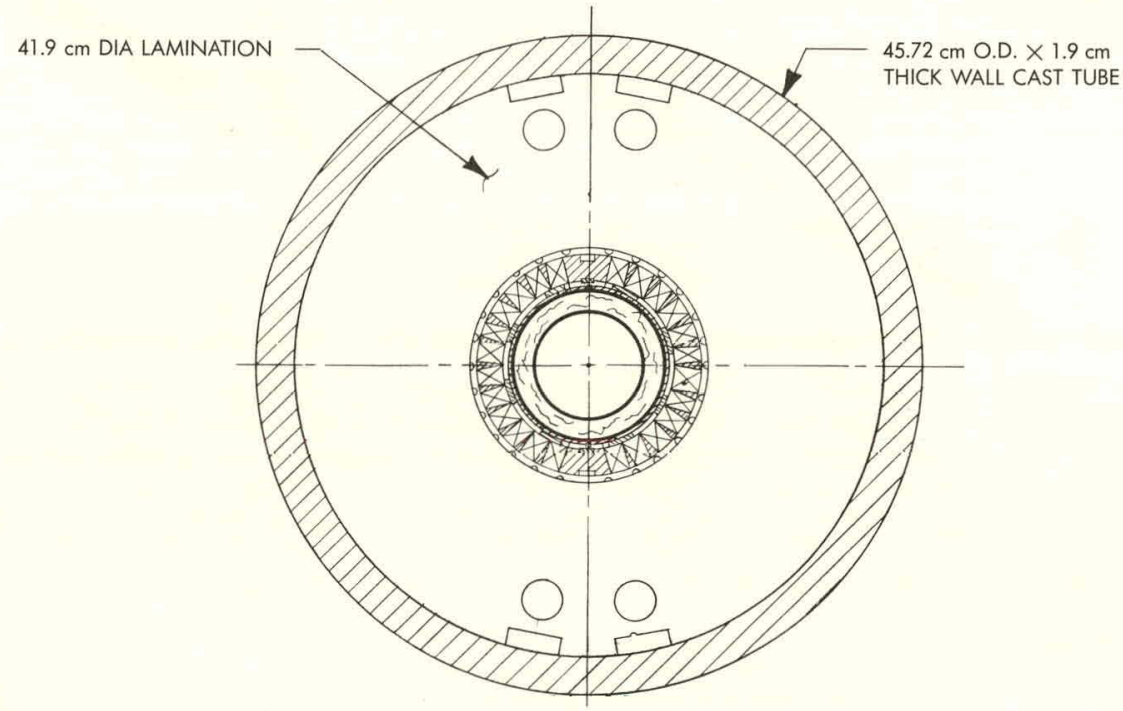

CENTIMETERS

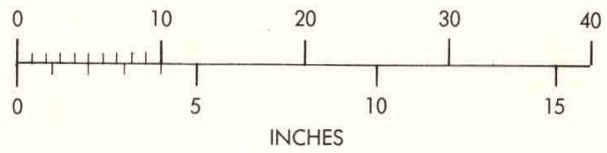

Fig. III.13b. Cross section of dipole magnet, showing iron shield and support tube. 


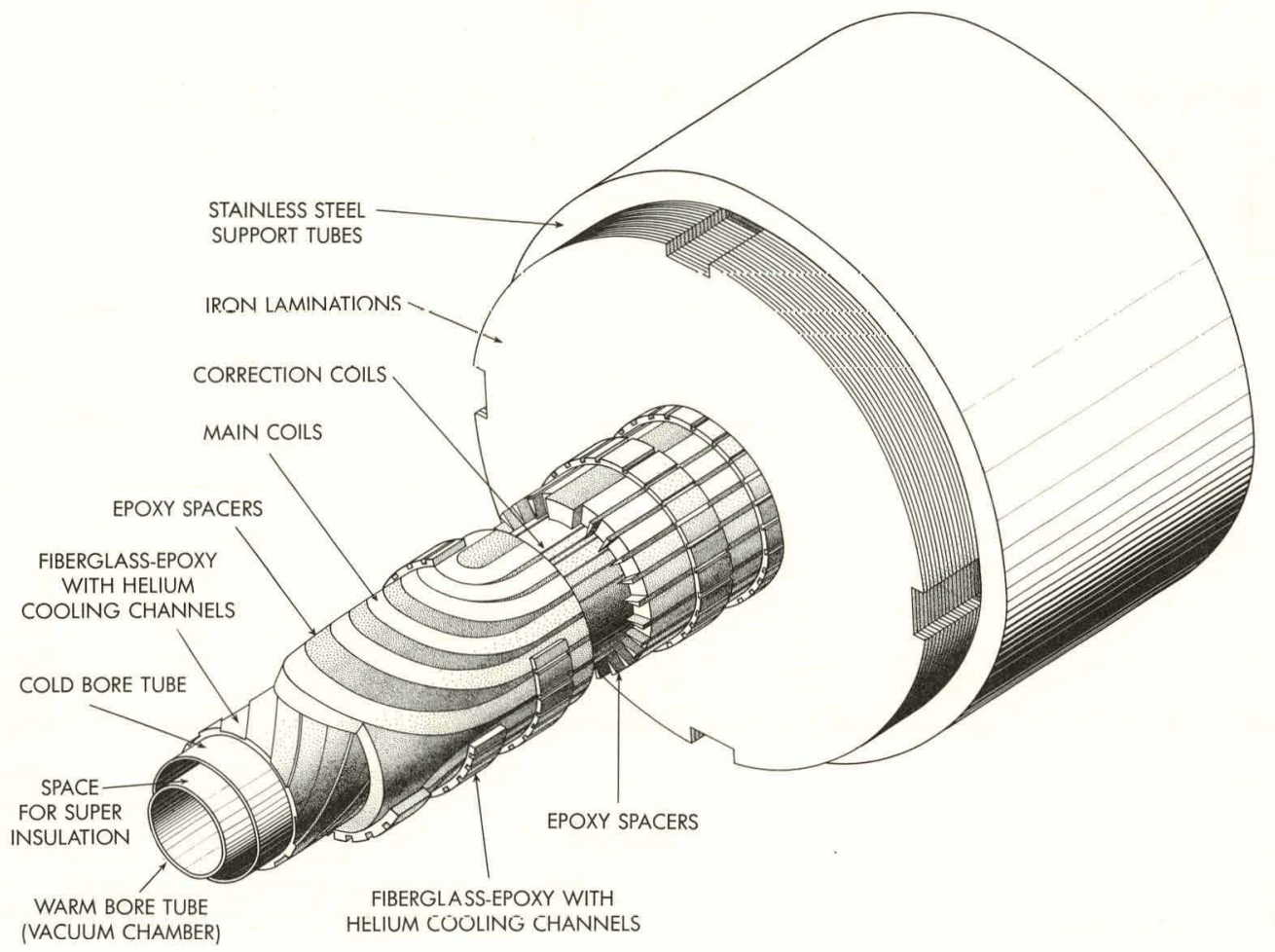

Fig. III.14. Isometric view of the dipole magnet, showing the configuration of the coil ends. 
displaced axially with respect to each other in such a way as to correct the total field integral seen by a beam passing through the coil ends.

The inner diameter of the coil is $13.09 \mathrm{~cm}$. The current blocks are wound with a single layer of wide flat conductor in the form of a braid (bare dimensions approximately $1.62 \mathrm{~cm} x$ $0.07 \mathrm{~cm})$. The braid is made up of 97 twisted composite wires $0.3 \mathrm{~mm}$ in diameter, each containing $\sim 500$ superconducting $\mathrm{NbTi}$ filaments of $10 \mu \mathrm{m}$ diameter. The $4.75 \mathrm{~m}$ magnets require $88 \mathrm{~kg}$ of superconducting wire each, or $1720 \mathrm{~m}$ of braid. The braid has a transposition length of approximately $25 \mathrm{~cm}$ and a packing density of $71 \%$. It is filled with an alloy of $\mathrm{Sn}-3 \mathrm{wt} \% \mathrm{Ag}$ to give mechanical rigidity and electrical stability. The wires in the braid have a $0.01 \mathrm{~mm}$ thick $\mathrm{Cu}-10 \mathrm{wt} \% \mathrm{Ni}$ jacket to increase the coupling resistance between wires and decrease eddy current effects.

The iron shield of the magnet, or core, provides mechanical constraint preventing the coil from moving due to the magnetic forces and as an integral part of construction, will also be at helium temperature. The shield also largely determines the dimensional accuracy of the coil with respect to longitudinal straightness and angular twist. The iron laminations are stamped in the form of unsplit annular disks of low carbon stee1, 1.25 mm thick. The cylindrical channels near the top and bottom of each 1amination, shown in Fig. III.13b, serve a dual function; a) they act as helium cooling channels, and b) control the sextupole harmonic field component as the iron saturates at high ield. The laminations are contained within an accurately achined heavy wall stainless steel core tube. The lamination length of the dipole is $4.75 \mathrm{~m}$, and its overall weight, including the iron core, approximately $5500 \mathrm{~kg}$. The small clearance between laminations and the core tube (maximum $0.2 \mathrm{~mm}$ ) necessitates that the stack of laminations be inserted after the tube diameter is increased by heating. Prior to inserting the coil, the bore of 
the laminations is honed. Coil insertion in the core is accomplished with the coil precooled in liquid nitrogen and the core at room temperature. This ensures an interference fit between core and coil of approximately $0.15 \mathrm{~mm}$ at operating temperature and, consequently, coil compression by the same amount.

End plates welded to the stainless steel core tube form a closed pressure vessel for containment of the helium coolant, thus eliminating the need for a separate inner helium vessel. Cooling is by high pressure gas, as outlined in Section III.6, circulating in channels on the outside and inside diameter of the coil (Figs. III.13a, III.14). The magnet coil and core assemb1y is mounted inside a concentric evacuated tank by supports having low heat conductivity. The annular space between the core support tube and vacuum tank wall contains an aluminum heat shield and multiple layers of superinsulation (Fig. III.15). The thermal insulation between the warm bore vacuum chamber and magnet coil must withstand baking to $300^{\circ} \mathrm{C}$ reached in outgassing the beam tube.

The main ring quadrupoles (Sampson 1977) are very similar to the dipoles in design. They utilize the same wide braid conductor arranged in a single coil layer approximating a cosine $2 \theta$ current density distribution, based on three current blocks per octant, containing 19, 15 and 8 turns respectively. The radial dimensions of the quadrupole cross section are basically the same as in the dipole, differing mainly in the arrangement of the field control windings as described below. The average quadrupole lamination length is $1.69 \mathrm{~m}$, and overa $\perp 1$ weight $2200 \mathrm{~kg}$. In addition to the regular ring quadrupoles there are a number of special quadrupoles required for the experimental insertions, as noted. They vary in length and excitation requirements depending on their function, but the design and fabrication technique is similar to that for the regular quadrupoles. However, they are different insofar as the iron core is not as thick. 


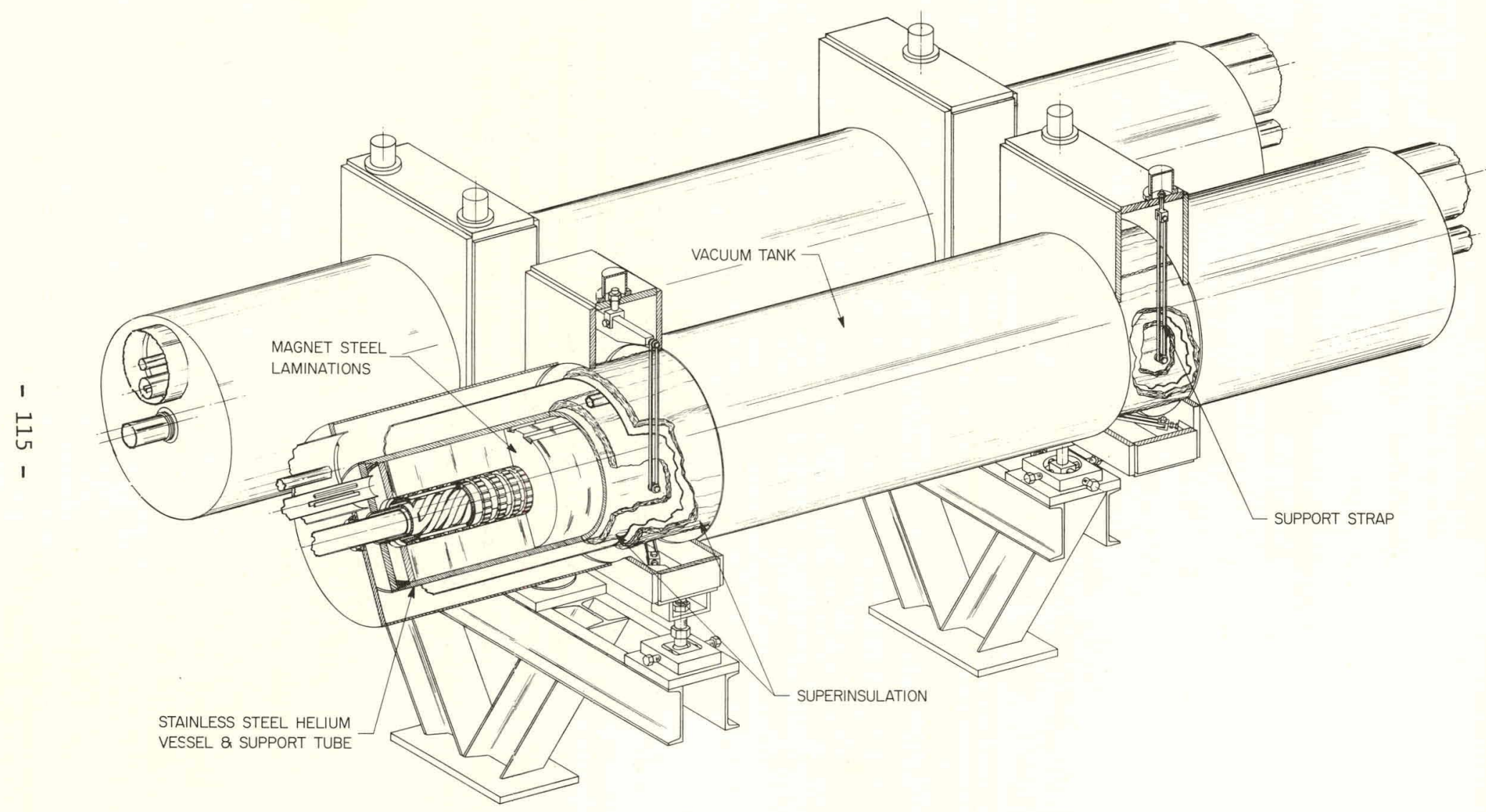

Fig. III.15. Isometric view of the dipole magnet assembled in the cryostat. 
Each magnet of the inner and outer ring will be contained in its own dewar, as noted above (Fig. III.15). This has the advantage that all vacuum joints are directly accessible for leak tests at room temperature and that the position of each magnet is separately adjustable. The arrangement of the three dipoles and a quadrupole in a half-cel1, the smallest unit of the regular ring lattice, is shown in Fig. III.16. Dipoles and quadrupoles are connected in series electrically and cryogenically. In order to minimize heat loads, the leads between all regular magnets of each sextant are kept cold. Hence, the magnet current can be chosen to meet other conditions. A value of $4.0 \mathrm{kA}$ at full excitation was selected so as to keep voltages induced during a quench within tolerable bounds.

The magnet quench protection system performs the following functions: 1) shunting the current around the quenching magnet, 2) venting the resultant warm gas so that it does not reach neighboring magnets, and 3) preventing the stored energy of the other magnets from being dissipated in the normal magnet. Located in the dewar are cold solid state diodes across each magnet and pressure relief valves to bleed off the warmed-up helium gases. External to the dewar is a resistive load and associated SCR device which are used to discharge a group of magnets, typically a half-sextant in the event of a quench of one unit. The function of this circuit is to extract and dissipate the stored energy of the remaining magnets in this group. 


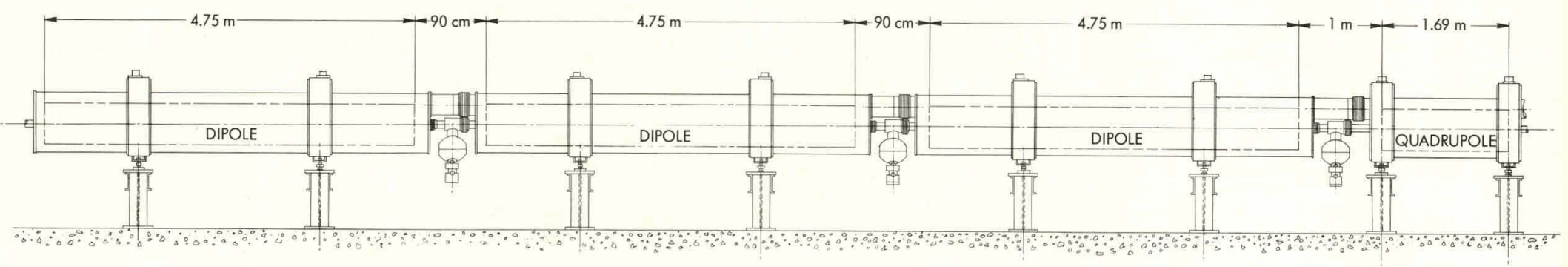

Fig. III.16. Half-cell magnet system, including three dipoles and one quadrupole. Between the dewars are the magnet interconnections and vacuum pumping stations. 
The dipoles and quadrupoles will each have sets of windings to provide complete control over the working line and to correct errors in the field shape of the magnets. These windings and the effects which will have to be corrected, are described in the following paragraphs.

Systematic errors in the magnetic field, which are the same in magnets intended to be identical, may be introduced by the conductor arrangement, by the construction of the coil ends, by iron saturation effects, by diamagnetic effects in the superconductor itself, and by rate-dependent induced currents (Parzen 1975a). The magnetic field errors are conveniently described by the field multipoles, $\mathrm{b}_{\mathrm{H}}$, defined by writing the median plane magnetic field error as $\Delta B_{v}=B_{0}\left(b_{0}+b_{1} x+b_{2} x^{2}+\ldots\right)$ and $\Delta B_{h}=$ $\mathrm{B}_{\mathrm{O}}\left(\mathrm{a}_{\mathrm{o}}+\mathrm{a}_{1} \mathrm{x}+\mathrm{a}_{2} \mathrm{x}^{2}+\ldots ..\right)$. Systematic effects introduce $b_{2}, b_{4}, b_{6} \ldots$ terms in the dipoles and $b_{5}, b_{9} \ldots$ terms in the quadrupoles. The tolerance on the sextupole term allowed after correction by correction windings is $b_{2} \leq 0.5 \times 10^{-6} / \mathrm{cm}^{2}$. The $b_{2}$ introduced by iron saturation effects at high fields is about $b_{2}=6 \times 10^{-4} / \mathrm{cm}^{2}$. Rate-dependent induced current effects introduce $a b_{2}$ which has been measured as $b_{2} \approx 10^{-5} / \mathrm{cm}^{2}$ at $\mathrm{B}=250 \mathrm{G} / \mathrm{sec}$. Diamagnetic ettects have been estimated to contribute $b_{2}=2.6 \times 10^{-5} / \mathrm{cm}^{2}$ at the low field end.

The dipoles will have $b_{2}$ and $b_{4}$ correction coils to correct the systematic field errors described above. The $b_{2}$ and $b_{4}$ coils will also be used to control the shape of the working line, in particular the chromaticity. For this reason there are two sets of $\mathrm{b}_{2}$ and $\mathrm{b}_{4}$ coils which are separately powered; one set is in dipoles near the focusing quadrupoles and the other set is in dipoles near the defocusing quadrupoles. Systematic field errors in the quadrupoles are corrected by $a_{5} b_{5}$ coil. In addition to the $\mathrm{b}_{5}$ coil, the quadrupoles will have $\mathrm{b}_{1}$ and $\mathrm{b}_{3}$ coils to control the 
shape of the working line. Altogether, there will then be 5 coils to control the shape of the working line; the $b_{1}, b_{3}, b_{5}$ coils in the quadrupoles and the $b_{2}, b_{4}$ coils in the dipoles. The $b_{1}, b_{3}$, and $b_{5}$ will also be separately powered in focusing and defocusing quadrupoles. Power supply tolerances on the excitation currents in all these correction coils will be such that the $\nu$-value across the beam will be controlled with an accuracy of about $\Delta \nu=0.001$.

Field errors that vary randomly from magnet to magnet are caused by random errors in the construction of the magnets (Parzen 1975b). The rms error expected in the position of the current blocks that make up the main coil is about $50 \mu \mathrm{m}$, which would result in rms random field error multipoles in the dipoles of $b_{0}=1.7 \times 10^{-4}, b_{1}=3.4 \times 10^{-5} \mathrm{~cm}^{-1}, b_{2}=6.6 \times 10^{-6} \mathrm{~cm}^{-2}$, etc. Another source of random field errors is the error in the position of the entire main coil relative to the iron shield. The rms error in this coil alignment is expected to also be $50 \mu \mathrm{m}$. This error introduces in the dipole magnets a random quadrupole term of $b_{1}=5.8 \times 10^{-5} \mathrm{~cm}^{-1}$. The overall tolerances established for the rms field error multipoles in the dipoles are thus $b_{0}=1.7 \times 10^{-4}, b_{1}=6.8 \times 10^{-5} \mathrm{~cm}^{-1}, b_{2}=6.6 \times 10^{-6} \mathrm{~cm}^{-2}$, etc. and correspondingly for the quadrupoles $\mathrm{b}_{1}=4.4 \times 10^{-5} \mathrm{~cm}^{-1}$, $b_{2}=1.0 \times 10^{-5}=m^{-2}$, etc. The random field errors produced by errors in the construction of the magnets have been measured on model magnets (McInturff 1977). The random field errors thus measured are consistent with the theoretical results for the -ssumed error in the position of current blocks of $50 \mu \mathrm{m}$.

The random dipole field errors, $b_{0}$ and $a_{0}$, whose primary effect is to displace the central orbit, will be corrected by the central orbit correction system (Parzen 1975a, Edwards 1975b). The largest source of random dipole field errors is random errors in the position of the quadrupoles, which is about 
$0.25 \mathrm{~mm} \mathrm{rms}$. Other sources include random errors in the rotational alignment of the dipoles, and errors in the construction of the magnets. The central orbit will be corrected by movement of the quadrupoles and by a system of dipole correction coils located in the quadrupoles. Vertical and horizontal dipole correction windings will be put in the focusing and defocusing quadrupoles, respectively, and will be capable of being powered separately. The dipole correction coils near the beam crossing points will also be used for local steering of the beams. The central orbit correction system will reduce the central orbit displacement to about $1 \mathrm{~mm}$ around the ring, and the vertical position of the beam at the intersection points will be controlled within less than $0.1 \mathrm{~mm}$.

Another effect of the random errors is to generate imperfection resonances. Calculations of the widths of the resonances (Gareyte 1975) and experience at the ISR indicate that one can avoid the harmful effects of these resonances by careful control of the working line shape. No correction coils are provided for most of the possible resonances, but there is space to add such correction elements after the accelerator is operating, if this proves desirable. Skew quadrupoles will be provided to control two imperfection effects which might be harmful (Gareyte 1975, Chasman 1976), which are coupling of the vertical and horizontal betatron oscillations and vertical dispersion of the central orbit at the crossing point.

Table 6 lists all the correction coils, the location of the correction coil, the maximum correction provided, and the required accuracy of the correction. 
Table 6. Correction Coil Table for $400 \mathrm{GeV}$ ISABELLE

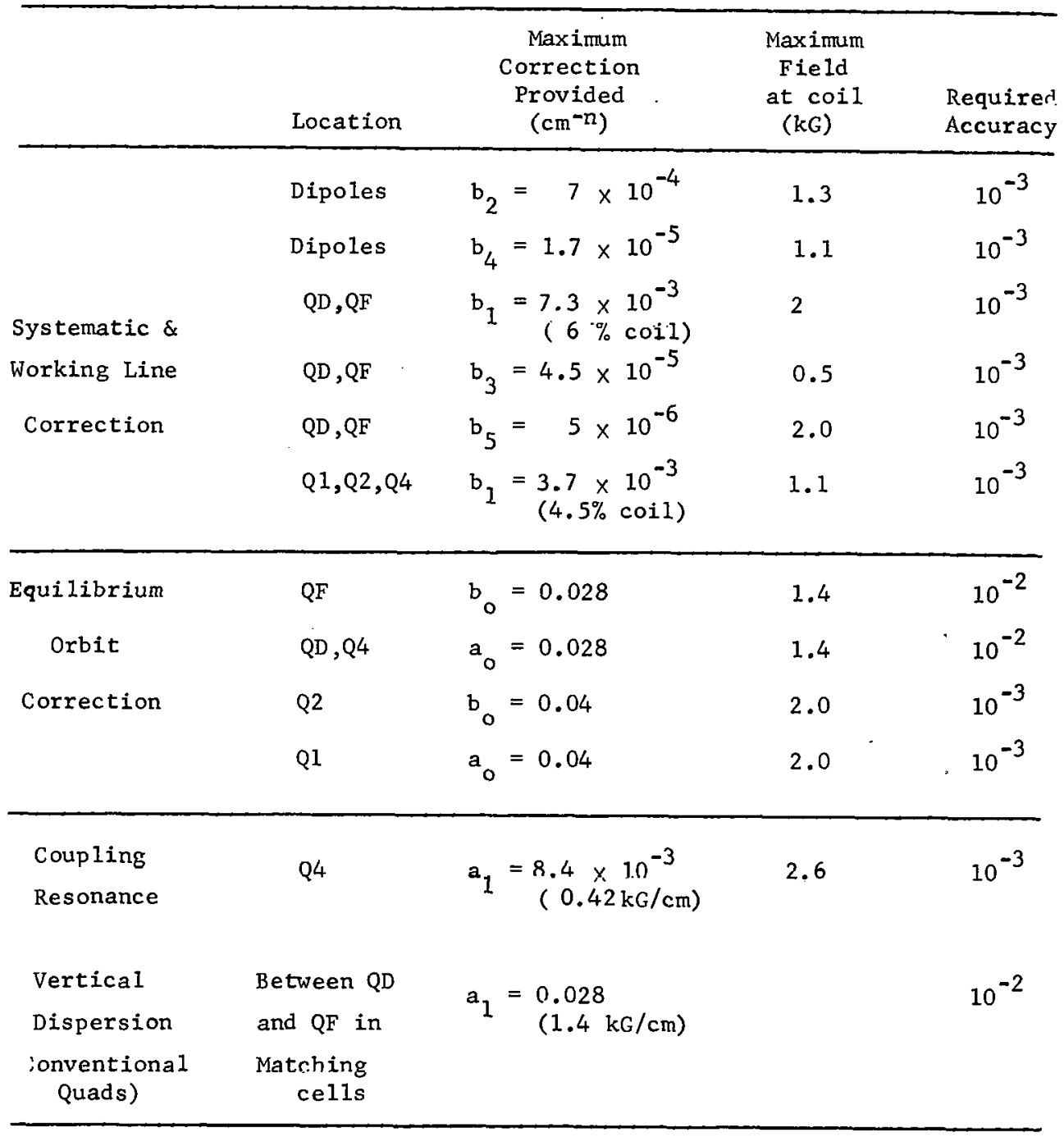


The insertion quadrupoles Q1, Q2 and Q4 are powered in series with the main dipoles, with the exception that Q4 is separately powered in the low- $\beta$ insertion. Because of this, these quadrupoles need to have quadrupole trim coils to compensate for the difference in the saturation effects in the insertion quadrupole and in the main dipoles and also to alter the Q1 and Q2 quadrupoles for the low- $\beta$ insertion. 
The nominal operating temperature for the ISABELLE superconducting magnets is $3.8 \mathrm{~K}$. This temperature can be attained only by the use of a refrigerator which uses helium as the working fluid. We have analyzed various systems for the production and distribution of refrigerant for the ISABELLE magnet system. The most cost effective system utilizes helium at supercritical pressure to cool the magnets and this system has been incorporated in the magnet and refrigerator design (Brown 1976).

The estimated steady-state heat load at $3.8 \mathrm{~K}$ for ISABELLE is $13 \mathrm{~kW}$ (see Table 7). The refrigerator is designed to produce $19 \mathrm{~kW}$, at this temperature. The refrigeration capacity is adequate to provide cooling for the ISABELLE magnets and to provide refrigeration for superconducting magnets used by experimenters. Refrigeration is also required at a mean temperature of $55 \mathrm{~K}$ to cool the heat shield. The estimated load at this temperature level is $17.5 \mathrm{~kW}$ and refrigeration capacity of $35 \mathrm{~kW}$ is provided. A simplified schematic of the distribution system is shown in Fig. III.17. The helium which cools the magnets leaves the refrigerator at a pressure of 5 atm and a temperature of $2.6 \mathrm{~K}$. As many as 46 magnets (one-half sextant of one ring) will be cooled in series, and the steady-state design temperature for the last magnet is $3.8 \mathrm{~K}$. This temperature rill rise to no higher than $4.2 \mathrm{~K}$ during the acceleration cycle, ind then return to $3.8 \mathrm{~K}$ or below during storage ring operation (Shutt 1976). Some flow will be removed from the refrigerant stream as required to cool magnet power leads, and to provide refrigeration for experimenters detector magnets. This flow returns to compressor suction at $300 \mathrm{~K}$. 


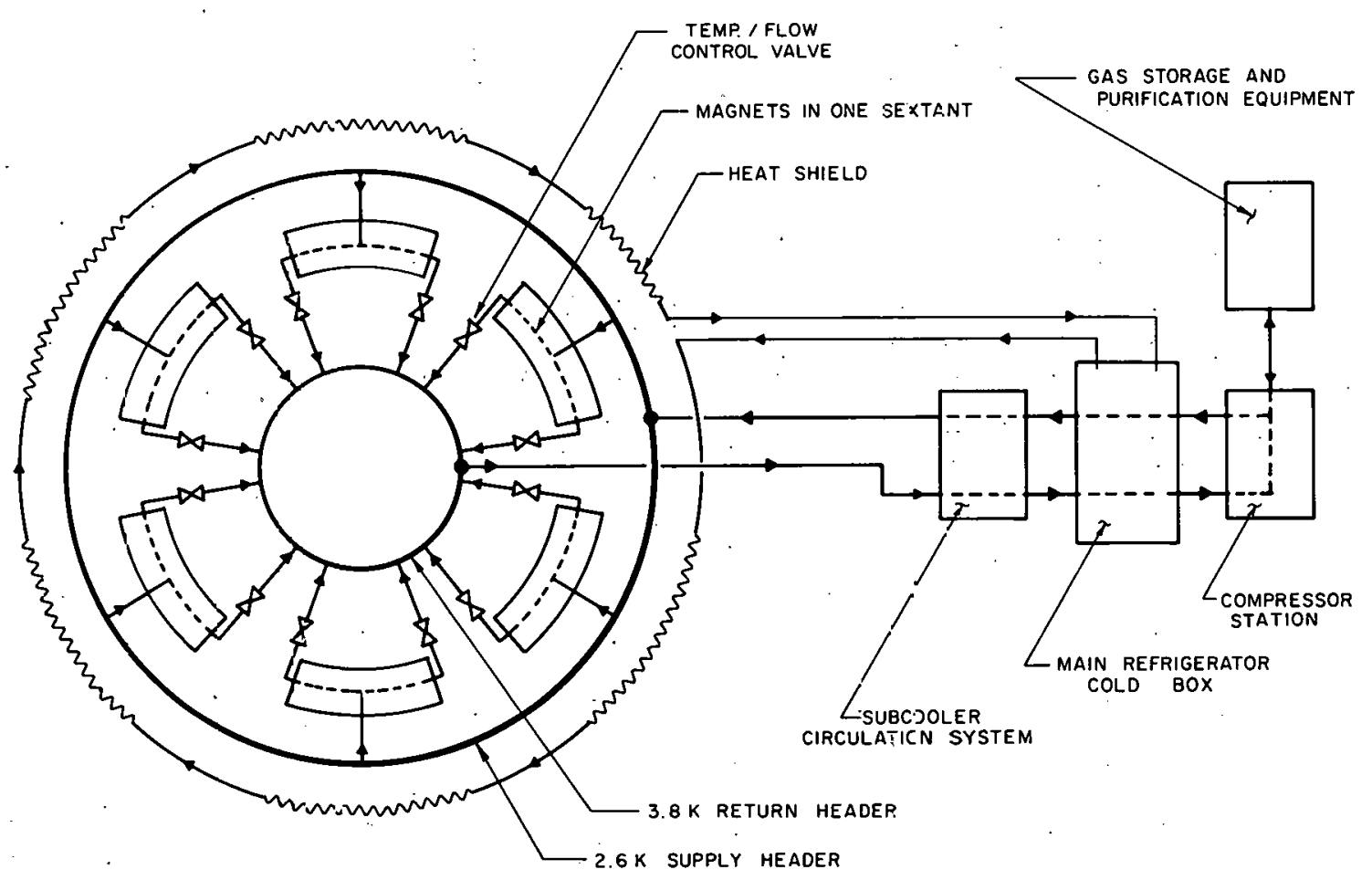

Fig. III.i7. Block diag=am showing the refrigerator distributior system for the ISABELLE rings. 
Magnets

Support Losses

Radiation

Connecting Piping

Instrumentation

Magnet Power Leads

(Total equivalent refrig. load)

Main Current

Insertion Quads

Correction Coils

Closed Orbit Coils

Distribution System

Piping

Valves

Pump Losses

Experimental Area Detectors

(1500 1iters/hr. equivalent)

Total Estimated Heat Load

Refrigerator Design Capacity

100
2700
1100
500

$55 \mathrm{~K}$

5200

4600

1100

500

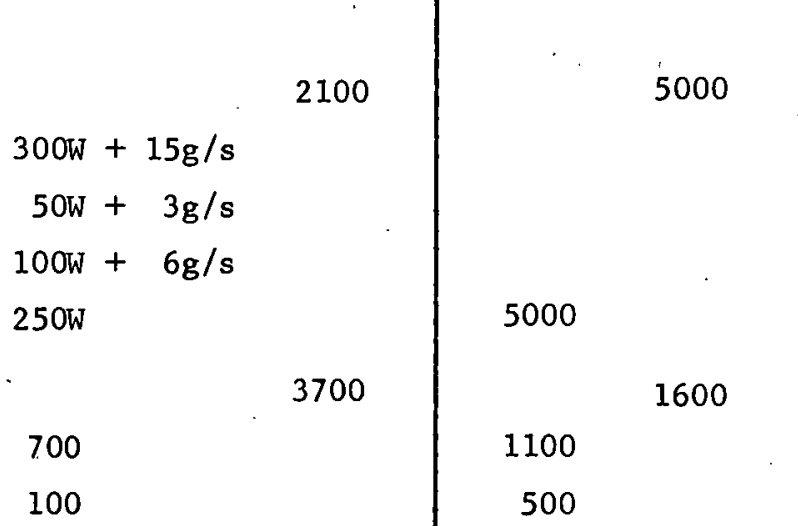

2800 
A single refrigerator is proposed for ISABELLE. Sufficient distances can be spanned without undue pressure drop or other penalties using the distribution system envisaged so that all the refrigeration can be supplied from a single point. More than one smaller refrigerator could have been used at this point, but a single unit was chosen primarily on the basis of reliability and cost considerations.

The refrigerator design utilizes the $\mathrm{Claude}$ cycle without liguid nitrogen precooling. Cooldown of the entire magnet system requires about 15 days. Input. power required for the refrigeration compréssörìs is $16 \mathrm{MN}$. 'lhe compressors will be dry screw type. Four stages of compression are required for the pressure ratio used in this cycle.

The radiation heat shield and support heat intercept cooling for the ISABELLE magnets will be provided by a gas stream which leaves the cold box at a temperature of $42 \mathrm{~K}$ and returns at $68 \mathrm{~K}$. The inlet pressure to the shield header is $15 \mathrm{~atm}$ and the pressure drop through the system is estimated at $3 \mathrm{~atm}$.

The final element of the refrigeration system is the subcooler which is shown schematically in Fig. III.18. The load, as seen by the refrigerator, is comprised of the refrigeration load below $3.8 \mathrm{~K}$ (Point No. 1), a liquefaction load (Point Nos. 2 and 3 ) and the heat of compression from the circulating compressor (Point No. 4). Mass removed from the circulating stream at Point Nos. 2 and 3 is replaced by the makeup supply at Point No. 5 . A large mass flow of helium is available for distribution to the magnets and is a separate loop from the refrigerator flow except fi the make-up at Point No. 5. This circulating stream is cooled in the subcooler (Point No. 6) to a temperature of $2.6 \mathrm{~K}$ at Point No. 7 and then distributed through the supply header to the magnets.

In order to attain the low temperature desired in this system, 


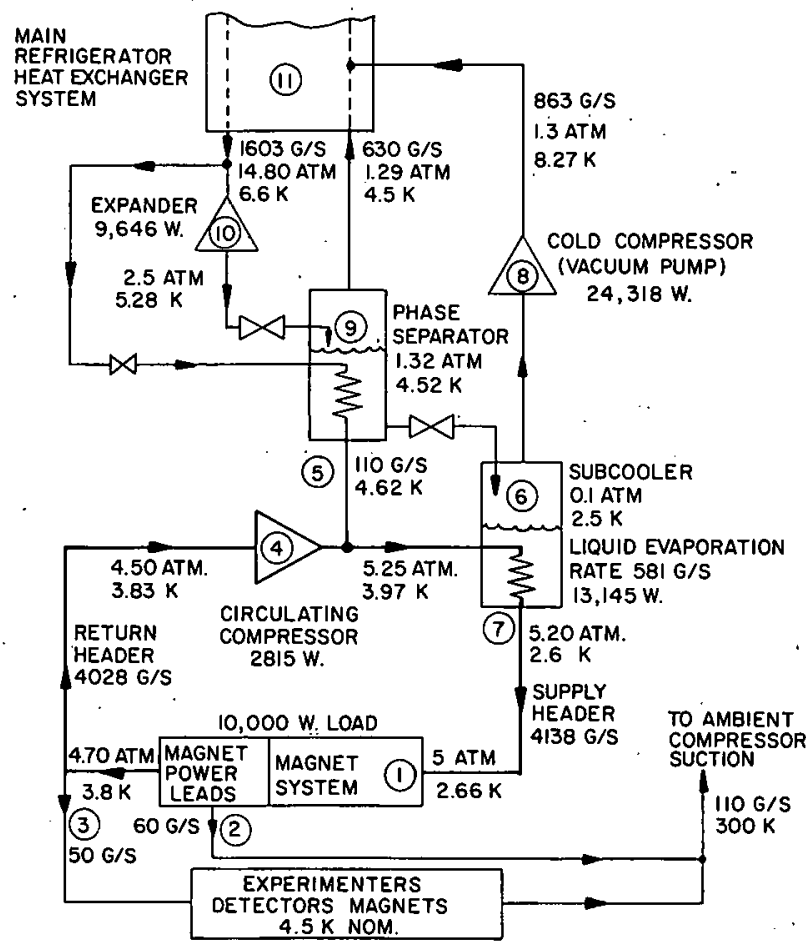

Fig. III.18. Functional schematic for the subcooler. 
a bath of liquid helium at subatmospheric pressure is required in the refrigerator. The subcooler (Point No. 6) operates at $0.1 \mathrm{~atm}, 2.5 \mathrm{~K}$. The pressure is maintained at the required low value by pumping the vapor from the boiling liquid with a turbocompressor (Point No. 8). $13.1 \mathrm{~kW}$ of refrigeration is produced at a temperature level of $2.5 \mathrm{~K}$. This is equivalent to the $19.0 \mathrm{~kW}$ required by the load at a higher temperature level. The make-up supply is precooled in the phase separator (Point No. 9) before being introduced to the circulating stream. Liquid from the phase separator undergoes a Joule-Thompson expansion as it flows to the subcooler. A turboexpander (Point No. 10) extracts work from the main refrigerator flow stream and produces refrigeration at a low temperature level. The low pressure gases return to the main refrigerator (Point No. 11) as shown in the schematic.

No liquid helium storage facilities are required for this system. The helium gas required to fill and operate the system will be stored in tanks near the refrigeration building. In the event of refrigerator failure, electric power failure or other system faults, no helium will be lost because the helium in the magnets is at high pressure and can be vented directly to the scorage tanks. 'l'heretore, no recovery compressor is required. Low pressure buffer or surge tanks are provided on the suction side of the compressors.

The piping required to distribute the refrigerant is vacuum insulated. Its design uses a single vacuum jacket which contains both the supply, return and heat shield piping. A heat shield surrounds the supply and return piping and reduces its heat gain to near zero.

Because so many magnets are cooled in series, only a relatively few control elements are required. The temperature of the refrigerant as it exits from each series of magnets will be monitored 
and will serve as the control point for flow control of each of the parallel cooling circuits. An overall system control circuit will optimize the production and distribution parameters to attain the most efficient operation under any operational load conditions which occur. 
The density of residual gas molecules in proton storage rings must be lower, by several orders of magnitude, than that in conventional accelerators. This is necessary in order to reduce beam loss and the accompanying radiation background in the experimental insertions caused by nuclear scattering. The vacuum system design, therefore, calls for a hydrogen pressure of less than $3 \times 10^{-11}$ Torr in the lattice sections of the rings and, with additional pumping, lower than this in the experimental insertions. At these pressures the beam los from gas scattering is smaller than 1 ppm per min and the total background in the experimental straight sections is estimated to be about $7 \times 10^{5}$ particles per second for a circulating current of $8 \mathrm{~A}$. Beam growth due to multiple Coulomb scattering is calculated to be negligible at the operating pressures. At $10^{-11}$ Torr it is still necessary to prevent the beam from becoming neutralized, that is, to prevent the trapping of the electrons and negative ions produced by the beam-gas collisions. This beam clearing will be achieved in ISABELLE by a system of clearing electrodes placed at regular intervals around the machine. Presently a distribution of 1512 clearing electrodes with electrical potentials of about $\pm 5 \mathrm{kV}$ is envisioned. The average ratio of trapped electrons to circulating protons is calculated to be 1 to $2 \times 10^{-4}$ at full beam current (Herrera 1976 a). For this degreee of neutralization the changes in the betatron tunes of an unbunched beam can be determined. Thus, at injection energy one obtains (Chao 1975) a net space-charge vertical tune depression of $14 \times 10^{-3}$, while at $400 \mathrm{GeV}$ one obtains a value of $2 \times 10^{-3}$. The corresponding horizontal tune changes are respectively $11 \times 10^{-3}$ and $2 \times 10^{-3}$. The accompanying tune variation across the beam, from center to edge, is about $10 \%$ of the central depression. These net v-value 
changes are small and can be compensated for by proper adjustment of the tune and chromaticity corrections of the lattice (Smith 1975).

The presence of trapped electrons in the beam can also give rise to a transverse coherent motion of the protons, coupled to the electrons oscillating in the potential well of the beam. This "e-p instability" has been observed at the CERN ISR (Hereward 1971, Schne11 1975 b), and at the LBL Bevatxon (Grunder 1971). Though difficult to calculate, the degree of neutralization at which this instability can start is very much dependent on the spread in frequencies of the oscillating electrons (Keil 1971). The ISABELLE machine has characteristically large variations in beam size as a function of azimuthal position. Since this results in a large spread of electron frequencies, the onset of the e-p instability is inhibited at a neutralization below about $10^{-4}$.

Experience with the CERN ISR has shown that the most serious limitation on current is set by the beam induced pressure rise, known as the "pressure bump phenomenon" (Fischer 1972). Qualitatively, this effect is caused by ionized residual gas molecules which are propelled by the electric field of the beam, strike the vacuum chamber wall, and thereby liberate adsorbed molecules in sufficient quantity to increase the gas pressure. At a certain value of current, I crit, this pressure rise leads, avalanche-like, to a local pressure peak and the destruction of the beam. For the geometry of the ISABELLE vacuum system $(8.8 \mathrm{~cm}$ i.d. circular vacuum chamber, $5 \mathrm{~m}$ distance between pumps, each with a pumping speed of sout $1000 \mathrm{1} / \mathrm{sec}$ ), the theory (Halama 1975) predicts a product 'I $I_{\text {crit }} \approx 40 \mathrm{~A}$. The desorption coefficient $\eta$ (defined as the net number of molecules desorbed per incident ion), characteristic of the chamber wall, depends upon the surface preparation and bake-out temperature used for the chamber material, as well as upon the mass and energy of the bombarding ions. In ISABELLE, 
though the residual gas will be mainly hydrogen, the onset of the pressure bump will manifest itself by a rise in the pressure of CO. It is then expected that, for a beam to wall potential of $2 \mathrm{kV}$, the desorption coefficient will be less than 3 , and, therefore, that the beam current limit will be greater than $10 \mathrm{~A}$. Such an upper bound on the value of 7 is consistent with the extensive experience existing at the CERN ISR where the chamber is made of stainless steel and a bake-out to $300^{\circ} \mathrm{C}$ is used. Experiments which are in progress at BNL have yielded 7 values close to zero for a $200^{\circ} \mathrm{C}$ bakeout. It should be mentioned here that the use of a vacuum chamber operating at liquid helium temperatures has been considered (Grand 1972) but abandoned in view of the physical and engineering uncertainties associated with such cold systems (Halama 1975).

Preliminary experiments on the CERN ISR (Gröbner 1977) have cast some doubt as to whether the use of a warm aluminum chamber, previously considered for ISABELLE, is consistent with a high intensity proton beam in a storage ring. The present ISABELLE design which calls for a stainless steel chamber (vacuum fired at $950^{\circ} \mathrm{C}$ and jacketed with copper for bake-out purposes) operating at room temperature and bakeable to $300^{\circ} \mathrm{C}$, therefore, represents a conservative approach. 'l'o insulate the warm bore tube trom the magnets at $4 \mathrm{~K}$, it is planned to use 35 layers of aluminized Kapton. The heat load is then about $0.45 \mathrm{~W} / \mathrm{m}$. With regard to the resistive wall instability, a stainless wall will give rise to a larger resistive component of impedance than an aluminum wall. However, when this resistive part is combined with the reactive part, the net result is that a betatron tune spread of about 0.02 is still sufficient to damp the instability.

The pumping is provided by conventional Ti-sublimation and sputter-ion pumps for nongetterable gases. Recent measurements (Halama 1977) have shown that a 30 liter/sec ion pump combined with a titanium getter is sufficient to lower the pressure below 


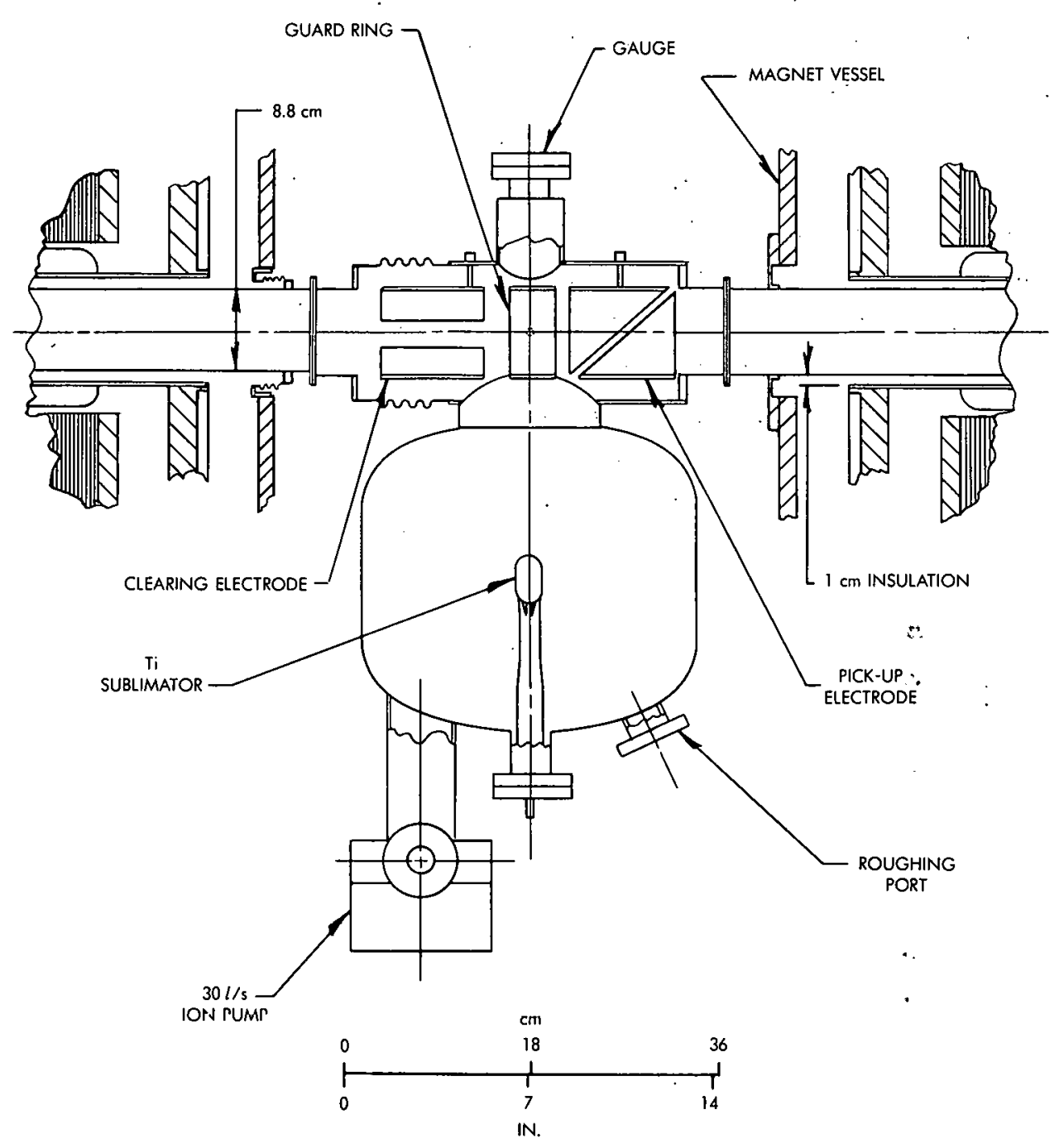

Fig. III.19. Typical ISABELLE vacuum pumping station. 
$1 \times 10^{-11}$ Torr. The ISABELLE pumping station, Fig. III.19, which has an effective pumping speed of $1600 \mathrm{liter} / \mathrm{s}$ and 1000 liter/s for $\mathrm{H}_{2}$ and $\mathrm{Co}$ respectively, reached this pressure within 3 days. The warm bore design includes a dewar construction which allows direct access to all joints and bellows as well as vacuum and beam monitoring equipment. The result is essentially a "conventional" ultrahigh vacuum system. In the insertion regions, however, the experimental physics requirement will undoubtedly dictate special pumping arrangements and chamber configurations (Herrera 1976b, Edwards. 1976). Thus large changes in chamber. diameters will necessitate additional local clearing electrodes in order to eliminate the possibility of "electron traps." 
8. POWER SUPPLIES, INSTRUMENTATION AND CONTROL SYSTEMS

\section{Magnet Power Supply}

The power supply system necessary to energize the windings of the ISABELLE magnets consists of some 458 individually controlled power supplies (see Table 8 ).

The demands on the main field supply are modest due to the slow acceleration cycle. For a total energy of about $400 \mathrm{MJ}$ stored in the bending magnets of each ring, a 4 MVA rated power supply is adequate. With a maximum dipole current of $4.0 \mathrm{kA}$, the required power supply voltage is about $1 \mathrm{kV}$. These parameters are within the range of solid state rectifiers-inverters. The main field power supply will be a twelve-phase SCR system with a passive RLC filter to reduce the ripple to the required tolerance $\Delta I / I \leq 10^{-6}$ at full field.

\section{Table 8. ISABELLE Magnet Power Supply System (2 rings)}

\begin{tabular}{lcc}
\hline \multicolumn{1}{c}{ Description } & $\begin{array}{c}\text { Number } \\
\text { Required }\end{array}$ & Specifications \\
\hline Main dipoles and quads & 2 & $4000 \mathrm{~A}, \pm 0.01 \%(\mathrm{a})$ \\
Low-beta insertion quads & 2 & $4000 \mathrm{~A}, \pm 0.005 \%$ \\
Low-beta insertion quads (warm) & 2 & $1000 \mathrm{~A}, \pm 0.005 \%$ \\
Insertion quad trim & 34 & $\pm 100 \mathrm{~A}, \pm 0.005 \%$ \\
Ring quadrupole trim & 4 & \\
Systematic correction & 16 & $\pm 50 \mathrm{~A}, \pm 0.005 \%$ \\
Equilibrium orbit correction & 96 & 2 \\
Coupling & & \\
& 252 & $\pm 50 \mathrm{~A}, \pm 0.05 \%$ \\
Equilibrium orbit correction & 48 & $\pm 1000 \mathrm{~A}, \pm 0.05 \%$ \\
sew quadrupoles (warm) & & \\
\hline (a) & &
\end{tabular}

(a) $10^{-6}$ during flat top, using feedback from beam position or magnetic field.

The main ring quadrupole magnets are connected in series with the main ring dipoles. Separate windings on the quadrupole magnets, energized from $t 50$ A trim power supplies will provide 
$\pm 4.5 \%$ gradient correction. This arrangement permits a reduction in tolerance requirements for the quadrupole power supplies by an order of magnitude. In general, the insertion quadrupoles will also be energized in series with the main ring dipoles. However, in the low-beta insertion, the quadrupoles Q3 and Q4 will be independently powered with a $1000 \mathrm{~A}$ and a $4000 \mathrm{~A}$ power supply respectively and, will be controlled to a tolerance of \pm 5 parts in $10^{5}$. Trim coils in the insertion quadrupoles will provide a $\pm 9 \%$ gradient correction and will be powered by $\pm 100 \mathrm{~A}$ power supplies. All other field correction windings on the dipoles and the quadrupoles will be energized with independent $\pm 50 \mathrm{~A}$ power supplics.

\section{Beam Instrumentation}

In ISABELLE it will be necessary to maintain two proton beams of small cross-sectional area crossing each other at six intersection regions for periods of many hours while keeping the background radiation far below the desired interaction rate. Instrumentation must therefore be provided to observe those beam characteristics which are of interest to both the experimental and accelerator physicist. Table 9 is a listing of the basic properties of such instrument systems. Beam current, particle momentum, and luminosity are of particular importance to the experimentalist. of these, the luminosity, which is a measure of the beam-beam collision rate, will be determined at each of the six intersections. However, the highest accuracy, $0.5 \%$, will probably be achieved in only one of them where precision total cross section experiments are performed.

The remaining beam monitoring systems presented in the table are primarily beam setup and diagnostic tools for the machine. Thus, the radiation and beam loss system, which essentially consists of many ion chambers distributed around the ring, will continuously monitor the background radiation during the injection, 
Table 9. Beam Instruments

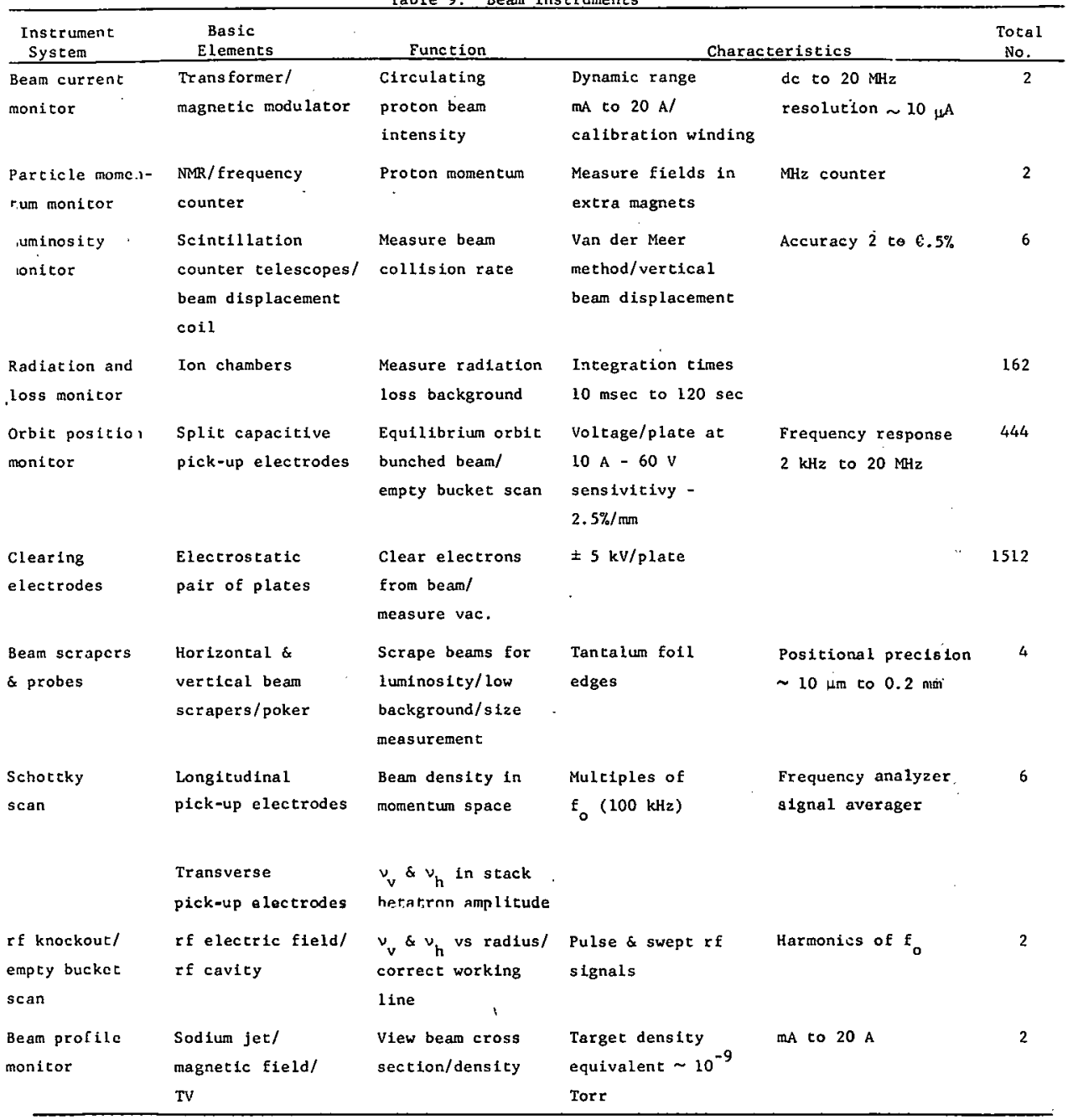


stacking, acceleration and coasting beam phases of the machine operation. Should the radiation become excessive, these radiation monitors will also trigger the beam dump system and thereby prevent damage to the storage ring.

The equilibrium orbits of the two rings will be observed by a set of 444 capacitive pick-up electrodes. This information will be used to correct the orbits by properly powering the dipole correction windings in the machine. It is clear that for a storage ring the central orbits must be stable over long periods of time.

As discussed in the vacuum section of the proposal, the clearlng electrodes are needed in order to establish a low value of neutralization of the circulating proton beams. In addition, it has been found at the CERN ISR (Gröbner 1976) that, since they are particularly sensitive to the ionization of the residual gas, these electrodes can also serve as a very good means of measuring the low vacuum $\left(<10^{-11}\right.$ Tor $r$ ) in the chamber. In this way, it will be practical to observe the vacuum conditions at about a thousand locations in the two ISABELLE rings.

The Schottky scan technique, which has been developed at the CERN ISR (Borer 1974), is a method of measuring the shot noise specțrum at frequencies harmonically related to the particle revolucion frequency in the storage ring. By observing the signal proportional to the total current, one obtains a profile of the proton density versus momentum spread. Similarly, when the signal is derived from a positional sensitive electrode, one can obtain the horizontal and vertical tune values and thereby derive the working line of the beam stack. Since this type of observatio.. does not interfere destructively with the beam, it should be an important diagnostic tool for ISABELLE.

Beam scrapers and probes have a number of functions in a storage ring (Bryant 1973). By positioning them such that a 
change in beam intensity is observed, one can evidently determine the spatial limits of the beam in both the radial and vertical planes. In addition, such scraping can be used to decrease the halo of particles surrounding the more dense central core of the beam and, as a consequence, reduce the radiation background in the ring. At the CERN ISR, vertical scraping is also employed to decrease the effective height of the stack with a resultant increase in luminosity. In Table 9 we have included the precision horizontal and vertical scrapers required. Additional flipping targets and fixed collimators will be necessary, but these do not have to be precision units.

The physical profile of the beam due to betatron oscillations and momentum spread, that is, its density distribution in a plane transverse to its direction of motion, is difficult to observe, particularly if the observation is not to interfere with the beam. The sodium curtain has been developèd at CERN (Vosicki 1975) for this specific purpose. By allowing the electrons, produced by the beam crossing a sodium vapor jet, to impinge on a fluorescent screen, a two-dimensional profile of the proton distribution can be viewed on TV. Such a picture of the beam density and location is clearly helpful in the operation of the machine.

\section{Control System}

There are various functions that the control system of ISABELLE must perform. These functions include the operation of the machine, modification of operating parameters and pronedures, monitoring of parameters of interest, machine and enironmental protection, and an array of support functions (bookkeeping, program development, etc.) (Dimmler 1977, Mallory 1975a). In line with the experience of other large accelerator control systems, under construction or operating (PEP 1974 Proposal, McDaniel 1975, Crowley-Milling 1975, Mallory 1975b, Daniels 1973), the control system of ISABELLE will be built around a network of computers (Fig. III.20). 


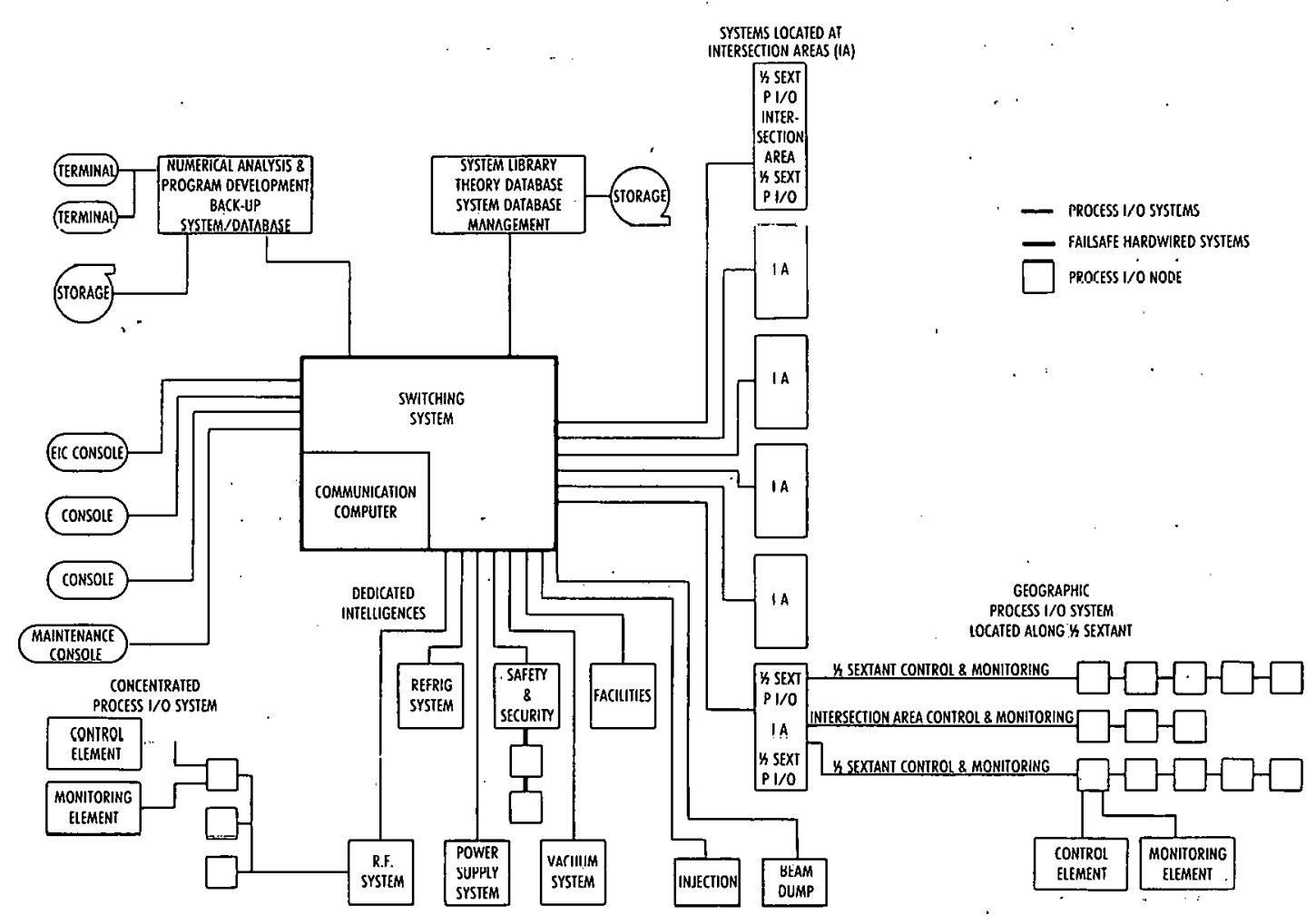

Fig. III.20. Block diagram of the overa11 contro1 system for ISABELLE. 
The data base subsystem will contain a complete record of the ISABELLE status. In addition, it will contain both reference data and programs. This will be a a relatively sophisticated midicomputer incorporating large scale memory, large capacity disk, and other peripherals. The requirement of reliability dictates that two of these elements be incorporated. When it is not required as a back-up, the second system is available for program development and numerical analysis.

The general controls subsystem supports the human factors interface. This is the means by which operators, designers, and experimenters communicate with the control system. The main control room will contain three control consoles with a fourth, nominally dedicated to maintenance, located elsewhere in the service building. These consoles are driven by minicomputers and include graphics displays, cursor manipulation, touch panels, etc. Design of the control room consoles is an important aspect, since comprehensive monitor displays and convenient operator interactions are imperative.

The eight computers which have specific names, ( $r f$, vacuum, refrigeration, etc.) should be considered as dedicated intelligence: they manage their particular subsystem. The particular subsystem of interest may be connected locally to the dedicated intelligence (rf, for example) or it may be distributed geographically at some distance (e.g., vacuum), or it may be a combination of the two (refrigeration). A dedicated intelligence has access to a particular concentrated process I/O system for control and monitoring : local process variables. It also communicates with the necessary irtions of the geographic process I/O network via a message system. These dedicated intelligences have local peripherals and can operate independently of the control computer network, if necessary. For example, if the message system is not available, the cryogenic refrigerator could still be monitored and controlled. The design takes full advantage of the inherent parallelism available in computer networks. 
The message subsystem is needed to effect communication between the various ISABELLE control elements. Following the SPS (Crowley-Milling 1975) example, the message system includes a computer which functions as a switching system and communications supervisor. It makes connections between computers and oversees the message traffic. This allows heavily loaded message links to be pinpointed, and general diagnostics of the message system to be performed.

In order to avoid undue complexity in the message subsystem and a subsequent decline in performance and reliability, a level of nonintelligent process I/O packages is introduced. The process I/O networks are of two kinds. There are local clustered systems where the process variable density is high and it is cost effective to have a process I/O package dedicated to a single function. On the other hand where the process variable density for a single system is low a multifunction process I/O package geographically distributed around the ring is used. The use of such I/O units and a network avoids the deterioration of the analog signals over long distances.

One would like the computer communication medium and the process I/O communication medium to be conceptually compatible and convertible. It this feature could be built into the system, it would be possible for computers to have their functions performed by other computers without physically moving them. This would be very useful from the point of view of program development and rellability. IEEE standard number 488 (the General Purpose lnterface Bus) may be the basis for the $1 / U$ communication medium.

It is anticipated that microprocessors will be used on a large scale for what one might call "intelligent instrumentation." There are approximately 30,000 monitor and control points associated with ISABELLE itself (see Table 10). Many of these variables can be locally processed. An intelligent instrument could monitor 
such variables and only communicate with the network when the variables exceed preset limits. Other possible applications are in the field of beam instrumentation. Such instrumentation will significantly reduce the data flow over long distances.

Tablè 10. Monitor and Control Variables in ISABELLE Rings

\begin{tabular}{|c|c|c|c|}
\hline Function & $\begin{array}{l}\text { No. of } \\
\text { Variables }\end{array}$ & $\begin{aligned} \text { Sample or } \\
\text { Response Time }\end{aligned}$ & Comments \\
\hline \multirow[b]{2}{*}{ Coil voltages - dipoles } & \multicolumn{3}{|c|}{ Monitor Variables } \\
\hline & 2928 & $10 \mathrm{msec}$ & Includes correction coils \\
\hline Coil voltages - quadrupole & Les 2712 & $10 \mathrm{msec}$ & Includes correction coils \\
\hline Magnetoresistance probes & 1464 & $10 \mathrm{msec}$ & $\begin{array}{l}\text { Sextupole \& dipole fields } \\
\text { in all dipoles }\end{array}$ \\
\hline Helium pressure \& temp. & 2140 & $1 \mathrm{sec}$ & Each magnet \\
\hline Dewar vacuum & 336 & $1 \mathrm{sec}$ & \\
\hline U1trahigh vacuun gauges & 1536 & $1 \mathrm{sec}$ & \\
\hline Mass spectrometer & 336 & $100 \mathrm{msec}$ & \\
\hline Ion pumps & 1176 & $1 \mathrm{sec}$ & \\
\hline rf system & 200 & $0.1-10 \mathrm{sec}$ & \\
\hline Beam instrumentation & $\sim 5000$ & & See Table 9 \\
\hline Injection \& ejection & $\sim 1500$ & & \\
\hline Helium refrigeration & $\sim 500$ & & \\
\hline & & ontrol Variab & les \\
\hline Magnet Power Supplies & $\begin{array}{r}458 \\
2000\end{array}$ & & $\begin{array}{l}\text { Programmable current } \\
\text { Binary status control }\end{array}$ \\
\hline Sector valves & 84 & & Binary status control \\
\hline Sputter Ion Pumps & 1176 & & Binary status control \\
\hline Titanium Getter Pumps & 1536 & & 3 states - off, flash, outgas \\
\hline Bakeout Heaters & 3708 & & Binary status control \\
\hline Turbopumps & 42 & & Billary slatus control \\
\hline rf System & 10 & & $\begin{array}{l}\text { Programmable phase, voltage, } \\
\text { loop gain }\end{array}$ \\
\hline & 100 & & Binary status control \\
\hline Helium Flow & 12 & & Programmable \\
\hline
\end{tabular}


Inherent in any system architecture is the concept of hardware/software trade, i.e., the proper selection of hardware and software elements so that an optimal configuration can be achieved. The proposed separated function architecture emphasizes the use of hardware (which has shown and continues to show a rapidly decreasing price/performance ratio) while effort has been made to limit system software, clearly a labor intensive area. Added advantages of this approach are the ease with which subsystems may be independently developed, more rapid maintenance, and the possibility of bypassing inoperative hardware for continuous accelerator operation. 


\section{PHYSICAL PLANT AND EXPERIMENTAL HALLS}

\section{Location of ISABELLE}

The location of ISABELLE on the Brookhaven site, as shown in Fig. I.2, was determined by beam availability from the AGS, physical characteristics of the site topography, relationship of the site boundaries to the proposed ring, proximity of existing site utility systems and roadway networks, and finally the overall impact on the existing ecology and the natural environment. As presently rroposed, the siting of ISABELLE has considered all of the above in a manner consistent with sound engineering judgment and cost effective analysis. The location of the beam transfer line to the ISABELLE ring effectively uses the existing H-10 beam tunnel, and preserves those experimental areas presently in use. The extension of this beam line to the ISABELLE $r i n g$ is both convenient and economical from the construction viewpoint. The siting of the main ISABELLE ring recognizes the need for adequate radiation protection and satisfies this requirement by providing adequate distances from the west and north site boundaries. By coupling the major facilities of ISABELLE to the existing AGS services, the utility system, and access roadways necessary to support the new facility were developed in a prudent, cost conscious and effective manner. A computer assisted analysis of all earthwork ("cut and $f i l 1 "$ ) has optimized final elevations of magnet tunnel and the perimeter roadway, resulting in minimum disturbance to the natural surroundings of the proposed site. Such considerations are discussed in the Conventional Facilities nnsign Report (Ammann \& Whitney 1977).

\section{Magnet and Beam Transfer Enclosures}

A cross section of the magnet enclosure is shown in Fig. III.2. The enclosure, which has a total length of $3834 \mathrm{~m}$, will be constructed of steel multiplate arch approximately $4.6 \mathrm{~m}$ wide by $3.1 \mathrm{~m}$ high. It will be covered with $4.0 \mathrm{~m}$ of earth for radiation 
shielding, to maintain external radiation below tolerance levels. The thickness of $4.0 \mathrm{~m}$ is indicated by experience from Fermilab and by Monte Carlo simulation calculations. To provide a conservative safety factor, the enclosure will be designed to permit a later increase to $6 \mathrm{~m}$ shielding thickness if operating experience should show a need for it. There is also a need to absorb muons that fan out approximately tangentially in the median plane. This need is filled automatically if the beam height is sufficiently below grade level. In a large fraction of the ring, however, the grade level is low enough that a muon shield is needed around the exterior of the magnet enclosure. The radial width of the shield will vary from $15 \mathrm{~m}$ to a maximum of $120 \mathrm{~m}$ at one point, with a height $4.0 \mathrm{~m}$ above beam elevation. At the beam dump and scraper locations the radiation shielding must be thicker than over the normal magnet $r$ ing enclosure because most protons interact there. The thickness will be $6.7 \mathrm{~m}$ with strength adequate for increase to $8.5 \mathrm{~m}$.

Access to the magnet enclosure will be from the experimental regions approximately every $640 \mathrm{~m}$. Emergency exit will be through perpendicular tunnels located throughout the $r$ ing at intervals of approximately $90 \mathrm{~m}$. Equipment bays will be provided to house support services for the machine at these locations.

The foundation for the magnet enclosure will be a composite footing and slab-on-grade of reinforced concrete. Subsurface investigations (test borings) have shown that the subsoil around the ISABELLE site has a presumptive maximum bearing in excess of $59,000 \mathrm{~kg} / \mathrm{m}^{2}$, which indicates the simple slab foundation for the ring magnet will be entirely satistactory.

The beam transport enclosure will be constructed similarly to the main magnet enclosure but somewhat less in cross-sectional area. Interior dimensions will be approximately $2.5 \mathrm{~m}$ wide by $2.5 \mathrm{~m} \mathrm{high}$ and embanked by $3.5 \mathrm{~m}$ of earth for radiation shielding. The total length of the straight section, transition, and the two branches will be about $630 \mathrm{~m}$. 
At each of the six experimental regions a building of approximately $140 \mathrm{~m}^{2}$ will be provided to house support services for those areas. These buildings will provide space for service equipment and rest rooms for the experimental halls and limited areas for experimenter's use. Located in the vicinity of the beam transport area, a support building of approximately $460 \mathrm{~m}^{2}$ will be constructed and generally serve similar functions needed along the beam transport enclosure.

\section{ISABELLE Service Building}

At a location adjacent to the $50^{\prime}$ clock insertion and to the main magnet enclosure a large operational service area will be constructed. The area is an integrated functional complex of buildings housing the following main facilities: 1) service building with access tunnels; 2) electrical substation; 3) helium compressor and utility compressor structure; 4) helium storage yard; 5) access roads, circulation roads and pavements; 6) parking areas; 7) sidewalks; 8) cooling towers.

The service building will be developed as three separate but interrelated wings covering a total of $4500 \mathrm{~m}^{2}$, each housing particular grouped functions. The three wings are: a) the accelerator control wing; b) the support wing, and c) the cryogenic equipment wing. The building is connected to the magnet enclosure by personnel utility access tunnels at both the cryogenic equipment and support wings. The designs of the wings will differ substantially because of the differences in function. ne control wing is to be a steel frame and masonry/concrete structure providing electronic controls and convenient adjacent office and work space. The support wing constitutes an industrial area of relatively high ceilings housing the shops and equipment necessary to maintain the functioning facility. The cryogenic wing will be a high bay area supporting the main helium refrigeration equipment. This wing will communicate with an adjacent 
outdoor area in which the helium compressors are to be located below grade.

\section{Roads and Utilities}

Access to the proposed location of the main ring and the injection transport tunnel utilizes the present roadway systems without major alteration or disruption to the existing operations. The new road network to ISABELLE is basically an extension of the present system serving the AGS. From the east approach, Thompson Road and Railroad Street will be extended to the location of the service building and thence the main ring. From the west approach a new spur will connect Upton Road to the ISABELLE ring road. The perimeter or ring road will interconnect each of the experimental regions as well as the access roads previously mentioned (Fig. III.1).

Domestic water, sanitary system, steam \& condensate, telephone and fire alarm and the primary electric service will be provided from the present utility networks which serve the AGS. These systems are capable of extension and/or expansion with a minimum of alteration or modification. For the most part, ample capacity exists in such services as water, sanitary, steam \& condensate to allow a simple extension of these facilities to the ISABELLE site. In the case of the primary power, a 50\% expansion of the present substation (AGS) is feasible and practical to provide the required service for the accelerator systems and experimental needs; however, new ductbanks and cable are required.

\section{Experimenta1 Areas}

There will be four enclosed halls and two free areas so that all six intersection regions can be used for experimental purposes. A great variety of experiments will be possible at ISABELLE, with widely varying requirements. Experiments concerned with particles of small transverse momentum require transverse dimensions not significantly greater than those of the magnet enclosure. Experiments 


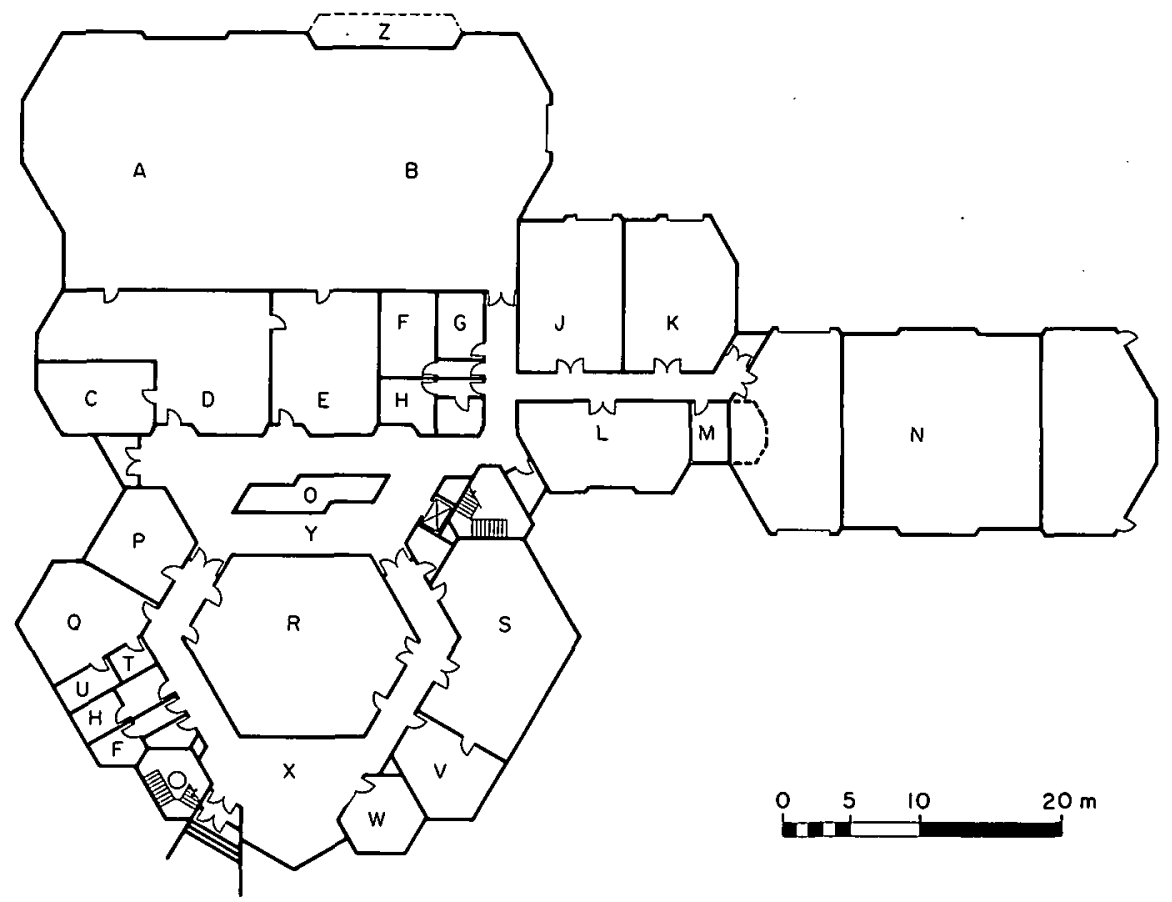

Fig. III.21. Plan view of the first floor of the service building which houses refrigeration and vacuum equipment, control room and computer, and other functions necessary for ISABELLE operation. Legend:

A - ejection dump equipment area; B - rf and power supply equipment areas; $C$ - computer support;

D - computer room; E - low level rf and instrumentation lab; F - mens toilet, locker and shower facilities; $G$ - lunch room; H - womens toilet, locker and shower facilities; J - vacuum equipment and $1 \mathrm{ab}$; $\mathrm{K}$ - shop; $\mathrm{L}$ - parts and tool room; $\mathrm{M}$ - cryogenic equipment control room; $N$ - cryogenic equipment area; $P$ - computer users room; $Q$ - ready room; $R$ - main control room; S - electronics laboratory; $\mathrm{T}$ - toilet; $U$ - kitchenette; $V$ - operations office; $W$ - office; $\mathrm{X}$ - lobby; Y - atrium; Z - rf substation. 
concerned with particles of high transverse momentum, whether leptons, hadrons, or perhaps still other types, will require substantial width and height. Some experiments require only small detectors, while others need large detector systems and analyzing magnets. There are four experimental halls, two suitable for large detectors, a wide hall, and a narrow hall, with locations and dimensions shown in Table 11. In each case the tunnel adjacent to the experimental hall will be enlarged to $6.0 \mathrm{~m}$ width up to approximately $90 \mathrm{~m}$ from the crossing point.

Table 11. Dimensions of Enclosed Experimental Halls

\begin{tabular}{lccccc}
\hline Location & $\begin{array}{c}\text { Length } \\
(\mathrm{m})\end{array}$ & $\begin{array}{c}\text { Width } \\
(\mathrm{m})\end{array}$ & $\begin{array}{c}\text { Height } \\
(\mathrm{m})\end{array}$ & $\begin{array}{c}\text { Depth Below } \\
\text { Beam } \\
(\mathrm{m})\end{array}$ & $\begin{array}{c}\text { Crane } \\
\text { Capacity } \\
\text { (tons) }\end{array}$ \\
\hline One O'clock & 80 & 8 & 6 & 1.75 & 10 \\
Five O'clock & 16 & 42 & 12 & 3 & 40 \\
Seven o'clock & 58 & 18 & 15 & 5 & 40 \\
Eleven o'clock & 58 & 18 & 16 & 6 & 40 \\
\hline
\end{tabular}

The design concept for experimental halls is based on a cellular construction, with walls of hollow concrete boxes filled with gravel to attain the required shielding thickness. The roof is of concrete beam construction covered initially with 2.1 to $2.7 \mathrm{~m}$ of earth, but capable of supporting 4.0 to $4.5 \mathrm{~m}$. Footings and floor slabs are conventional reinforced concrete construction. Cable trays for, instrumentation and control circuits as well as ordinary building services (heat, light, and power) will be installed. Direct truck access will be provided through concrete shield doors for bringing in experimental equipment and accelerator components and removing them when needed. A combination personnel and utility tunnel will connect each hall with its support building.

An open hardstand approximately $30 \mathrm{~m}$ by $45 \mathrm{~m}$ will be provided at all enclosed halls. Trailers or temporary buildings for experimenters' use will be located on it or adjacent to it. Typical 
experimental halls are shown schematically in Figs. III.22a, III.22b and III.22c with details of access tunnels omitted.

The two free areas will consist of hardstands $80 \mathrm{~m}$ in length along the beam and $40 \mathrm{~m}$ in width perpendicular to it, $3.0 \mathrm{~m}$ below the beam line. At the ends concrete retaining walls will extend out from the tunnel. Movable concrete shielding blocks will be used to enclose the beam and any experimental equipment that is in place between the retaining walls. An appropriate weatherproof covering will also be provided. Most concrete blocks will be available from BNL inventory. This will provide flexibility to rearrange shielding and experimental setups in accordance with the future needs of the experimental program. Figure III.22d shows the possible appearance of a free area. Eventually these areas might be enclosed with permanent structures, but this proposal does not provide for doing so. 
QUADRUFOLE DOUBLE Q1 AND Q2

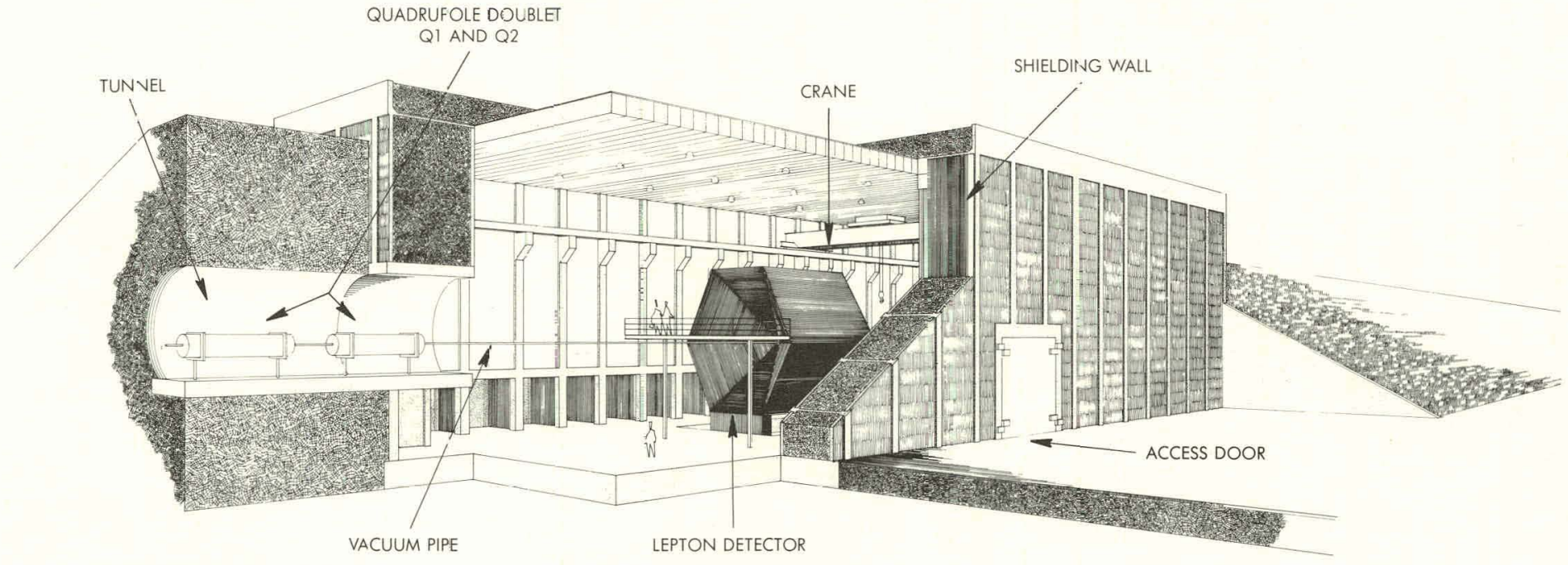

Fig. III.22a. Schematic view of the experimenzal hall located at the "e-even $0^{\prime}$ 'clock insertion" as seen from inside the ring. The cut-avay view shows the experimental space occupied by a large, hexagonally shaped -epton detector. 


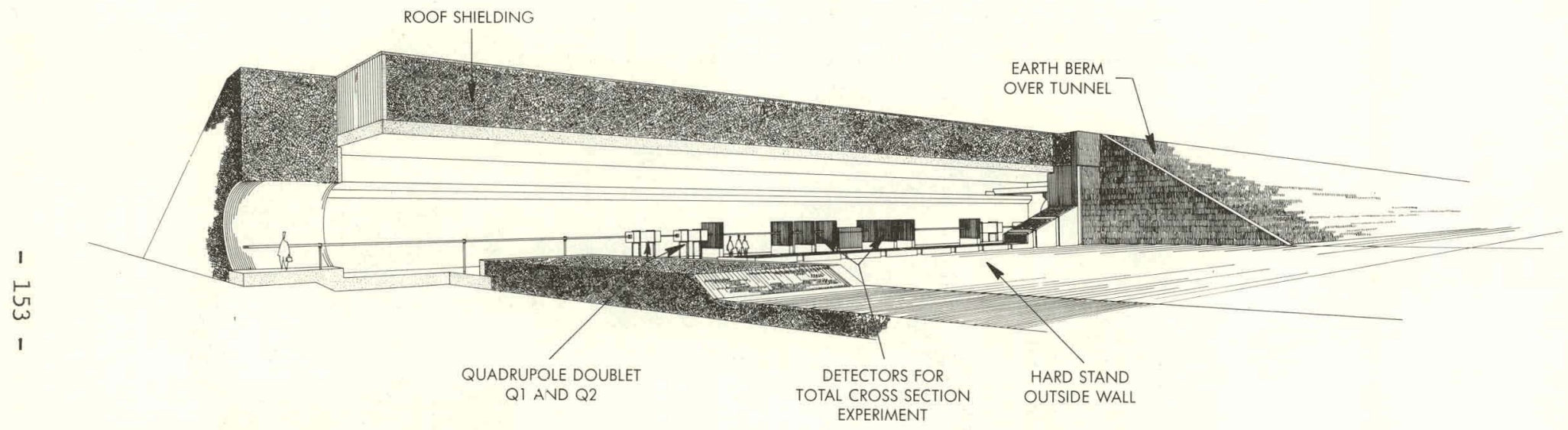

Fig. III.22b. Schematic view of the experimental hall at the "one o'clock insertion" as seen from inside the ring. The cut-away

view shows typical equipment for measuring the $p-p$ total

cross section located on both sides of the beam intersection

region. 
QUADRUPOLE DOLBLET

Q1 AND Q2

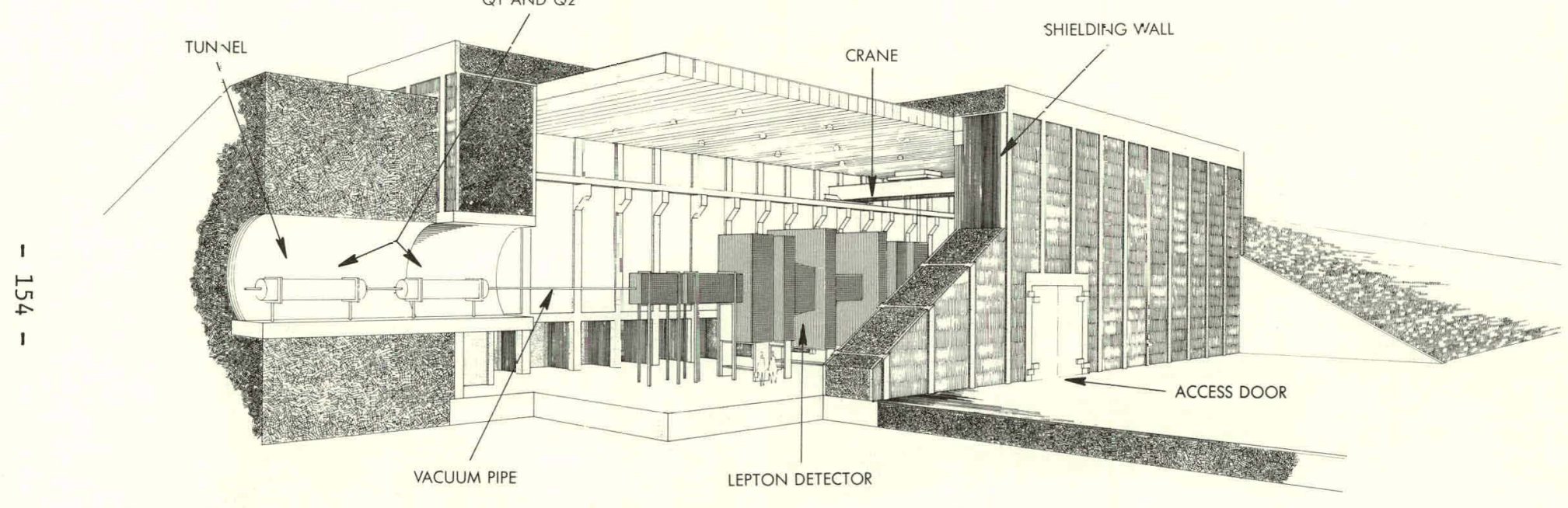

Fig. III.22c. Schematic view of the experimenta1 hall located at the

"seven o'clock insertion". The cut-away view shows

a large aperture spectrometer, labelled "hadron detector". 


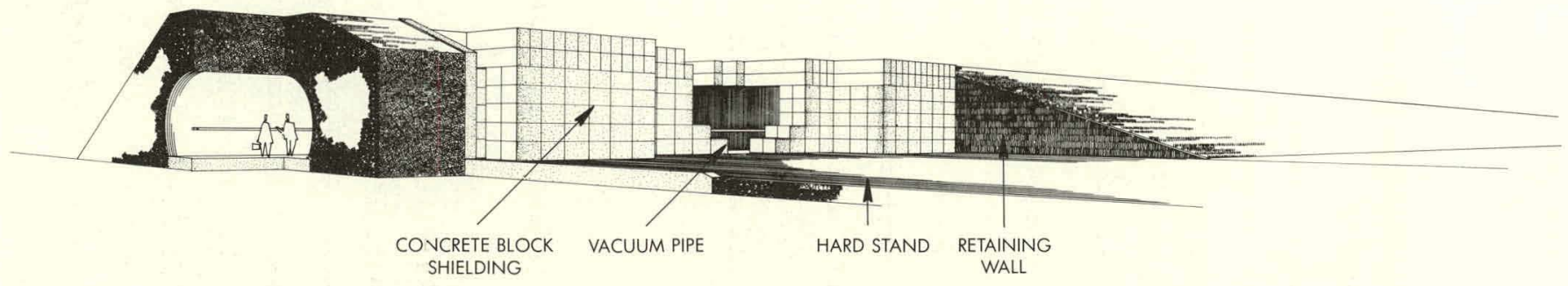

Fig. III.22d. Schematic view of the free area at the "three o'clock insertion" as seen from inside the ring. Concrete block shielding is shown enclosing the intersection, with a cut-away view depicting

the vacuum pipes. 
The storage rings will be expected to operate for extended periods of time reliably and safely. Because of these objectives, special care will be taken with the design and construction of the facility. Prototype units will be tested thoroughly so that accurate and sensible specifications can be written for the technical components. With inspection and testing it will be possible to assure delivery of satisfactory components.

The conventional facilities and experimental areas will have design criteria specified to provide a safe environment for people as well as apparatus. During operation all accelerator and research personnel will be excluded from the tunnels and research halls at ISABELLE. The earth shielding that surrounds the beam enclosure is sized to reduce the background radiation in areas occupied by experimental and accelerator physicists to acceptable levels. In addition, site access during operation will be controlled so that laboratory visitors are protected. Shielding will be added in selected areas around the storage ring so that there will be negligible radiation problems for the general public at the site boundary.

It is expected that the facility will have extended periods of operation interrupted periodically for an hour or so every day to provide experimenters with opportunities to install or adjust their apparatus. Access to the beam tunnels will be controlled so that only the experimental halls must be cleared before operation resumes. Every four or five months the entire facility will be closed down for maintenance and extensive modification. After checking for areas of residual radiation, the tunnel and halls will be available for general work. At the end of these periods, the whole facility will be "searched and secured" before resuming operation. 
During operation the entrances to all the radiation areas will be locked. Interlocks will be attached to the doors of all access points so that the proton beams will be shut down if the interlock chain is broken. Appropriate administrative controls will be exercised so that only qualified experimenters and accelerator personnel have access to the beam regions. These individuals will be required to wear personal radiation monitoring devices such as film badges or dosimeters while working in radiation areas.

Instrumentation will be located around the rings to detect signs of beam misbehavior if that should arise. At such an occurrence, the beams will be ejected from the rings into an external beam dump away from occupied areas. It is expected that this will be an infrequent occurrence, yet a necessary precaution to protect people and equipment.

Finally, the ring is located such that road access for emergency equipment is straightforward. If required, fire and other emergency vehicles can come from their stations by clear road to the service buildings and experimental halls. 


\section{COST ESTIMATE AND SCHEDULE}

\section{COST ESTIMATE}

The cost estimate, critical milestones, schedule and machine operating requirements are presented in this section.

We have been extremely fortunate in having available Construction Planning and Design (CP\&D) funds. This money was allocated by DOE to "provide cost estimates based on sufficient study to be as reliable as possible." To this end the design of ISABELLE was brought to a stage where critical advice, design considerations and independent review of complete systems could be made by outside consultants and industrial firms. In the estimate shown in Table 12 an asterisk has been placed next to those systems which have had CP\&D funds used in reviewing major elements of that system or the entire system.

In preparing the cost estimate each component group provided, in as great detail as possible for that system, the cost of equipment, personnel requirements and time schedule for all phases of design and construction. The pricing accuracy of all aspects of the estimate was greatly improved because of the active participation by our consultants and industry. The documentation thus assembled by the component group was presented to the "Finance Committee" for an initial review and critique and then a final review prior to acceptance. At this point the accelerator component costs were tabulated. From the personnel figures submitted, manpower for assembly and testing was separated and costed with the component. Al1 other personnel were costed in the Engineering Design Inspection and Administration (EDIA) category. Thus for EDIA and assembly and test 1630 man-years effort through completion of construction is required.

The Architect-Engineering-Management (AEM) cost was set at $16 \%$ of the cost of conventional facilities. This percentage is within the limits recommended by construction advisory services. 
We have included contingency funds in the estimate as follows: Accelerator systems :

$\begin{array}{lr}\text { (variables for individual systems) } & 15-20 \% \\ \text { Facilities } & 15 \% \\ \text { EDIA } & 20 \% \\ \text { AEM } & 15 \%\end{array}$

The estimate is in 1977 dollars and the escalation shown is within the guidelines suggested by ERDA. The percentages are FY 77 - 79 salary and wages, $\mathrm{A} / \mathrm{E}$, and conventional facilities at $7 \%$ per year and machine components at $6 \%$ per year. For the years thereafter all elements are escalated $6 \%$ per year.

\section{CRITICAL MILESTONES AND SCHEDULE}

An overall schedule for the ISABELLE Project is shown in Fig. IV.1. It is based on the following projected obligation authority (BNL, Schedule 44, 1977):

\begin{tabular}{|c|c|c|c|c|c|c|c|}
\hline & EY78 & EY79 & EY80 & FY81 & FY82 & EY83 & FY84 \\
\hline$(\$ 1000)$ & $1,750(C P \& D)$ & 80,000 & 82,000 & 29,000 & 20,000 & 16,000 & 11,000 \\
\hline
\end{tabular}

Target dates are as follows:

Project Authorized

October 1978

Start Detailed Design of Buildings and Technical Componente

October 1978

Start Clearing of Site

January 1979

Start Procurement of Accelerator

Components

January 1979

Start of Component Delivery

October 1979

lagnet Assemb1y Commences

June 1980

Partial Occupancy of Magnet Enclosure

June 1980

Beneficial Occupancy of Buildings and

Start of Installation of Accelerator

Components

January 1981

Ready to Start Beam Injection and Testing

October 1983

Complete Construction - Accelerator Ready

for HEP 


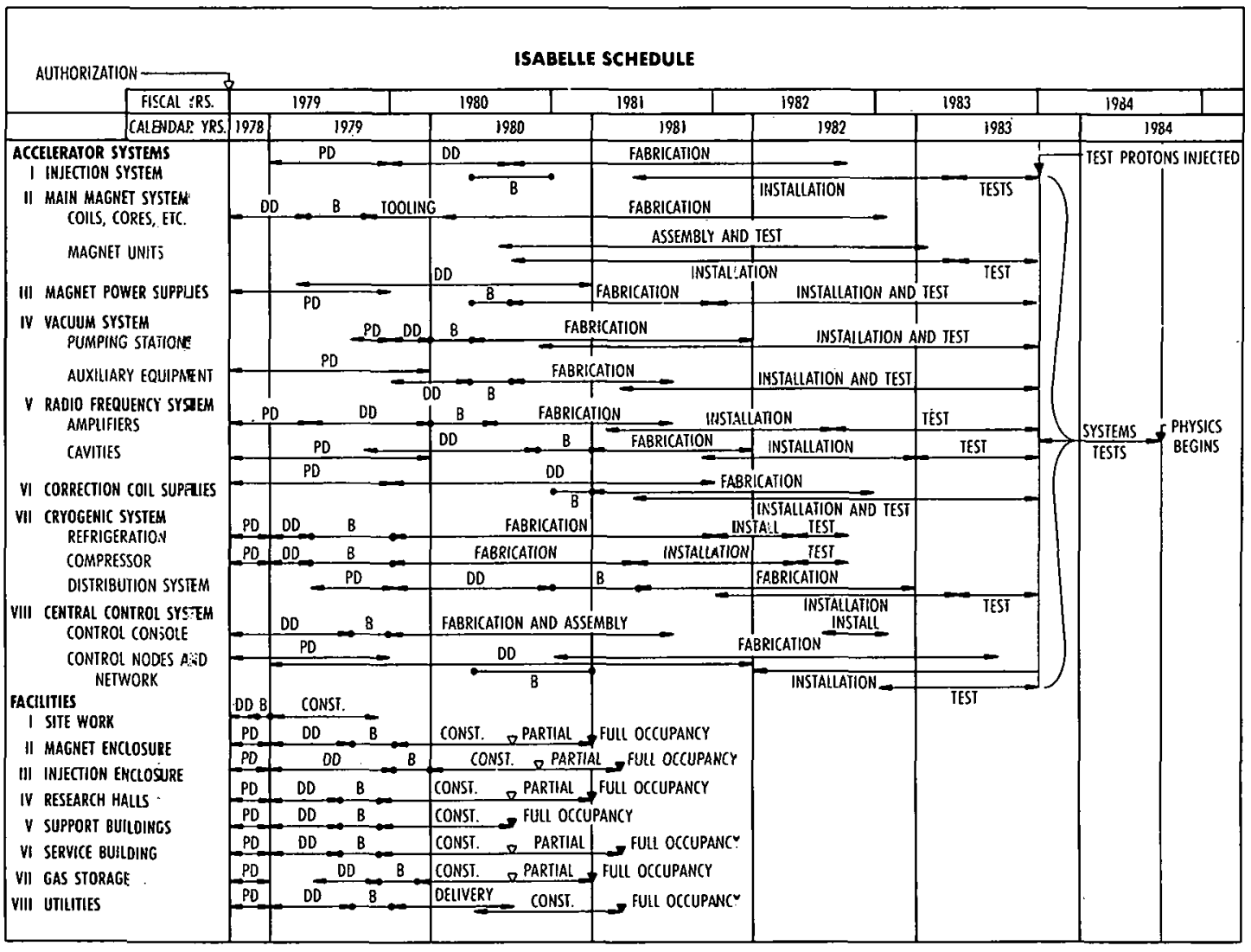

Fig. Ii.1. Overall schedule for construction and testing of ISABELLE to the beginning of the high energy physics research. The following abbreviations are used: PD - preliminary design, DD - detailed design, B - bid. 
A. Accelerator Systems

I. Injection system 5,410

Magnets and power

supplies

3,080

Vacuum system

Collimator, beam

stop

Services

Controls \& instrumentation

Labor \& miscellaneous

$63.251,390$

II. Main magnet system ${ }^{*}$

Magnets, supports,

installation

47,560

Vacuum system and

power leads

1,660

Beam Dump

3,200

Fault protection

Labor \& miscellaneous

$490.25 \quad 10,780$

III. Magnet power supply

1,750

Power supply \& cable

900

Controls

250

Labor \& Miscellaneous

27.0

600

IV. Vacuum system ${ }^{*}$

8,360

Bore tube, pumps, power

supplies, valves

3,670

Bake-out system

Pick-up and clearing

electrodes

Labor \& miscellaneous 145.0

3,110 
Table 12 (continued). Summary of Cost Estimate (Dollars in thousands)

Man-years

Total

V. RF System ${ }^{*}$

Stacking system -

power amplifiers \&

cavities

800

Accelerating system -

power amplifiers \&

cavities

2,200

Controls

400

Labor \& misce1laneous

$77.5 \quad 1,800$

VI. Beam monitoring \&

correction controls

5,200

Beam monitoring \&

instrumentation

1,310

Correction coil power supp 1y

2,430

Labor \& miscellaneous

$$
43.0 \quad 950
$$

VII. Refrigeration system

compressor etation

4,000

Refrigeration station

4,500

Ambielit tenperalure

piping \& gas stiriage

plus gas

1,500

Low temperature piping

2,000

Controls

500

Labor \& miscellaneous $155.0 \quad 3,280$

VIII. Central control system"

$*$

Control console and computer

1,010

Remote control nodes \&

cable lines

4,450

Security systems

300

Labor \& miscellaneous

50.0

1,230

15,780

4,690

6,990 
Table 12 (continued). Summary of Cost Estimate (Dollars in thousands)

B. Special equipment

I. Laboratory test instruments

II. Vehicle 20

C. Facilities ${ }^{*}$

I. Land improvements

1,030

Storm drainage

470

Roads \& paved areas

Fencing \& seeding

II. Magnet enclosure

clearing, excavation, backfi11

3,430

Tunnel structure

6,200

Mechanical services

960

Electrical services

III. Beam injection tunne1

1,110

Tunnel structure

Modification to $\mathrm{H}-10$

tunne 1

Mechanical services

Electrical services

IV. Experimental areas

8,300

Large detector halls (2)

4,190

Large detector hall

(1)

1,560

Small angle hall

1,240

Open areas (2)

1,310

V. Support buildings

Experimental areas

Injection area (1) 
Table 12 (continued). Summary of Cost Estimate (Dollars in thous ands)

VI. ISABELLE service building

5,330

Structure

2,520

Mechanical services

Electrical services

1,890

VII. Gas Storage

Structure

VIII. Utilities

$$
2,680
$$

Domestic water

Sanitary sewer

Steam \& condensate

Electrica1 - $69 \mathrm{kV}$ primary

Electrical - $13.8 \mathrm{kV}$ secondary

Telephone \& fire alarm

D. Architect engineer (@ 16\%)

of facilities

E. Englnccring, design,

inspection, administrative

(579.0 manyears)

Subtota1

F. Contingency - facilities

(a) $15 \%$; accelerator systems

(a 15-20\%; EDIA @ $20 \%$; A/E @ $15 \% \cdot\} 16.7 \%$

G. Escalation 1977-1979 S\&W, A/E, conv. fac. (a $14.5 \%$, machine

comp. @ $6 \%$ per year; 1979 thru 1984 - 6\% per year

H. Construction Planning and Design Funds (CP\&D) 


\section{ANTICIPATED EQUIPMENT AND OPERATING NEEDS}

\section{Equipment for Experimenters}

A general estimate has been made of the cost of equipment needed for experiments such as those described in Section V. 3 . At this early stage the design of the experimental equipment is known, however, in much less detail than the design of ISABELLE itself, and the cost estimate is correspondingly to be considered preliminary and approximate. The possible experimental program described in section V.3 provides for some major pieces of experimental equipment to be in place and ready for operation at the time of ISABELLE turn-on. Consequently capital expenditures for the equipment begin four years before turn-on and the majority of the cost is incurred before the start of the physics program. This represents a vigorous approach aimed at extensive experimental use of ISABELLE as soon as practical. On the other hand, a continued growth in complexity of the experiments is assumed during the initial years of operation. Thus, the overall costs for the equipment to start the experimental program are spaced over a seven-year period from FY 80 to FY 86.

This represents a projection many years into the future from the time of preparation of this proposal. It is not a plan for the research program during that period, nor for the equipment to carry it out. It is only an estimate to indicate the scope of equipment requirements, made as realistically as possible with a view of both physics objectives and the ability of the ISABELLE and its staff to support the research program.

Research at ISABELLE will be carried out mainly by university zesearch groups, as has been the case at the AGS and before that at the Cosmotron. A considerable amount of equipment will be built

at universities and brought to Brookhaven to carry out experiments. The largest components will undoubtedly be built at Brookhaven, however, as will a number of items of general usefulness in a 
succession of experiments. There are, however, many reasons for university groups to play a substantial role in the construction of all types of experimental equipment, and it is expected that they will do so. Some of the capital equipment costs would therefore be incurred under university auspices, but it is not possible to estimate now what the fraction should be. Accordingly the estimates given here are for the total program cost, without a breakdown into Brookhaven and university fractions.

The estimate of capital cost of equipment required to implement such an experimental program is shown in Fig. IV.2 as a function of time. This is an estimate of the funds required for equipment for a highly productive research program of realistic scope and schedule. The curve includes an allowance for the continuing need for various types of capital equipment for experiments, but not the smaller amount needed for ISABELLE operation and accelerator research. Labor costs are included and the same escalation factors are used as for the ISABELLE cost estimate.

During the period from FY 80 to FY 86 the cumulative cost of equipment to implement the experimental program and cover its continuing needs for capital is $\$ 53,000,000$. These figures do not include any provision for second-generation experimental equipment, since there is no way to estimate that at present. It would be reasonable to suppose, however, that by FY 87 some major new equipment would be proposed that would require an increase in the rate of equipment spending.

\section{Operating Personnel}

To give an indication of the magnitude of effort involved in the experimental program based at ISABELLE an estimate is given for the number of operating personnel required for full-scale utilization. This includes the operation of the AGS to inject protons into ISABELLE and also to provide test beams needed to check out equipment for use at ISABELLE. It also includes limited 


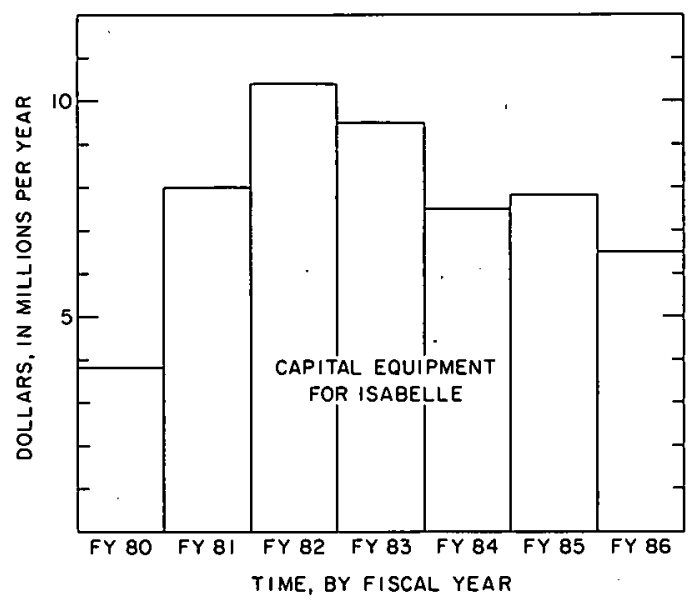

Fig. IV.2. Capital cost per year needed for ISABELLE experimental program according to present estimate, to FY 1986. 
support staff for two large detector facilities, assumed to be equivalent to the Lepton Detector and the Large Aperture Spectrometer. In other areas experimental support will be provided for installation and removal of large equipment, service for major magnets and power supplies, provision of standardized electronics, and program coordination. Thus the operating staff is consistent with the scope and schedule of the projected research program and the equipment required for its implementation. People doing fu11-time particle physics research are not included. The estimate is only for ISABELLE operation and does not include any provision for a research program with conventional AGS beams, which would require additional operating staff.

The projected experimental program begins on a smal1 scale immediately after turn-on of ISABELLE, and essentially full-scale utilization of its capabilities is reached within two years time. The required operating staff is then estimated to be:

$\begin{array}{lr}\text { Scientists } & 53 \\ \text { Professional } & 90 \\ \text { Technical } & 315 \\ \text { Total } & 458\end{array}$

The overall high-energy physics effort would include members of research groups at Brookhaven and from many other institutions, as well as the above ISABELLE operation staff. The number of physicists involved in the research program is estimated to be 200 to 300 , primarily from universities. These research groups will probably also have a considerable number of engineers and technicians working with them in the conduct of their experiments. 


\section{APPEITIXIX}

Table 13. ISABELLE Parameter List

\section{Energy}

Maximum energy

Equivalent accelerator energy

Energy range, center-of-mass

$$
\begin{array}{r}
400 \times 400 \mathrm{GeV} \\
340,000 \mathrm{GeV} \\
60-800 \mathrm{GeV}
\end{array}
$$

\section{Luminosity}

Standard insertion, $400 \mathrm{GeV}$

Standard insertion, $30 \mathrm{GeV}$

Low-beta insertion, $400 \mathrm{GeV}$

High luminosity insertion, $400 \mathrm{GeV}$
$2.3 \times 10^{32} \mathrm{~cm}^{-2} \mathrm{sec}^{-1}$

$0.6 \times 10^{32} \mathrm{~cm}^{-2} \mathrm{sec}^{-1}$

$4.5 \times 10^{32} \mathrm{~cm}^{-2} \mathrm{sec}^{-1}$

$1 \times 10^{33} \mathrm{~cm}^{-2} \mathrm{sec}^{-1}$

\section{Lattice}

Circumference $\left(43 / 4 \times \mathrm{C}_{A G S}\right)$

$3834 \mathrm{~m}$

Insertion length

$6 \times 164.3 \mathrm{~m}$

Regular cell length

$54 \times 39.5 \mathrm{~m}$

Matching section length

$12 \times 59.6 \mathrm{~m}$

Horizontal separation of orhits

$94 \mathrm{~cm}$

Distance between magnets $D-D$

$0.90 \mathrm{~m}$

Distance between magnets $D-Q$

$1.00 \mathrm{~m}$

Tune $\left(\nu_{h}=\nu_{v}\right)$

22.6

Transition energy $\left(\gamma_{t r}\right)$

19.1

Amplitude function cell, $\beta_{\max }$

$67.2 \mathrm{~m}$

aximum dispersion, $X_{p}$

$2.64 \mathrm{~m}$

hase advance per cell

$\sim 90 \mathrm{deg}$

Uncorrected chromaticity, standard ring

- 33

Operating chromaticity 


\section{Standard Experimental Insertions}

$\beta_{\mathrm{v}}^{*}$ (Low-beta insertion)

$\beta_{h}^{*}$

Maximum $\beta$ (Low-beta insertion)

Total free space around crossing point

Crossing angle
7.5 (2) $\mathrm{m}$

$30 \mathrm{~m}$

153 (550) m

$60 \mathrm{~m}$

$9.4 \mathrm{mrad}$

\section{Magnet System}

Bending field at $400 \mathrm{GeV}$

$49.8 \mathrm{kG}$

at $29.4 \mathrm{GeV}$

Operating temperature

: Number of dipoles (2 rings)

Regular dipole length effective

laminations

Dipole current a $50 \mathrm{kG}$

Stored energy @ $50 \mathrm{kG} / \mathrm{dipole}$

Vacuum chamber aperture (warm bore)

Main coil i.d.

Number of quadrupoles (2 rings)

Regular quadrupole gradient

Stored energy/quadrupole

Quadrupole length effective

laminarions
$3.7 \mathrm{kr}$

$3.8 \mathrm{~K}$

732

$4.63 \mathrm{~m}$

$4.75 \mathrm{~m}$

$\sim 4 \mathrm{kA}$

$\sim 1 \mathrm{MJ}$

$8.8 \mathrm{~cm}$

$13.1 \mathrm{~cm}$

352

$6.1 \mathrm{kG} / \mathrm{cm}$

$100 \mathrm{~kJ}$

$1.65 \mathrm{~m}$

$1 . \dot{6} \dot{9} \mathrm{~m}$

\section{Cryogenic System}

Estimated heat load, $3.8 \mathrm{~K}$

Refrigeration capacity, $3.8 \mathrm{~K}$

Refrigeration capacity, $55 \mathrm{~K}$

Power requirement of compressors

Cooldown time

Cooldown weight
$13 \mathrm{~kW}$

$19 \mathrm{~kW}$

$35 \mathrm{~kW}$

$16 \mathrm{MW}$

15 days

$5 \times 10^{6} \mathrm{~kg}$ 


\section{Injection}

AGS energy

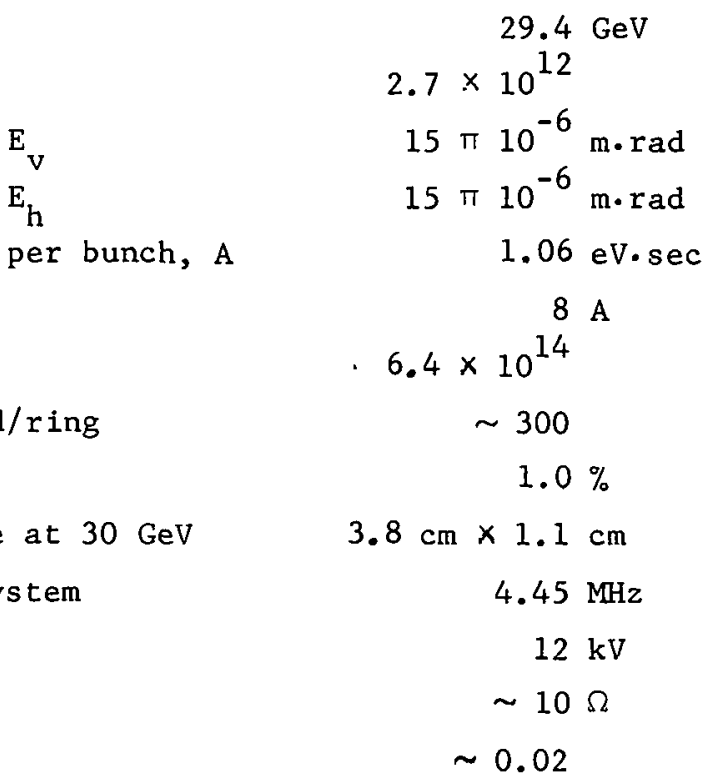

Acceleration

Duration

$\sim 4 \min$

rf frequency $(h=3)$

$235 \mathrm{kHz}$

Energy gain/turn

0.7 to $25.6 \mathrm{keV}$

Peak rf voltage/ring

$4 \times 12 \mathrm{kV}$

Total rf power/ring

$2 \mathrm{MW}$

Maximum momentum spread at $30 \mathrm{GeV}$, bunched

$1.9 \%$

Momentum spread at $400 \mathrm{GeV}$

$\sim 0.1 \%$ 


\section{REFERENCES}

Abarbane1, H.D.I. and J.B. Bronzan (1974) Phys. Rev. D9, 2367. Abarbane1, H.D.I., G.C. Chew, M.L. Goldberger, and L.M. Saunders

(1971) Phys. Rev. Lett. 26, 937.

Abers, E.S. and B.W. Lee (1973) Physics Reports 9C, No. 1. Abramovskii, V.A., O.V. Kancheli, and V.N. Gribov (1972) XVIth

Intern. Conf. on High Energy Physics, Fermilab 1, 389, and (1974) Sov. J. Nuc1. Phys. 18, 308.

ACHGT Collaboration: G. Barbiellini, M. Bozzo, P. Darriulat,

G. Diambrini Palazzi, G. DeZorzi, A. Fainberg, M.I. Ferrero,

M. Holder, A. McFarland, G. Maderni, S. Orito, J. Pilcher,

C. Rubbia, A. Santroni, G. Sette, A. Staude, P. Strolin, and

K. Tittel (1972) Phys. Lett. 39B, 663.

Amaldi, U., M. Jacob, and G. Mathias (1976) Anu. Rev. Nucl.

Sci. 26, 385 .

Ammann \& Whitney (1977) ISABELLE A Proton-Proton Colliding Beam

Facility $400 \times 400 \mathrm{GeV}$ Conventional Facilities Design Final

Report Pre-Title $I$.

Anderson, K.J., G.G. Henry, K.T. McDona1d, J.E. Pilcher,

E.I. Rosenberg, J.G. Branson, G.H. Saunders, A.J.S. Smith, and J.J. Thaler (1976) Phys. Rev, Lețters 36. 237: XVILT Tnternational Conf. on High Energy Physics, Tbilisi, USSR, 1976. Antipov, Yu. M., V.A. Bessubov, N.P. Budanov, Yu. B. Bushnin, S.P. Denisov, Yu. P. Gorin, A.A. Lebedev, A.A. Lednev,

Yu. M. Mikhailov, A.I. Petrukhin, S.A. Polovnikov,

V.A. Roinishvili, V.S. Selesnev, V.I. Sergienko, D.A. Stoyanov A.N. Sytin, Ya. A. Vazdik, and F.A. Yotch (1976) Phys. Letters 60B, 309 .

Aubert, J.J., U. Becker, J.P. Biggs, J. Burger, M. Chen,

G. Everhart, P. Goldhagen, J. Leong, T. McCorriston,

T.G. Rhoades, M. Rohde, S.C.C. Ting, S.L. Wu and Y.Y. Lee,

(1974) Phys. Rev. Letters 33, 1404. 
Augustin, J.-E., A.M. Boyarski, M. Breidenbach, F. Bulos, J.T. Dakin, G.J. Feldman, G.E. Fischer, D. Fryberger,

G. Hanson, B. Jean-Marie, R.R. Larsen, V. Luth, H.L. Lynch,

D. Lyon, C.C. Morehouse, J.M. Paterson, M.L. Perl, B. Richter,

P. Rapidis, R.F. Schwitters, W.M. Tanenbaum, F. Vannucci,

G.S. Abrams, D. Briggs, W. Chinowsky, C.E. Friedberg,

G. Goldhaber, R.J. Hollebeek, J.A. Kadyk, B. Lulu, F. Pierre, G.H. Trilling, J.S. Whitaker, J. Wiss, and J.E. Zipse (1974)

Phys. Rev. Letters 33, 1406.

Autin, B. and A.A. Garren (1975) CERN Rept. CERN/ISR-GS-MA/75-32. Barashenko, V.S. and V.I. Moskalev (1961) Fortschr, Phys. 9, 549. Barnett, R.M. (1976) in "Particles \& Fields 76," Proc. of the APS/DPF Cong., Brookhaven, D77.

Bartenev, V., A. Kuznetsov, B. Marozov, V. Nilitin, Y. Pilipenko,

V. Popov, L. Zolin, R.A. Carrigan, E. Malamud, R. Yamada,

R.L. Cool, K. Goulianos, I.H. Chiang, A.C. Melissinos,

D. Gross, and S.L. O1sen (1973a) Phys. Rev. Lett. 31, 1088. Bartenev, V., R.A. Carrigan, I-H Chiang, R.I. Cool, K. Goulianos,

D. Gross, A. Kuznetsov, E. Malamud, A.C. Melissinos,

B. Morozov, V. Nikitin, S.L. Olsen, Y. Pilipenko, V. Popov,

R. Yamada, and L. Zolin (1973b) Phys. Rev. Lett. 1ㅣ,

Berman, S.M., J.D. Bjorken, and J.B. Kogut (1971) Phys. Rev. D4, 3388 .

Beznogikh, G.G., A. Bujak, L.F. Kirillove, B.A. Morozov,

V.A. Nikitin, P.V. Nomokonov, A. Sandacz, M.G. Shafranova,

V.A. Sviridov, T. Bien, V.I. Zayachki, N.K. Zhidkov, and

L.S. Zolin (1972) Phys. Lett. $\underline{39 B}, 411$.

Beznogikh, G.G., A. Bujak, V.A. Nikitin, M.G. Shafranova,

V.A. Sviridov, T. Bien, L.V. Vikhlyantseva, V.I. Zayachki, and L.S. Zolin (1973) Phys. Lett. 43B, 85.

Binkley, M., I. Gaines, J. Peoples, B. Knapp, W. Lee, P. Leung, S.D. Smith, A. Wijangco, J. Knauer, J. Bronstein, R. Coleman. 
G. Gladding, M. Goodman, M. Gormley, R. Messner, T. O'H\&lloran,

J. Sarracino, and A. Watte (1976) Phys. Rev. Lett. 37, 571. Bjorken, J.D. (1973) Phys. Rev. D8, 4098.

BNL Schedule 44 (July 1977) Construction Project Data Sheet,

Intersecting Storage Accelerator (ISABELLE) $400 \times 400 \mathrm{GeV}$. Borer, J.P., Bramham, H. Hereward, K. Hübner, W. Schne11,

L。 Thorndah1 (1974) Proc. IXth Intern. Conf. High Energy Accelerators, Stanford, 53.

Botke, J.C. (1973) Phys. Rev. Lett. $\underline{31}, 658$.

British-Annecy-MIT-Scandanavian Collaboration (1977) presented

by N. McCubbin at ISR Discussion Meeting No, 21, editted by M. Jacob.

British-Scandinavian-MIT Collaboration: K. Guettler, B.G. Duff,

M.N. Prentice, S.J. Sharrock, W.M. Gibson, A. Duane, L. Leistam,

H. Newman, H. Ogren, H. Boggild, S. Henning, G. Jarlskog,

U. Becker, R. Little, T. Sanford, and S.L. Wu (1976) Phys.

Lett. 64B, 111 .

Brower, R.C. and J.H. Weis (1972) Phys. Lett. 41B, 631.

Brown, D.P. (1976) BNL Report ISA 76-8.

Bryant, P.J. and J.P. Gourber (1974) Proc. IXth Intern. Conf. on

High Energy Accelerators, Stanford, 87.

Bryant, P., K. Hübner, K. Johnsen, H. Laeger, B. Montague, D. Neet,

F.W. Schneider, and S. Turner (1973) IEEE Trans, Nuc1, Sci.

NS-20, No. 3, 799 .

Bugg, D.V., D.C. Salter, G.H. Stafford, K.F. George, K.F. Riley, and R.J. Tapper (1966) Phys. Rev. 146, 980 .

Burnstein, R., W.C. Carithers, M. Duong-van, R. Imlay, M. Kreisler,

V. Nauenberg, C. Rubbia, G. Snow, L. Sulak, H.H. Williams.

E. Paschos, M. Sakitt, C.L. Wang, and L.I. Wang (1975) Proc.

ISABELLE Summer study, Brookhaven, $y$.

Büsser, F.W., L. Camilleri, L. Dilella, G. Gladding, A. Placci,

B.G. Pope, A.M. Smith, J.K. Yoh, E. Zavattini, B.J. Blumenfeld,

L.M. Lederman, R.L. Cool, L. Litt, and S.L. Segler (1973)

Phys. Lett. 46B, 471; (1974) Phys. Lett. 53B, 212. 
Büsser, F.W., L. Camilleri, L. Dile11a, B.G. Pope, A.M. Smith, B.J. Blumenfeld, S.N. White, A.F. Rothenberg, S.L. Segler, M.J. Tannenbaum, M. Banner, J.B. Chèze, J.L. Hamel, H. Kasha, J.P. Pansart, G. Smadja, J. Teiger, H. Zaccone, and

A. Zylberstejn (1975) Phys. Letters 56B, 482.

Carrol1, A.S., I-H Chiang, T.F. Kycia, K.K. Li, P.O. Mazur,

P. Mockett, D.C. Rahm, R. Rubinstein, W.F. Baker, D.P. Eartly, G. Giacomel1i, P.F.M. Koehler, K.P. Pretz1, A.A. Wehmann,

R.L. Cool, and O. Fackler (1974) Phys. Rev. Lett. 33, 928. CERN-College de France-Heidelberg-Karlsruhe Collaboration:

M. Della Negra, D. Drijard, H.G. Fischer, G. Fontaine,

H. Frehse, P. Frenkiel, C. Ghesquière, R. Golieli, P. Hanke,

W. Hofmann, P.G. Innocenti, W. Isenbeck, E.E. Kluge, V. Korbel,

D. Linglin, A. Minten, A. Norton, A. Putzer, G. Sajot,

R. Sosnowski, S. Stein, J. Stiewe, H.D. Wahl, and D. Wegener,

(1977) CERN/EP/PHYS 77-10 Rev.

CERN-Rome Collaboration: U. Amaldi, G. Cocconi, A.N. Diddens,

R.W. Dobinson, J. Dorenbosch, W. Duinker, D. Gustavson,

J. Meyer, K. Potter, A.M. Wethere11, A. Baronce11i, and

C. Bosio (1976) CERN Preprint.

Chang, C-Y, E. Engels, M. Kramer, R. Lanou, L. Pondrom (1975)

Proc. ISABELLE Summer Study, Brookhaven, 224.

Chang, C-Y, G. Yodh, D. Cutts, R. Lanou, E. Engels, M. Kramer,

G. Danby, Proc. 1977 ISABELLE workshop, BNL 50721, p. 309. Chao, A.W., J.C. Herrera, and M. Month (1975), Proc. ISABELLE

Summer Study, Brookhaven, 418.

Chasman, R.W., E.D. Courant, and M. Month (1975) IEEE Trans. Nuc1.

Sci. NS-22, No. 3, 1429 .

'hasman, R. and M. Month (1976) BNL Report ISA 76-10.

Cheng, H. and T.T. Wu (1970) Phys. Rev. Lett. 24, 1456.

Cheng, H. and T.T. Wu (1973) Phys. Lett. 45B, 367.

Chicago-Princeton Collaboration: D. Antreasyan, J.W. Cronin,

H,J. Frisch, M.J. Shochet, L. Kluberg, P.A. Piroué, and 
R.L. Summer (1977) Phys. Rev. Lett. 38, 112, 115. CHOV Collaboration: H. DeKerret, E. Nagy, M. Regler, W. SchmidtParzefa11, K.R. Schubert, K. Winter, A. Brandt, F.W. Biisser, H. Dibon, G. Flügge, F. Nieberga11, P.E. Schumacher, J.J. Aubert, C。Bro11, G. Coignet, J. Favier, L. Massonnet, M. Vivargent, W. Bart1, H. Eichinger, Ch. Gottfried, and

G. Neuhofer (1976a) Phys. Lett. 62B, 363.

CHOV Collaboration: H. DeKerret, E. Nagy, M. Regler, W. SchmidtParzefa11, K.R. Schubert, K. Winter. A. Brandt, H. Dibon, G. Flïgge, F. Nieberga11, P.E. Schumacher, J.J. Aubert, C. Brol1, G. Coignet, J. Favier, L. Massonnet, M. Vivargent, W. Bart1, H. Eichinger, Ch. Gottfried, and G. Neuhofer (1976b) Phys. Lett. 63B, 477.

Chu, S-Y, B.R. Desai, B.C. Shen, and R.D. Field (1976) Phys. Rev. D13, 2967.

Chung, S.U., P. Grannis, and D. Green (1975) Proc. ISABELLE Summer Study, Brookhaven, 183.

Cobb, J.H., S. Iwata, R.B. Palmer, D.C. Rahm, I. Stumer,

C.W. Fabjan, I. Munnelli, K. Nakamura, A. Nappi, W. Struzinski,

W.J. Willis, M. Goldberg, N. Horwitz, G.C. Moneti,

C. Kourkoumelis, A.J. Lankford, and P. Rehak (1977) Phys.

Leteters 68B. 101.

Collins, P.D.B. (1971) Phys. Reports 1C, 104. CPRS Collaboration: U. Amaldi, G. Cocconi, A.N. Diddens, Z. Dimkovski, R.W. Dobinson, J. Dorenbosch, P. Duinker, G. Matthias, A.M. Thorndike, A.M. Wethere11, G. Bellettini, P.L. Braccini, R. Castaldi, V. Cavasinni, F, Cerve11i, T. Del Prete, P. Laurelli, M.M. Massa1, M. Morganti,

G. Sanguinetti, M. Voldata-Nappi, A. Baronce11i, C. Boscio, G. Abshire, J. Crouch, G. Finocchiaro, P. Grannis, H. Jöstlein, R. Kephart, D. Lloyd-Owen, and R. Thun (1976) Phys. Lett. $62 \mathrm{~B}, 460$.

Crowley-Milling, M.C. (1975) CERN Report 75-20. 
Crozier, P.J. and B.R. Webber (1976) Nucl. Phys. B115, 509. Daniels, R.E., R.W. Goodwin, M.R. Storm (1973) IEEE Trans. Nucl. Sci. NS-20, No. 3, 505 . Denisov, S.P., S.V. Donskov, Yu. P. Gorin, A.I. Petrukhin, D.A. Stoyanova, J.V. Allaby, and G. Giacome11i (1971) Phys. Lett. $36 \mathrm{~B}, 415$.

Dias de Deus, J. (1973) Nuc1. Phys. B59, 231.

Diddens, A.N. (1974) XVIIth Intern. Conf. on High Energy Physics,

London, I-41.

Jimmler, D.G. (1977) Report BNL 22465.

Donald, M.H.R., M.R. Harold,.J. Maidment, and H.G. Rees (1975)

IEEE Trans. Nuc1. Sci. NS-22, No. 3, 1433.

Dre11, S.D. and T-M Yan (1971) Ann. Phys. 66, 578. Dzhelepov, V.P., V.I. Moskalev, and S.V. Medved (1955) Sov. Phys. Daklady 104, 380 .

Edelstein, R.M. and M. Zeller (1975) Proc. ISABELLE Summer Study, Brookhaven, 234.

Edwards, D., S.0. Ohnuma, and L.C. Tend (1975a) IEEE Trans. Nuc1. Sci. NS-22, No. 3, 1426.

Edwards, D. (1975b) Proc. ISABELLE Summer Study, Brookhaven, 382. Edwards, D. (1976) Proc. ISABELLE Workshops, Brookhaven, 239. Eichten, E. and K. Gottfried (1977) Phys. Letters 66B, 286. E11is, S., M. Gaillard, D. Nanopolous (1976) Nuc1. Phys. B106, 292. Feynman, R. and M. Gell-Mann (1958) Phys. Rev. 109, 193. Field, R. and R. Feynman (1976) Cal. Tech. Report CALT-68-565. Field, R.D. and G.C. Cox (1974) Nucl. Phys. B80, 367. Fischer, J., S。 Iwata, V. Radeka, C.L. Wang, and W.J. Willis (1975) Nuc1. Instrum. Methods 127, 527.

Fischer, E. (1972) Proc. A11-Union Conf. Charged Particle Accelerators, Moscow 1 , 101 .

Foley, K.J., R.S. Jones, S.J. Lindenbaum, W.A. Love, S. Ozaki, E.D. Platner, C.A. Quarles, and E.H. Willen (1967) Phys. Rev. Lett. $19,857$. 
Foley, K.J. and B.T. Meadows (1975) Proc. ISABELLE Summer Study, Brookhaven, 292.

Fox. G. (1976) in "Particles \& Fields 76," Proc. of the APS/DPF Conf., Brookhaven, Gl.

Frazer, W.R. and D.R. Snider (1973) Phys. Lett. 45B, 136.

Frisch, H。 (1976) in "Particles \& Fields 76," Proc. of the APS/DPF

Conf., Brookhaven, F59.

Gaisser, T.K. and C.I. Tan (1973) Phys. Rev. D8, 3881.

Gaisser, T.K. and F. Halzen (1975) Phys. Rev. D11, 3157.

Gaisser, T.K., F. Ha1zen, and E.A. Paschos (1977) Phys. Rev. D1.5, 2572.

Gareyte, J. and J.P. Gourber (1975) Proc. ISABELLE Summer Study,

Brookhaven, 235.

Garren, A. (1975) Proc. ISABELLE Summer Study, Brookhaven, 814. Gel1-Mann, M. (1959) Rev. Mod. Phys. 31, 834.

Giacome11i, G. and A. Thorndike (1975) Proc. ISABELLE Summer

Study, Brookhaven, 301.

Glordano, S。 (1975) Proc. ISABELLE Summer Study, Brookhaven, 508. Glashow, S. (1975) Sci. Am. 233, No. 4, 38.

Grand, P. (1972) BNL Report CRISP 72-18.

Gröbner, 0. and P. Strubin (1977) private communication. Gröbner, O. and P. Strubin (1976) private communication. Grunder, H.A. and G.R. Lambertson (1971) Proc. VIIIth Intern.

Conf. High Energy Accelerators, CERN, 291. Gunion, J.F., S.J. Brodsky, and R. Blankenbecler (1972) Phys. Rev. D6, 2652 .

Hahn, H., M. Month, and R.R. Rau (1977), Kev. Mod. Phys. 49, 625. Halama, H.J. and J.C. Herrera (1975) IEEE Trans. Nuc1 Sci. NS-22, No. 3, 1492.

Halama, H.J. (1977) J. Vac。Sci. Technol. (in press). Hansen, S. and M.J. DeJonge (1971) Proc.VIIIth Intern. Conf.

High Energy Accelerators, CERN, 505. Harting, D., P. Blacka11, B. Elsner, A.C. He1mholtz, 
W.C. Middlekoop, B. Powe11, B. Zacharov, P. Zanella, P. Dalpiaz, M.N. Focacci, S. Focardi, G. Giacomelli, L. Monari, J.A. Beaney, R.A. Donald, P. Mason, L.W. Jones, and D.0. Caldwel1 (1965) Nuovo Cimento 38,60 。

Hauviller, C., H. Schonbacher, and A. van Steenbergen (1977)

"Beam Dump Absorber for the Superconducting ISR" CERN Report, in preparation.

Hendrik, R.E., PI Langacker, B.E. Lautrup, S.J. Orfanidis, and

V. Rittenberg (1975) Phys. Rev. D11, 536.

IEPAP (1977) Report of the 1977 Subpanel on New Facilities of the

High Energy Physics Advisory Panel, ERDA 77-71.

Hendrickson, K.N. and M.J. DeJonge (1974) Proc. IXth Intern.

Conf. on High Energy Accelerators, 390.

Herb, S.W., D.C. Hom, L.M. Lederman, J.C. Sens, H.D. Snyder, J.K. Yoh, J.A. Appe1, B.C. Brown, C.N. Brown, W.R. Innes, K. Ueno, T. Yamanouchi, A.S. Ito, H. Jöstlein, D.M. Kaplan, and R.D. Kephart (1977) Phys. Rev. Letters 39, 252.

Hereward, H.G. (1975) Proc. ISABELLE Summer Study, Brookhaven, 555. Hereward, H.G. (1971) CERN Report, 71-15. Herrera, J.C. (1976a) BNL Formal Report ISA 76-12. Herrera, J.C. (1976b) Proc. ISABELLE Workshops, Brookhaven, 236. Hom, D.C., L.M. Lederman, H.P. Paar, H.D. Snyder, J.M. Weiss, J.K. Yoh, J.A. Appel, B.C. Brown, C.N. Brown, W.R. Innes, T. Yamanouchi, R.J. Englemann, R.J. Fisk, H. Jöstileiı, D.M. Kaplan, R.D. Kephart, and R.L. McCarthy (1976) Phys. Rev. Lett. $\underline{37}, 1374$.

Imlay, R., S. Iwata, S. Jacobs, R. Kraemer, M. Kreisler,

K. McDonald, P. McIntyre, J. Thompson, A. Walenta, I.H. Chiang, M. Sakitt, A. Thorndike, C. Wang, and L.L. Wang (1976) Proc. ISABELLE Workshops, Brookhaven, 24.

ISA (1972-76) "200 GeV Intersecting Storage Accelerators, ISABELLE" (1972) BNL 16716, "A Proposal for Construction of a ProtonProton Storage Accelerator Facility - ISABELLE," (1974) BNL 18891, BNL 19273, (1975) BNL 20161, (1976) BNL 50519, (1977) BNL 50648 . 
Kei1, E. and B。Zotter (1971) CERN Report, ISR-TH/71-58.

Kluberg, L., P.A. Piroué, R.I. Sumner, D. Antreasyan, J.W. Cronin, H.J. Frisch, and M.J. Schochet (1976) Phys. Rev. Lett. 37, 1451. Lederman, L.M. and B.G. Pope (1976) "Dilepton Production and

Scaling", Columbia/CERN preprint.

Lederman, L.M. (1977) Proc. Intern. Symp. on Lepton and Photon

Interactions at High Energies, Hamburg.

Lillethun, E. (1973) Acta Phys. Pol. B4, 769.

Limon, P. and R. Majka (1976) Proc. ISABELLE Workshops, Brookhaven, 188 .

Linde, A.D. (1976) JETP Letters 23, 73.

Ma11ory, K.B. (1975a) Proc. ISABELLE Summer Study, Brookhaven, 160. Ma11ory, K.B. (1975b) IEEE Trans. Nuc1. Sci. NS-22, No. 3, 1086. Marx, J.N. (1974) BNL Report, CRISP 74-3.

Marx, J.N. and R.D. Majka (1975) Proc. ISABELLE Summer Study, Brookhaven, 204 .

McDanie1, B.D. (1975) "A Proposal to the NSF for Construction Funds to Modify the Cornel1 Electron Synchrotron Facility to Provide an Electron-Positron Colliding Beam Facility," 75-78. McDona1d, K.T. and J.J. Thaler (1977) Proc. ISABELLE Workshops, BNL 50721, 160 .

McInturff, A.D., W.B. Sampson, K.E. Robins, P.F. Dahl, and

R. Damm (1977) IEEE Trans. Nuc1. Scị. NS-24, 1242 . Migda1, A.A., A.M. Polyakox, and K.A. Ter-Martirosyan (1974)

Zh. Eksp. Teor. Fiz. 67, 2009.

Month, M. (1972) Particle Accelerators 3, 183; A.A. Garren (1975)

Proc. ISABELLE Summer Study, Brookhaven, 372.

Month, M. and K. Je11ett (1973) Nucl. Instrum. Methods 113.

453; B。Zotter (1972) CERN Report CERN-ISR-TH 172-36. Mueller, A.H. (1970) Phys. Rev. D2, 2963.

Mueller, A.H. (1971) Phys. Rev. D4, 150. Nambu, Y. (1976) Sci. Am. 235, No. 5, 48. Palmer, R. (1977) Brookhaven CERN Syracuse Yale Collaboration, preliminary results private communication. 
Palmer, R.B. (1975) BNL Report, CRISP 75-4.

Palmer, R.B., E.A. Paschos, N.P. Samios and L-L Wang (1976)

Phys. Rev. D14, 118 。

Parzen, G. (1975a) Proc. ISABELLE Summer Study, Brookhaven, 573.

Parzen, G. (1975b) Particle Accelerators 6, 237.

Pavia-Princeton Collaboration: M. Cavalli-Sforza, C. Conta,

M. Fraternali, G. Goggi, F. Impellizzeri, G.C. Mantovani,

F. Pastore, S. Ratti, B. Rossini, D. Scannicchio, and

H. Sadrozinski (1975) Lettere al Nuovo Cimento 14, 345.

?eier1s, R., T.L. Trueman, and L.L. Wang (1977) Phys. Rev. D16, 1397.

Peierls, R. (1975) Proc. ISABELLE Summer Study, Brookhaven, 327.

Peoples, J. (1976) Proc. ISABELLE Workshops, Brookhaven, 199.

Joint Lawrence Berkeley Laboratory and Stanford Linear Accelerator

Center (1974) "A Proposal for a Positron-Electron Colliding

Beam Storage Ring Project (PEP)," LBL2688/SLAC-171, 77-80.

Peterson, J., A. van Steenbergen, and L.C. Teng (1975) Proc.

ISABELLE Summer Study, Brookhaven, 447.

Pisa-Stony Brook Collaboration: S.R. Amendilia, G. Bellettini,

P.L. Braccini, C. Bradaschia, R. Castaldi, V. Cavasinni,

C. Cerri, T. Del Prete. L. Foa, P. Giromini, P. Laurel1i,

A. Menzione, L. Ristori, G. Sanguinetti, M. Valdata,

G. Finocchiaro, P. Grannis, D. Green, R. Kephart, and R. Thun

(1974) Phys. Lett. 48B, 359.

Quigg, C. (1973) Experiments on High Energy Particle Collisions,

Vanderbilt, 375 .

Resegotti, L. and B. Montague (1976) private communication.

icherer, F. (1973) IEEE Trans. Nuc1. Sci. NS-20, No. 3, 825.

Sacherer, F. (1974) Proc. IXth Intern. Conf. High Energy

Accelerators, Stanford, 347.

Salam, A. (1969) in "Elementary Particle Theory," edited by

N. Svartholm, (Almquist and Wikse11, Stockho1m), p. 367.

Sampson, W.B., K.E. Robins, S. Kiss, P.F. Dah1, and A.D. McInturff

(1977) Trans. IEEE Nucl. Sci. NS-24, 1296. 
Schne11, W. (1975a) Proc. ISABELLE Summer Study, Brookhaven, 126. Schne11, W. (1975b) IEEE Trans. Nuc1. Sci. NS-22, No. 3, 1358. Schwaller, P., M. Pepin, D.F. Meadsday, P.-U Renberg, B. Favier, and C. Richard-Serre (1971) Phys. Lett. 35B, 243. Shutt, R.P., (1976) BNL Report, ISA Tech Note No. 8. Sivers, D., S. Brodsky, R. Blankenbecler (1976) Phys. Repts. 23C. Smith, L. (1975) Proc. ISABELLE Summer Study, Brookhaven, 111. Snyder, H.D., D.C. Hom, L.M. Lederman, H.P. Paar, J.M. Weiss, J.K. Yoh, J.A. Appel, B.C. Brown, C.N. Brown, W.R. Innes, T. Yamanouchi, and D.M. Kaplan (1976) Phys. Rev. Letters 36,1415 .

van Steenbergen, A. (1975) BNL Report ISA 75-6. Vosicki, B. and K. Zankel (1975) J. Phys. E8, 1 . Webb, R., G. Trilling, V. Telegdi, P. Strolin, B. Shen, P. Schlein, J. Rander, B. Naroska, T. Meyer, W. Marsh, W. Lockman, J. Layter, A. Kernan, M. Hansroul, S-Y Fung, H. Foeth, R. Ellis, A. Derevshikov, M. Bozzo, A. Böhm, and L. Baksay (1975) Phys. Lett. 55B, 331. Weinberg, S. (1976) Phys. Rev. Lett. 36, 294. Weinberg, S. (1967) Phys. Rev. Lett. 19, 1264. White, D.H. (1976) Proc. ISABELLE Workshops, Brookhaven, 54. Wiedemann, H. (1976) Proc. ISABELLE Summer Workshops, Brookhaven, 227.

Williams, H. (1976) in "Particles \& Fields 76," Proc. of the APS/DPF Conf. Brookhaven, D95. Wil1is, W.J. (1976) Proc. ISABELLE Workshops, Brookhaven, 170. Yamaguchi, Y (1966) Nuovo Cimento 43, 193. Zotter, B. (1972) CERN Report CERN-ISR-TH/172-36. 